\title{
CHARACTERIZING NEUROTRANSMITTER RECEPTOR ACTIVATION WITH A PERTURBATION BASED DECOMPOSITION METHOD
}

\author{
A Thesis \\ presented to \\ the Faculty of California Polytechnic State University, \\ San Luis Obispo
}

\author{
In Partial Fulfillment \\ of the Requirements for the Degree \\ Master of Science in Biomedical Engineering
}

by

Stephen Jue

June 2016 
(C) 2016

Stephen Jue

ALL RIGHTS RESERVED 


\section{COMMITTEE MEMBERSHIP}

TITLE:

AUTHOR:

DATE SUBMITTED:

COMMITTEE CHAIR: Robert Szlavik, Ph.D.

Professor of Biomedical Engineering

COMMITTEE MEMBER: Scott Hazelwood, Ph.D.

Professor of Biomedical Engineering

COMMITTEE MEMBER: David Clague, Ph.D.

Professor of Biomedical Engineering 


\begin{abstract}
Characterizing Neurotransmitter Receptor Activation with a Perturbation Based Decomposition Method
\end{abstract}

Stephen Jue

The characterization of postsynaptic potentials, in terms of neurotransmitter receptor activation, is of clinical significance because information associated with receptor activation can be used in the diagnosis and study of neurological disorders. Single-unit recordings provide a method of measuring postsynaptic potentials in neurons using a microelectrode system, but yield no detailed information regarding the neurotransmitter receptors that contribute to the potential. To determine the types of neurotransmitter receptors that result in a compound postsynaptic potential from a microelectrode reading, decomposition of the potential is necessary. In this work, a perturbation-based decomposition method developed by R. Szlavik is evaluated for this application, and compared to a generalized Fourier series approach. The resultant estimator is valid for decomposition of multiple-receptor compound postsynaptic potentials as well as single-receptor compound postsynaptic potentials. The estimator also yields a satisfactory decomposition of experimental postsynaptic potential data found in the literature. 


\section{ACKNOWLEDGMENTS}

Thanks to:

- Dr. Szlavik, for giving me the opportunity to work on such exciting research, and for providing support and guidance

- Dr. Hazelwood and Dr. Clague for agreeing to be on my committee

- My friends and family for their support

- Andrew Guenther, for uploading this template [1] 


\section{TABLE OF CONTENTS}

Page

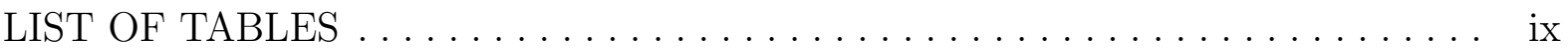

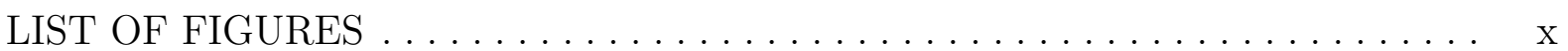

LIST OF ABBREVIATIONS ..................... xii CHAPTER

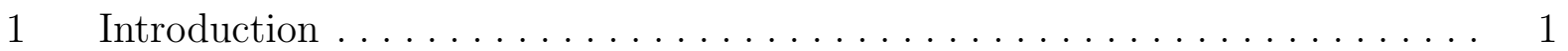

1.1 Motivation ........................... 1

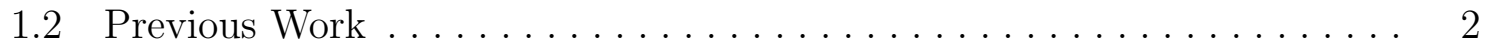

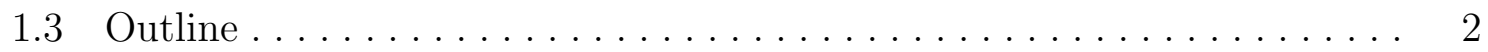

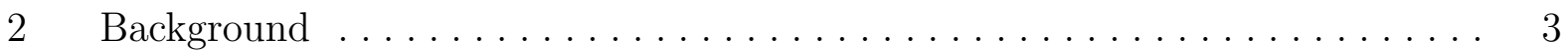

2.1 The Human Nervous System $\ldots \ldots \ldots \ldots \ldots \ldots \ldots \ldots \ldots \ldots \ldots \ldots$

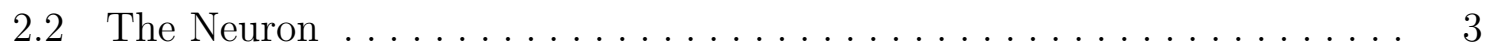

2.2 .1 Neural Signaling . . . . . . . . . . . . . . . 5

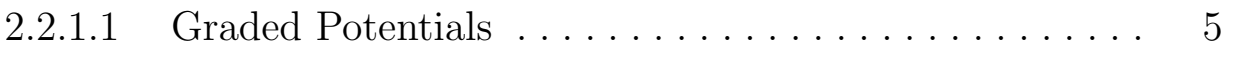

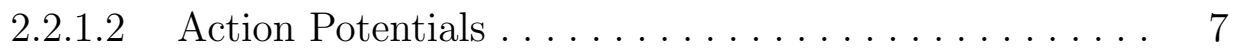

2.3 The Synapse . . . . . . . . . . . . . . . . . . . . . 8

2.3.1 Information Transfer Across Chemical Synapses . . . . . . . . . . 8

2.3.2 Neurotransmitters . . . . . . . . . . . . . . . . . . . . . . . 11

2.3.2.1 Glutamate ................... 12

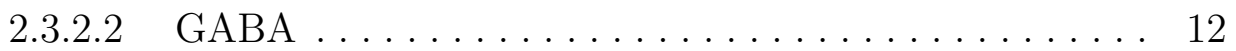

2.3.3 Neurotransmitter Receptors . . . . . . . . . . . . . . . . . . . . 12

$3 \quad$ Methods .............................. 15

3.1 Postsynaptic Potential Models . . . . . . . . . . . . . . . . . . . 15

3.1.1 Synaptic Conductance Model . . . . . . . . . . . . . . . . 16 
3.1.2 Solving for Postsynaptic Potential Numerically . . . . . . . . . . . . 18

3.2 Generalized Fourier series approach . . . . . . . . . . . . . . . . . 19

3.3 Perturbation Decomposition Method ................... 20

3.3.1 Generalizing the Perturbation Decomposition Method . . . . . . 21

3.4 Decomposition of Compound Postsynaptic Potential . . . . . . . . . . . . . . 22

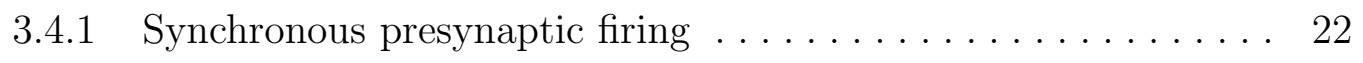

3.4 .2 Asynchronous presynaptic firing . . . . . . . . . . . . . 23

3.4.2.1 AMPA-mediated potentials . . . . . . . . . . 23

3.4.2.2 GABA $_{\mathrm{A}, \text { slow }}$ mediated potentials ........... 24

3.4.2.3 GABA $_{\mathrm{A}, \text { fast-mediated potentials } \ldots \ldots \ldots \ldots} \ldots \ldots$

3.4.2.4 NMDA-mediated potentials . . . . . . . . . . 24

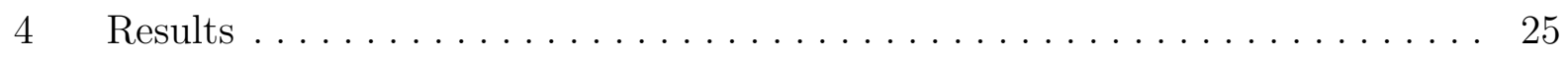

4.1 Synchronous presynaptic firing . . . . . . . . . . . . . . . 25

4.2 Asynchronous presynaptic firing . . . . . . . . . . . . . . 28

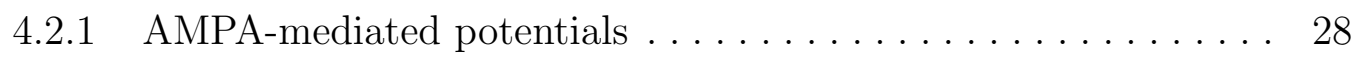

4.2.2 GABA $_{\mathrm{A}, \text { slow }}$ mediated potentials ............. 32

4.2.3 GABA $_{\mathrm{A}, \text { fast }}$-mediated potentials $\ldots \ldots \ldots \ldots \ldots$

4.2 .4 NMDA-mediated potentials . . . . . . . . . . . . . . . 40

4.3 Experimental validation $\ldots \ldots \ldots \ldots \ldots \ldots \ldots \ldots \ldots \ldots \ldots$

$5 \quad$ Discussion ............................. 49

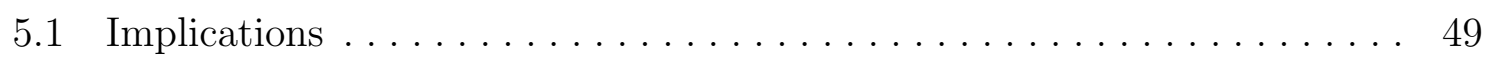

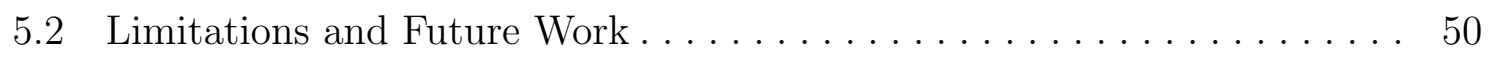

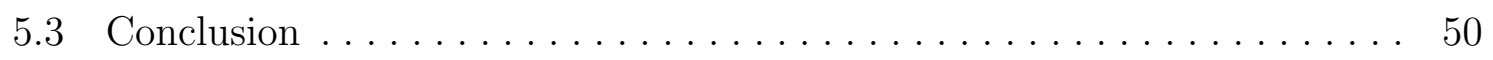

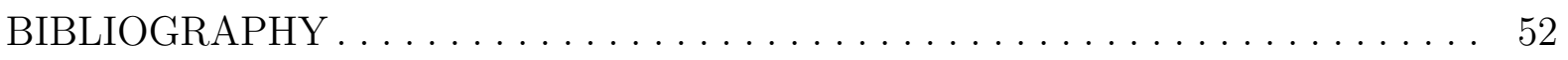

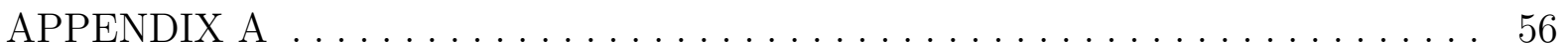




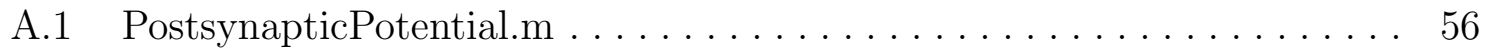

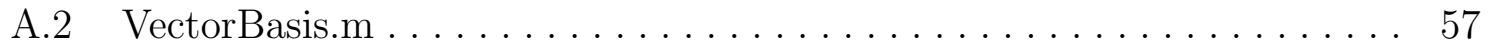

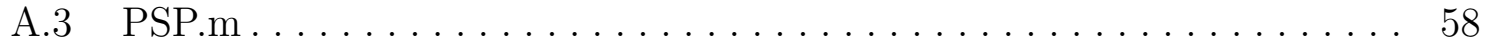

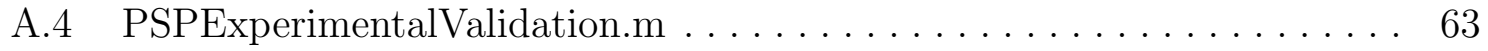




\section{LIST OF TABLES}

Table

Page

$3.1 \quad$ Values used in Postsynaptic Potential Models. . . . . . . . . . . . . . . . . 16

3.2 Values used in Synaptic Conductance Models. . . . . . . . . . . . . . . . . 17

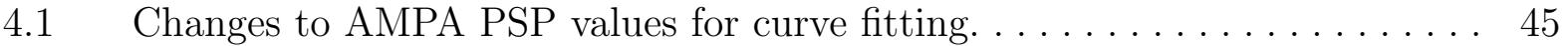




\section{LIST OF FIGURES}

Figure

Page

2.1 The human nervous system $[12] \ldots \ldots \ldots \ldots \ldots \ldots \ldots \ldots \ldots \ldots \ldots$

2.2 Structure of a motor neuron $[12] \ldots \ldots \ldots \ldots \ldots \ldots \ldots \ldots \ldots \ldots \ldots \ldots \ldots \ldots$

2.3 Excitatory and inhibitory postsynaptic potentials $[12] \ldots \ldots \ldots 6$

2.4 Different cases of IPSP and EPSP summation $[12] \ldots \ldots \ldots \ldots \ldots$

2.5 Action potential propagation along an axon $[12] \ldots \ldots \ldots \ldots \ldots$

$2.6 \quad$ Ligand-gated ion channel $[12] \ldots \ldots \ldots \ldots \ldots \ldots \ldots \ldots \ldots \ldots \ldots \ldots \ldots \ldots$

2.7 Chemical synapse signal transmission $[12] \ldots \ldots \ldots \ldots \ldots \ldots \ldots$

2.8 Structural formulas of common neurotransmitters $[13] \ldots \ldots \ldots \ldots \ldots$

2.9 Ionotropic and metabotropic neurotransmitter receptors $[14] \ldots \ldots \ldots \ldots$

2.10 GABAergic drugs as a treatment of stress-related disorders [19] . . . . . . 14

3.1 Postsynaptic Potentials due to binding to four different types of receptors: AMPA, NMDA, GABA , fast $_{\text {and }} \mathrm{GABA}_{\mathrm{A}, \text { slow }} \ldots \ldots \ldots \ldots \ldots \ldots$

4.1 Basis functions for synchronous presynaptic firing, $v_{A}(t), v_{N}(t), v_{S}(t), v_{F}(t)$. 26

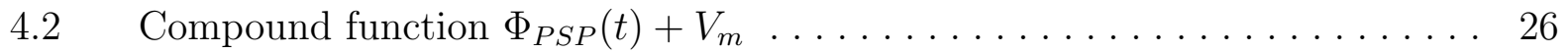

$4.3 \quad$ Frequency distribution from decomposition of $\Phi_{P S P}(t) \ldots \ldots \ldots \ldots$

4.4 Comparison of error from the generalized Fourier series approach $\left(\left|\beta_{k}-\xi_{k}^{\prime}\right|\right)$ to Szlavik perturbation decomposition approach $\left(\left|\beta_{k}-\beta_{k}^{\prime}\right|\right) \ldots \ldots \ldots \ldots \ldots$

4.5 Basis functions for asynchronous presynaptic firing of AMPA-mediated neu-

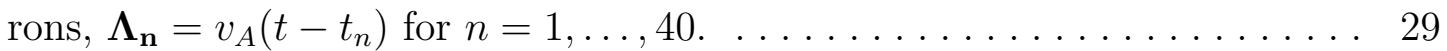

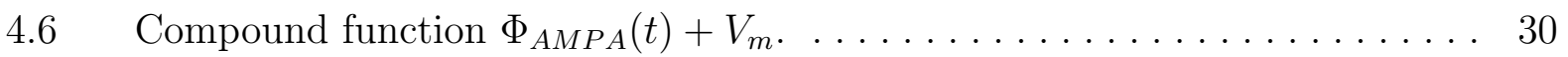

4.7 Frequency distribution from decomposition of $\Phi_{A M P A}(t) \ldots \ldots \ldots \ldots 1$

4.8 Comparison of error from generalized Fourier series approach to Szlavik perturbation decomposition approach for AMPA-mediated potentials. . . . . 32

4.9 Basis functions for asynchronous presynaptic firing of $\mathrm{GABA}_{\mathrm{A}}$, slow-mediated neurons, $\boldsymbol{\Lambda}_{\mathbf{n}}=v_{S}\left(t-t_{n}\right)$ for $n=1, \ldots, 20 \ldots \ldots \ldots \ldots \ldots \ldots \ldots$

4.10 Compound function $\Phi_{G A B A, s l o w}(t)+V_{m} \ldots \ldots \ldots \ldots \ldots \ldots$

4.11 Frequency distribution from decomposition of $\Phi_{G A B A, \text { slow }}(t) \ldots \ldots$. . . . . 35

4.12 Comparison of error from generalized Fourier series approach to Szlavik

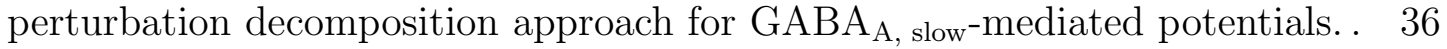


4.13 Basis functions for asynchronous presynaptic firing of $\mathrm{GABA}_{\mathrm{A}}$, fast-mediated neurons, $\boldsymbol{\Lambda}_{\mathbf{n}}=v_{F}\left(t-t_{n}\right)$ for $n=1, \ldots, 20 \ldots \ldots \ldots \ldots \ldots \ldots \ldots \ldots$

4.14 Compound function $\Phi_{G A B A, \text { fast }}(t)+V_{m} \ldots \ldots \ldots \ldots \ldots \ldots \ldots \ldots$

4.15 Frequency distribution from decomposition of $\Phi_{G A B A, f a s t}(t) \ldots \ldots \ldots \ldots$

4.16 Comparison of error from generalized Fourier series approach to Szlavik perturbation decomposition approach for $\mathrm{GABA}_{\mathrm{A}}$, fast-mediated potentials. . 40

4.17 Basis functions for asynchronous presynaptic firing of NMDA-mediated neurons, $\boldsymbol{\Lambda}_{\mathbf{n}}=v_{N}\left(t-t_{n}\right)$ for $n=1, \ldots, 20 \ldots \ldots \ldots \ldots \ldots \ldots \ldots \ldots \ldots$

4.18 Compound function $\Phi_{N M D A}(t)+V_{m} \ldots \ldots \ldots \ldots \ldots \ldots \ldots \ldots \ldots$

4.19 Frequency distribution from decomposition of $\Phi_{N M D A}(t) \ldots \ldots \ldots \ldots 4$

4.20 Comparison of error from generalized Fourier series approach to Szlavik perturbation decomposition approach for NMDA-mediated potentials..... . 44

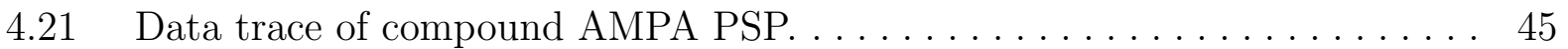

4.22 Basis functions for decomposition of traced data from Abrahamsson et al. . 46

4.23 Frequency plot of Perturbation Decomposition Method on experimental data. Dashed red line denotes chosen cutoff frequency, $f_{c}=0.3 \ldots \ldots .47$

4.24 Reconstruction of EPSP spike train from Abrahamsson et al. study [29]. . . 48

4.25 Comparison of Szlavik perturbation decomposition estimations $\left(\beta_{k}^{\prime}\right)$ to generalized Fourier series estimations $\left(\xi_{k}^{\prime}\right)$ on experimental data from Abrahamsson et al. study. . . . . . . . . . . . . . . . . . . . . . 48 


\section{LIST OF ABBREVIATIONS}

AMPA $\alpha$-amino-3-hydroxy-5-methyl-4-isoxazolepropionic acid

AP Action potential

CNS Central nervous system

EEG Electroencephalogram

EPSP Excitatory postsynaptic potential

GABA Gamma-Aminobutyric acid

IPSP Inhibitory postsynaptic potential

NMDA N-methyl-D-aspartate

PNS Peripheral nervous system

PSP Postsynaptic potential 


\section{Introduction}

The human body is an unimaginably complex system, and nowhere else is this better illustrated than in the brain. The average human brain has 100 billion neurons [2], while the cerebral cortex has about 0.15 quadrillion synapses between its neurons [3]. However, hidden in this complexity is a remarkable simplicity: the neurotransmitters mediating the communication between neurons. While there are around 50-100 known neurotransmitters [4], just a handful of them do the majority of the work. Since most activity in the brain is mediated by chemical synapses, almost all of the signals in the brain can be characterized by a few different neurotransmitter receptors. Many disorders in the brain cause changes in the activation frequency of these receptors, so being able to detect these changes would be useful. The purpose of this work is to present and evaluate a method that estimates the frequency of neurotransmitter receptor activation from a whole-cell patch-clamp voltage recording of a neuron.

\subsection{Motivation}

It is well known that many disorders, including Parkinson's disease and Alzheimer's disease, are caused, in part, by neurotransmitter receptor dysfunction [5]. In fact, it is widely believed that Alzheimer's disease begins as a malfunction of synapses. The amyloid- $\beta$ peptide, which accumulates in the brains of patients with Alzheimer's disease, has been shown to impair memory by influencing the removal of NMDA receptors [6] and $\mathrm{GABA}_{\mathrm{A}}$ receptors [7].

To facilitate diagnosis and study of such disorders, a technique to characterize neural signals by neurotransmitter receptor activation would be useful. This work will present and evaluate such a technique. In particular, a method of "decomposing" a patch-clamp recording into single-neuron postsynaptic potentials (PSPs) will be presented and evaluated. Specifically, this method will decompose a compound PSP into four components: NMDA 
receptor-mediated PSP, AMPA receptor-mediated PSP, fast-responding GABA $\mathrm{A}_{\mathrm{A}}$ receptormediated PSP, and slow-responding $\mathrm{GABA}_{\mathrm{A}}$ receptor-mediated PSP. This method can be used to estimate the frequency of activation of NMDA receptors, AMPA receptors, fastresponding $\mathrm{GABA}_{\mathrm{A}}$ receptors, and slow-responding $\mathrm{GABA}_{\mathrm{A}}$ receptors, which could be used for the diagnosis and study of neurological disorders.

\subsection{Previous Work}

The related problem of determining the time course of neurotransmitter in the synaptic cleft has spanned more than 60 years of neuroscience research, and has yet to have a definite consensus. A study by Scimemi et al. overviews some of the attempts, such as quantal analysis, mathematical modeling of synaptic currents, as well as sniffer and imaging approaches [8]. Some in vitro studies have sought to determine the contribution of AMPA receptors [9] and other classes of glutamate receptors [10] to postsynaptic potentials using pharmacological isolation techniques. Another in vitro work analyzed the contribution of AMPA, NMDA, and $\mathrm{GABA}_{\mathrm{A}}$ receptors to temporal patterns in PSPs of the inferior colliculus of the rat [11]. Though there have been many in vitro studies doing component analysis of PSPs, very little, if any, simulation-based PSP decomposition methods have been developed. This work aims to introduce this novel PSP analysis technique as a way to study neurotransmitters and their receptors.

\subsection{Outline}

The paper will be structured as follows: First, a background on the biology and biophysics of synaptic transmission will be provided. Then, R. Szlavik's perturbation decomposition method will be described and adapted to this problem with the use of biophysical models in the literature. Finally, the method will be evaluated by applying it to simulated postsynaptic

potentials and to experimental data, and comparing it to a generalized Fourier series method. 


\section{Background}

\subsection{The Human Nervous System}

The human nervous system can be divided into two branches: the central nervous system (CNS) and the peripheral nervous system (PNS). The CNS is the control center of the nervous system - it interprets sensory input and dictates output based on reflexes, past experiences, and current conditions. The PNS consists mainly of nerves (bundles of axons) and ganglia (collections of cell bodies). It can be further divided into the sensory division, which consists of neurons conveying impulses to the central nervous system from sensory receptors, and the motor division, which transmits signals from the CNS to muscles and glands.

\subsection{The Neuron}

Neurons are highly specialized cells that conduct messages in the form of nerve impulses throughout the body. They coordinate with other cells and systems to perform various functions, including information processing, muscle coordination, and sensory reception and processing. To accomplish such feats, they are arranged in complicated neural networks throughout the body and connected to sensory receptors, muscles, and other organs to relay messages to and from the brain and spinal cord.

The cell body, or soma, of a neuron ranges in diameter from 5 to $140 \mu \mathrm{m}$ [12]. The soma has dendrites, which are short, branching extensions that provide an enormous surface area for receiving signals from other neurons. The small gap between connected neurons is called the synapse. Each neuron has an axon, which is a slender process of uniform diameter. Some neurons have very short axons, while others - such as the motor neurons controlling the skeletal muscles in the feet - can extend over a meter in length. A long axon is called a nerve fiber. At the end of the axon are knoblike terminal branches, called axon terminals. 


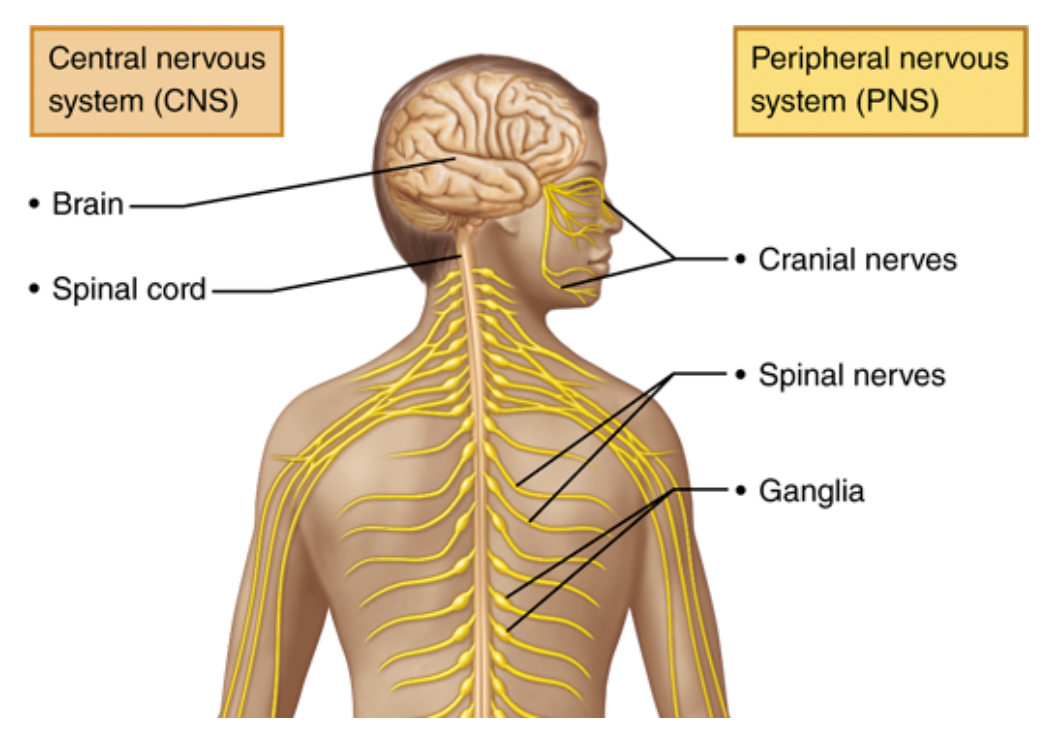

Figure 2.1: The human nervous system [12].

The axon is the conducting region of the neuron - it generates and transmits nerve impulses, or action potentials. When the action potential reaches the axon terminals, it causes neurotransmitters - signalling chemicals - to be released. These neurotransmitters either inhibit or excite other neurons.

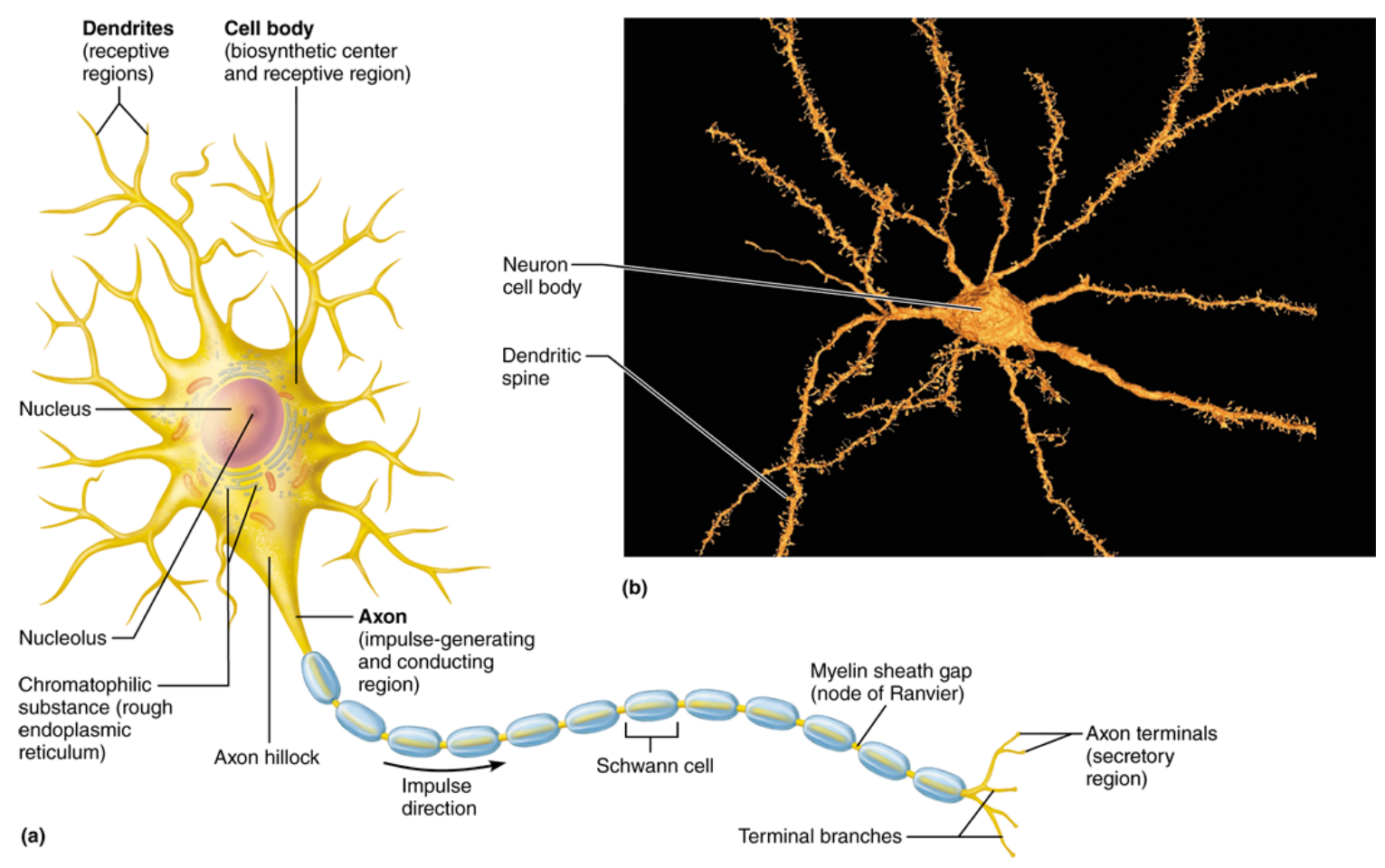

Figure 2.2: Structure of a motor neuron [12]. 


\subsubsection{Neural Signaling}

Like all cells, neurons have a resting membrane potential $\left(V_{m}\right)$. A neuron's membrane is said to be polarized, with a potential difference of -40 to $-90 \mathrm{mV}$ depending on the type of neuron [12]. This potential difference is due to the differences in $\mathrm{Na}^{+}$and $\mathrm{K}^{+}$concentrations inside and outside the cell, as well as differences in permeability of the plasma membrane to these ions. Namely, potassium ions can flow out of the neuron much more easily than sodium ions can flow in, causing a negative resting potential. To maintain this ionic imbalance (and thus maintain the resting membrane potential), neurons have sodium-potassium pumps powered by adenosine triphosphate.

Neurons receive and send information through changes in their membrane potential. These voltage changes are caused by anything that alters the membrane permeability to an ion. A positive change in voltage is called a depolarization, while a negative change is called a hyperpolarization. Changes in membrane potential can cause two types of signals: graded potentials and action potentials.

\subsubsection{Graded Potentials}

Graded potentials are short-lived, localized changes in membrane potential that typically occur in the soma or axon hillock of the neuron. When a graded potential is triggered by a neurotransmitter released by another neuron, it is called a postsynaptic potential (PSP). For a neuron to fire an action potential, the membrane potential must reach the threshold voltage $\left(V_{t}\right)$ of the neuron. This voltage is more positive than the resting membrane potential, $V_{m}$, as depicted in Figure 2.3.

There are two types of postsynaptic potentials: inhibitory postsynaptic potentials (IPSPs) and excitatory postsynaptic potentials (EPSPs). IPSPs decrease the neuron's membrane voltage and thus make it less likely to fire an action potential, while EPSPs make the membrane voltage more positive, bringing it closer to the threshold voltage and thus increasing the chance that it will fire. 
(a) Excitatory postsynaptic potential (EPSP)

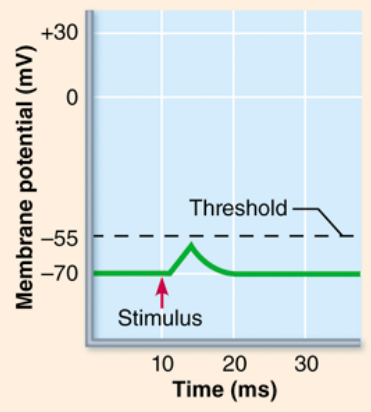

An EPSP is a local depolarization of the postsynaptic membrane.

- EPSPs bring the neuron closer to AP threshold.

- Neurotransmitter binding opens chemically gated ion channels, allowing $\mathrm{Na}^{+}$and $\mathrm{K}^{+}$to pass simultaneously. (b) Inhibitory postsynaptic potential (IPSP)

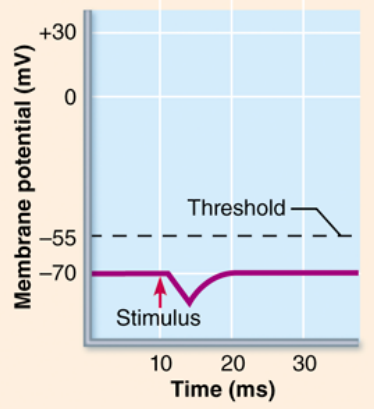

An IPSP is a local hyperpolarization of the postsynaptic membrane.

- IPSPs drive the neuron away from AP threshold.

- Neurotransmitter binding opens chemically gated ion channels permeable to either $\mathrm{K}^{+}$or $\mathrm{Cl}^{-}$.

Figure 2.3: Excitatory and inhibitory postsynaptic potentials [12].

The cumulative effect of two PSPs close in time is called temporal summation, while the cumulative effect of two simultaneous stimuli at different locations is called spatial summation. Summation can be additive (EPSP + EPSP) or subtractive (EPSP + IPSP). Figure 2.4 depicts four different cases. The inner figures (b) and (c) show the membrane potential reaching threshold and firing an action potential, while the outer figures (a) and (d) show the membrane potential staying at subthreshold levels.
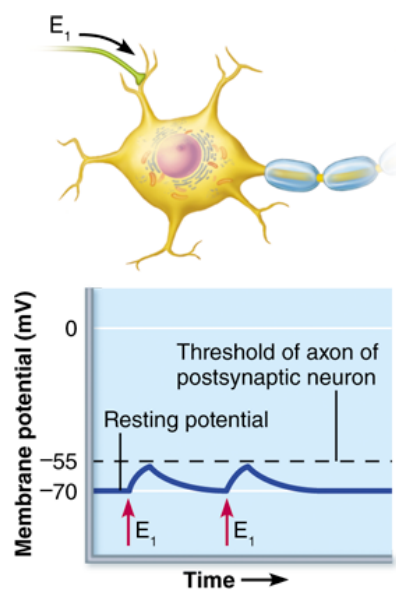

(a) No summation: 2 stimuli separated in time cause EPSPs that do not add together.
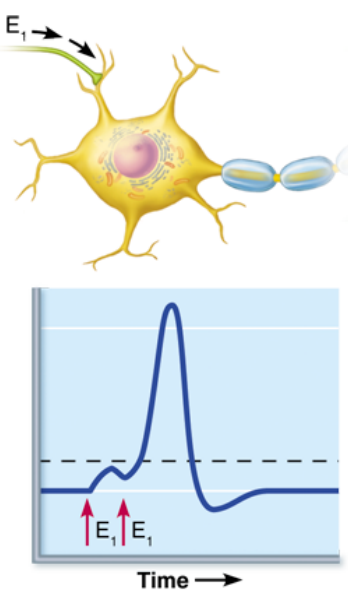

(b) Temporal summation: 2 excitatory stimuli close in time cause EPSPS that add together.
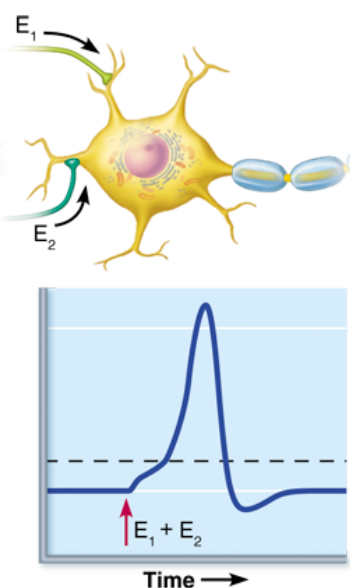

(c) Spatial summation: 2 simultaneous stimuli at different locations cause EPSPs that add together.
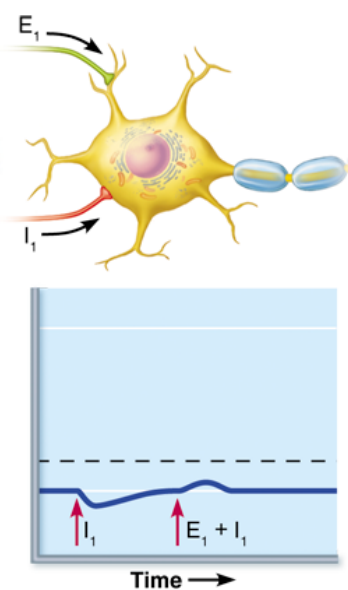

(d) Spatial summation of EPSPs and IPSPs:

Changes in membrane potential can cancel each other out.

Excitatory synapse $1\left(E_{1}\right)$

Excitatory synapse $2\left(E_{2}\right)$

Inhibitory synapse $\left(1_{1}\right)$

\section{Figure 2.4: Different cases of IPSP and EPSP summation [12].}

Postsynaptic potentials will be the focus of this work. In particular, a method of breaking 
apart a compound PSP (multiple IPSPs and EPSPs) into single IPSPs and EPSPs will be presented and evaluated.

\subsubsection{Action Potentials}

When the membrane potential of a neuron reaches the threshold voltage, an action potential (AP) is fired. APs are the signals that transfer information from the receptive regions of a neuron (the dendrites) to the secretory regions of a neuron (the axon terminals). Whereas a graded potential can be many different shapes and amplitudes - some strong, some weak —an AP is an all-or-nothing event: it either happens completely or not at all. Once an AP is fired, it self-propagates along the axon of the neuron toward the axon's terminals at a constant velocity. This propagation is illustrated in Figure 2.5.

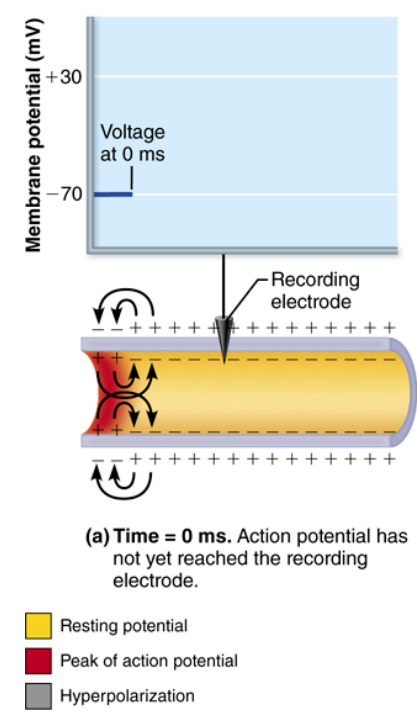

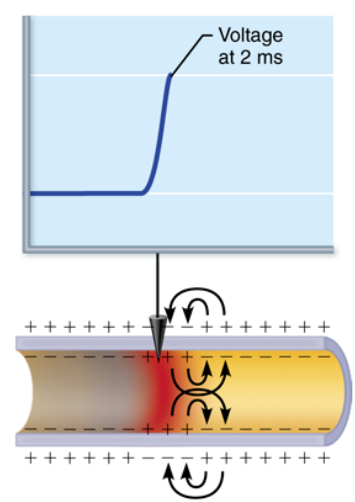

(b) Time $=2 \mathrm{~ms}$. Action potential peak reaches the recording electrode.

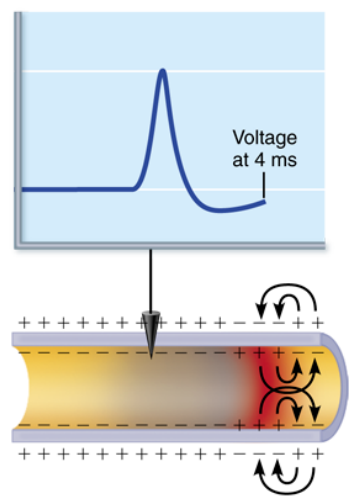

(c) Time $=4 \mathrm{~ms}$. Action potential peak has passed the recording electrode. Membrane at the hyperpolarized.

Figure 2.5: Action potential propagation along an axon [12].

The initiation of an AP in a neuron sets off a domino effect of depolarizations along its axon, such that the peak of the AP travels at a constant speed. A typical shape for an AP can be seen in Figure 2.5(c). 


\subsection{The Synapse}

The nervous system depends on the flow of information through networks of neurons, connected by synapses. A synapse is a gap between two neurons that mediates the transfer of information between one neuron and the next. The neuron conducting an impulse towards the synapse is the presynaptic neuron and the neuron transmitting a signal away from the synapse is the postsynaptic neuron. Most neurons function as both presynaptic and postsynaptic neurons.

Synapses come in two variations: chemical synapses and electrical synapses. At a chemical synapse, the presynaptic neuron releases neurotransmitter molecules, which bind to and open/close corresponding ligand-gated ion channels (Figure 2.6) on the postsynaptic neuron, creating a graded potential.
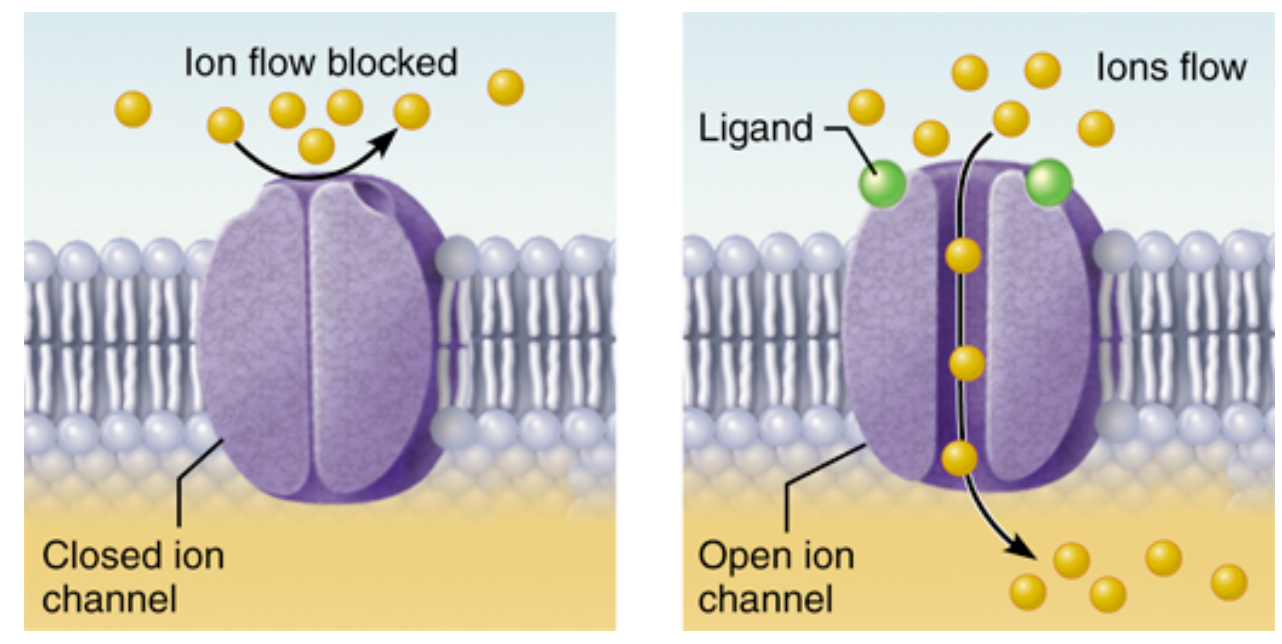

Figure 2.6: Ligand-gated ion channel [12].

Electrical synapses are gap junctions between two neurons, which are faster than chemical synapses, but lack gain. These are much less common than chemical synapses, and will not be considered in this paper.

\subsubsection{Information Transfer Across Chemical Synapses}

Chemical transmission across a synapse always follows a similar chain of events. These steps are listed below, and have corresponding illustrations in Figure 2.7. 
1. An action potential arrives at the axon terminal.

2. Depolarization of the membrane by the AP causes voltage-gated $\mathrm{Ca}^{2+}$ channels to open, allowing $\mathrm{Ca}^{2+}$ to flow into the neuron.

3. $\mathrm{Ca}^{2+}$ causes synaptic vesicles to fuse with the axon membrane and empty their neurotransmitter content into the synaptic cleft.

4. Neurotransmitter diffuses across the synaptic cleft and binds to specific receptors on the postsynaptic neuron's membrane.

5. Neurotransmitter binding causes the ion channels to open or close, resulting in graded potentials (IPSPs and EPSPs).

6. Neurotransmission stops by reuptake through transport proteins, enzymatic degradation, or diffusion away from the synapse. 


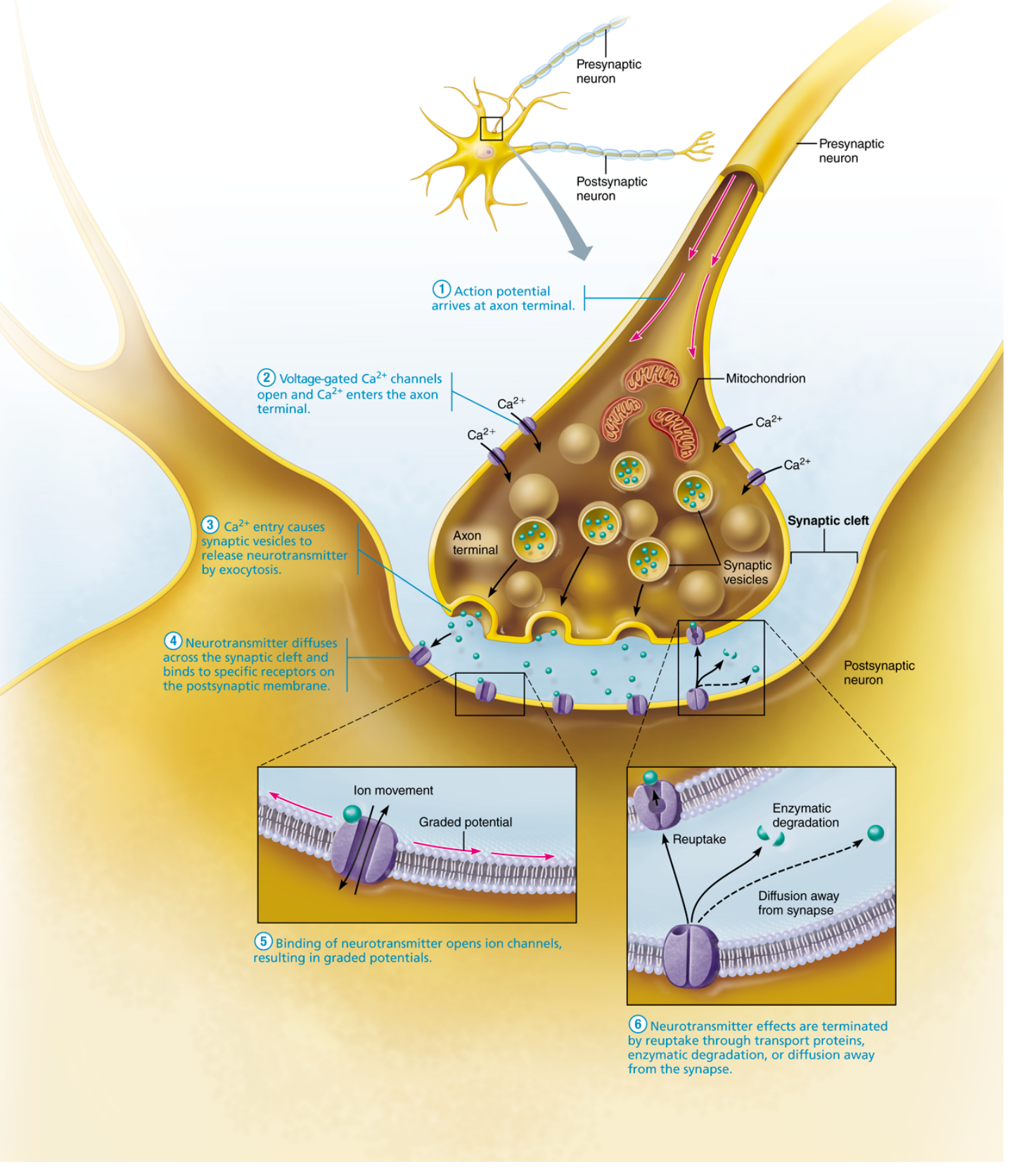

Figure 2.7: Chemical synapse signal transmission [12]. 


\subsubsection{Neurotransmitters}

As seen in the previous section, neurotransmitters are critical for the communication between neurons. However, there is not just one type of neurotransmitter. Rather, they come in many different shapes, sizes, and chemical compositions.

Many neurotransmitters are synthesized from simple precursors such as amino acids. Other neurotransmitters are monoamines, which are amino groups attached to an aromatic ring. Some neurotransmitters are peptides, which are chains of amino acid monomers linked by peptide bonds. Others aren't easily classified, like the organic chemical acetylcholine, which is the primary neurotransmitter used in the muscular system. A survey of the structures of these types of molecules can be seen in Figure 2.8.
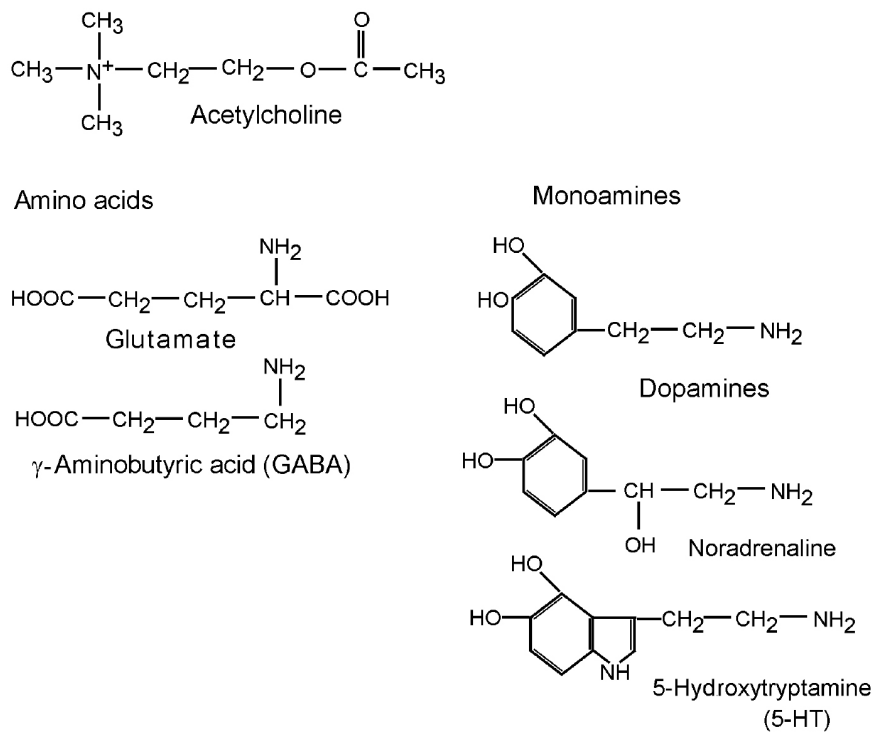

Peptides

Tyr-Gly-Gly-Phe-Met Met-enkephalin

Arg-Pro-Lys-Pro-Gln-Gln-Phe-Phe-Gly-Leu-Met-NH 2

Substance $P$

$\mathrm{Arg}=$ arginine, Gly $=$ glycine, $\mathrm{Gln}=$ glutamine, Leu = leucine, Lys = lysine,

$\mathrm{Phe}=$ phenylalanine, Pro $=$ proline, Met $=$ methionine

Figure 2.8: Structural formulas of common neurotransmitters [13].

For a substance to be considered a neurotransmitter, it must meet the following criteria, from Purves et al.:

1. The substance must be present within the presynaptic neuron 
2. The substance must be released in response to presynaptic depolarization via $\mathrm{Ca}^{2+}$

3. Specific receptors for the substance must be on the postsynaptic neuron [14]

Though all neurotransmitters meet these criteria and follow the same steps when causing a postsynaptic potential, each neurotransmitter has a different function in the body. Of particular interest in this work are glutamate and gamma-Aminobutyric acid (GABA), as they are the primary excitatory and inhibitory neurotransmitters of the mammalian brain, respectively [15][16].

\subsubsection{Glutamate}

Glutamate plays a key role in synaptic plasticity, or the ability for synapses to strengthen or weaken over time, and thus is involved in cognitive functions such as learning and memory [17]. Glutamate has also been proven to be beneficial for several neurological disorders including epilepsy and intellectual disability [18].

\subsubsection{GABA}

GABA acts as a counterbalance to the excitatory effects of glutamate by inhibiting the transfer of nerve impulses between neurons. In both human and animal studies, it has been shown that positive modulators of GABA receptors inhibit anxiety while negative modulators cause anxiety [19]. Several other clinical studies indicate that not only anxiety disorders, but also depressive disorders depend on GABA metabolism [19].

\subsubsection{Neurotransmitter Receptors}

During synaptic transmission, a neurotransmitter will bind to one of two broad families of receptor proteins. The first family of receptors are ionotropic receptors, which are directly connected to ion channels; they are synonymous with ligand-gated ion channels. The second family of receptors are metabotropic receptors, which depend on a cascade of metabolic steps to open an ion channel. These receptors activate G-proteins, which then disassociate from the receptor to interact with an ion channel or bind to other effector 
proteins to eventually open or close an ion channel. These two families of neurotransmitter receptors are compared in Figure 2.9.

(A) Ligand-gated ion channels

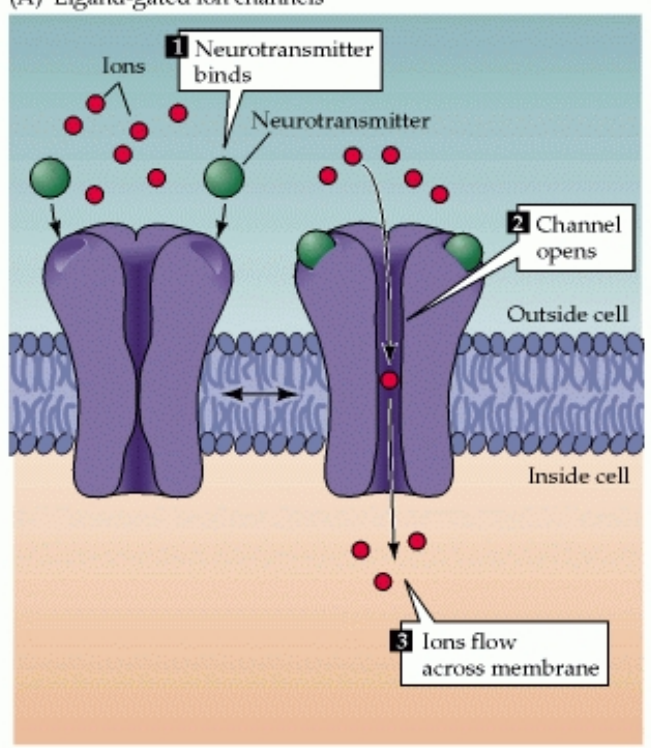

(B) G-protein-coupled receptors

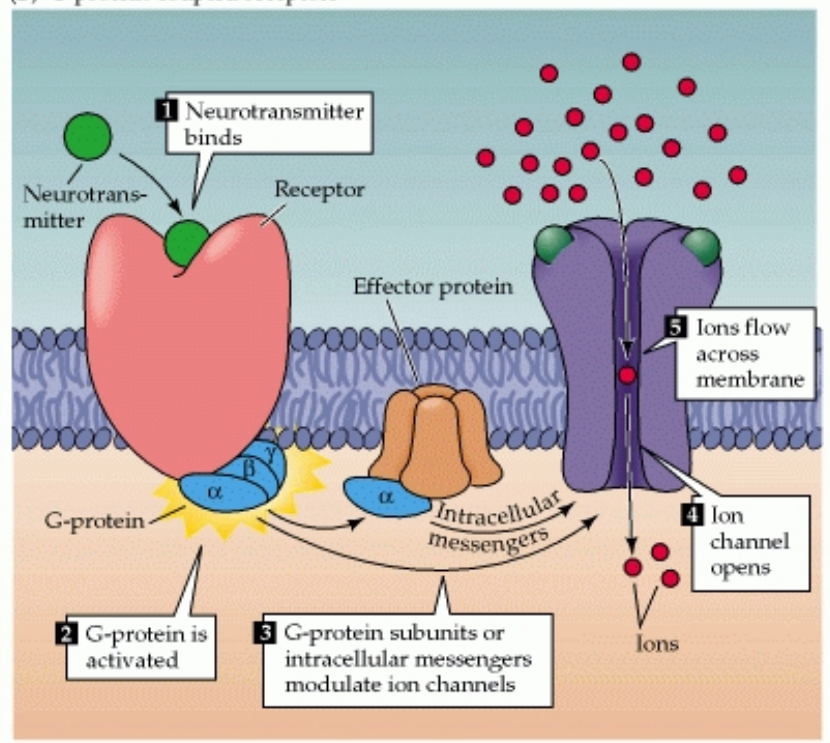

Figure 2.9: Ionotropic and metabotropic neurotransmitter receptors [14].

In this study, only ionotropic glutamate receptors and ionotropic GABA receptors will be considered. The following four receptors will be considered:

1. Glutamate receptors
(a) AMPA receptor
(b) NMDA receptor

2. GABA receptors
(a) Fast-responding $\mathrm{GABA}_{\mathrm{A}}$ receptor $\left(\mathrm{GABA}_{\mathrm{A}}\right.$, fast $)$
(b) Slow-responding $\mathrm{GABA}_{\mathrm{A}}$ receptor $\left(\mathrm{GABA}_{\mathrm{A} \text {, slow }}\right)$

The $\alpha$-amino-3-hydroxy-5-methyl-4-isoxazolepropionic acid (AMPA) receptor is an ionotropic glutamate receptor, named as such because the compound AMPA is a selective agonist for the receptor. AMPA trafficking (relocation of AMPA receptors from one region of a cell to another) is known to play an important role in synaptic plasticity [20]. In many neuropathies, AMPA receptor function is altered, making it a potential therapeutic target. 
The N-methyl-D-aspartate (NMDA) receptor is an ionotropic glutamate receptor, named as such because the agonist molecule NMDA selectively binds to it. The NMDA receptor is essential for the synaptic plasticity underlying learning, memory and development [21]. It is also associated with a number of diseases. A severe form of encephalitis (acute inflammation of the brain) has been found to be caused by antibodies against NR1-NR2 heteromers of the NMDA receptor [22]. This is called anti-NMDA-receptor encephalitis, which was discovered in 2007. Several NMDAergic drugs have been created, such as the NMDA receptor agonist metamine, which is the first in a novel class of Alzheimer's disease medications [23].

The neurotransmitter GABA mediates most neural inhibition through $\mathrm{GABA}_{\mathrm{A}}$ receptors [24]. Changes in GABAergic transmission contribute to the etiology of several neurological and mental disorders, such as epilepsy, anxiety, Angelman syndrome, and schizophrenia [24]. Thus, there is a strong case for the development of GABAergic drugs, especially those targeting $\mathrm{GABA}_{\mathrm{A}}$ receptors. Two kinetically distinct evoked $\mathrm{GABA}_{\mathrm{A}}$ responses $\left(\mathrm{GABA}_{\mathrm{A}}\right.$, fast and $\mathrm{GABA}_{\mathrm{A} \text {, slow }}$ ) have been observed in neurons [25], and as such will be treated as different receptors in this work.

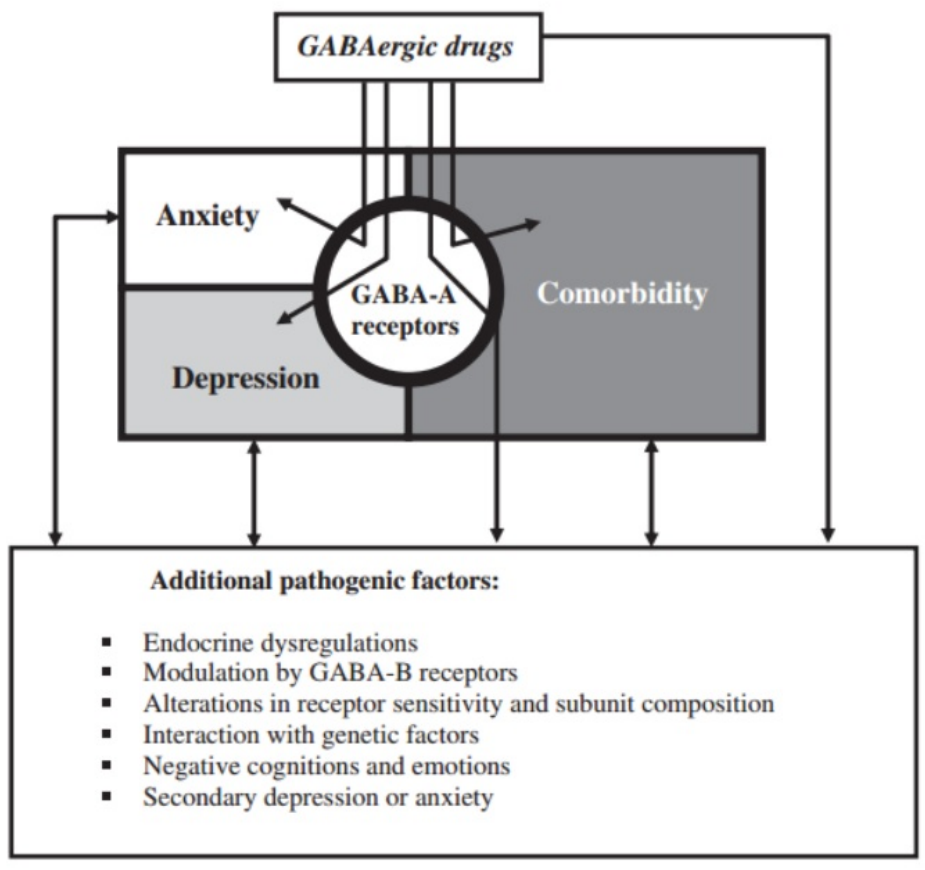

Figure 2.10: GABAergic drugs as a treatment of stress-related disorders [19]. 


\section{Methods}

Given a recording of a postsynaptic potential in a neuron, it is difficult to determine what neurotransmitters caused the potential, since it is often a summation of many different potentials. This paper will describe and evaluate a method of decomposing a compound potential into single-neuron potentials caused by specific types of neurotransmitter receptors. This technique could be used to determine the active synaptic receptors of a given neuron for study or diagnosis of diseases caused by neurotransmission deficiencies.

In general, the presented method is a way of decomposing a compound postsynaptic potential $\Psi(t)$ into $n$ single postsynaptic potentials, $\Lambda_{1}(t), \ldots, \Lambda_{n}(t)$. Functional representations of the single potentials, $\Lambda_{k}(t)$, must be determined before decomposing a compound potential into them. This could be done experimentally by isolating specific receptors in a neuron and stimulating a presynaptic neuron. As a proof of concept, biophysical models found in the literature will be used for these functions.

\subsection{Postsynaptic Potential Models}

Conductance-based models are the simplest biophysically-accurate models for the excitable cell. Graded potentials can be simulated by modeling the neuron's membrane as a capacitor and the influx of ions due to a neurotransmitter-binding event as a transient conductance. The postsynaptic potential in a neuron can be approximated by the following differential equation [26].

$$
C \frac{d v}{d t}=-G_{s y n}(t)\left(v-V_{s y n}\right)-g_{m}\left(v-V_{m}\right)
$$

where $v=v(t)$ is the graded potential, $C$ is the membrane capacitance, $G_{s y n}(t)$ is the transient synaptic conductance due to a neurotransmitter binding event, $V_{\text {syn }}$ is the synaptic 
reversal potential, $g_{m}$ is the intrinsic membrane conductance, and $V_{m}$ is the resting membrane potential of the neuron. Values used in this equation are outlined in Table 3.1. $G_{\text {syn }}(t)$ and $V_{\text {syn }}$ will be listed later, as they depend on the type of neurotransmitter receptor.

Table 3.1: Values used in Postsynaptic Potential Models.

\begin{tabular}{lll}
\hline Term & Value & Description \\
\hline$C$ & $1 \mathrm{nS}$ & Membrane capacitance[26] \\
$g_{m}$ & $0.0258 \mathrm{\mu S}$ & Intrinsic membrane conductance[26] \\
$V_{m}$ & $-75 \mathrm{mV}$ & Resting membrane potential
\end{tabular}

\subsubsection{Synaptic Conductance Model}

In order to use (3.1) for different synaptic receptors, functions for $G_{\text {syn }}(t)$ were found in the literature, based on curve-fitting to experimental data. Below are equations that model synaptic conductance during the binding of neurotransmitters to four different types of synaptic receptors: AMPA receptors, NMDA receptors, $\mathrm{GABA}_{\mathrm{A}}$, fast receptors and $\mathrm{GABA}_{\mathrm{A}}$, slow receptors [27].

- Excitatory conductances

- AMPA conductance

$$
G_{A}(t)= \begin{cases}\overline{g_{A}} \cdot \text { rate } \cdot t & t<0.5 \mathrm{~ms} \\ \overline{g_{A}} \cdot e^{-t / \tau_{A}} & t \geq 0.5 \mathrm{~ms}\end{cases}
$$

- NMDA conductance

$$
G_{N}(t)=\overline{g_{N}} \frac{e^{-t / \tau_{N 1}}-e^{-t / \tau_{N 2}}}{1+\eta \cdot\left[\mathrm{Mg}^{2+}\right] \cdot e^{-\gamma \cdot V_{m}}}
$$

- Inhibitory conductances

- $\mathrm{GABA}_{\mathrm{A} \text {, fast }}$ conductance

$$
G_{F}(t)=\overline{g_{F}} \cdot\left[1-e^{-t / \tau_{F 1}}\right] \cdot e^{-t / \tau_{F 2}}
$$


- GABA $_{\mathrm{A}, \text { slow }}$ conductance

$$
G_{S}(t)=\overline{g_{S}} \cdot\left[1-e^{-t / \tau_{S 1}}\right] \cdot e^{-t / \tau_{S 2}}
$$

These equations were used as substitutes for $G_{\text {syn }}(t)$ in (3.1) to produce four independent equations. Since the ensuing decomposition methods assume linear summation, the molar concentration rate, $\eta$, in (3.3) was set to $0 / \mathrm{mM}$ to make the equation voltage-independent. Table 3.2 outlines the other values used for these conductance equations.

Table 3.2: Values used in Synaptic Conductance Models.

\begin{tabular}{|c|c|c|}
\hline Term & Value & Description \\
\hline$\overline{g_{A}}$ & $0.3 \mathrm{nS}$ & AMPA max synaptic conductance \\
\hline$\tau_{A}$ & $2 \mathrm{~ms}$ & AMPA time constant $[27]$ \\
\hline rate & $2 \mathrm{~ms}^{-1}$ & AMPA rate[27] \\
\hline$V_{\text {syn }, A}$ & $0 \mathrm{mV}$ & AMPA synaptic reversal potential[27] \\
\hline$\overline{g_{N}}$ & $0.3 \mathrm{nS}$ & NMDA max synaptic conductance \\
\hline$\tau_{N 1}$ & $60 \mathrm{~ms}$ & NMDA time constant $1[27]$ \\
\hline$\tau_{N 2}$ & $0.66 \mathrm{~ms}$ & NMDA time constant $2[27]$ \\
\hline$\eta$ & $0 / \mathrm{mM}$ & Molar rate constant $[27]$ \\
\hline $\mathrm{Mg}^{2+}$ & $1 \mathrm{mM}$ & $\mathrm{Mg}^{2+}$ concentration $[27]$ \\
\hline$\gamma$ & $0.08 \mathrm{mV}^{-1}$ & Exponential decay rate $[27]$ \\
\hline$V_{\text {syn }, N}$ & $0 \mathrm{mV}$ & NMDA synaptic reversal potential[27] \\
\hline$\overline{g_{S}}$ & $2 \mathrm{nS}$ & $\mathrm{GABA}_{\mathrm{A}, \text { slow }}$ max synaptic conductance \\
\hline$\tau_{S 1}$ & $0.75 \mathrm{~ms}$ & $\mathrm{GABA}_{\mathrm{A}, \text { slow }}$ time constant $1[27]$ \\
\hline$\tau_{S 2}$ & $37 \mathrm{~ms}$ & $\mathrm{GABA}_{\mathrm{A}, \text { slow }}$ time constant $2[27]$ \\
\hline$V_{\text {syn }, G}$ & $-80 \mathrm{mV}$ & $\mathrm{GABA}_{\mathrm{A}}$ synaptic reversal potential $[27]$ \\
\hline$\overline{g_{F}}$ & $2 \mathrm{nS}$ & $\mathrm{GABA}_{\mathrm{A} \text {, fast }}$ max synaptic conductance \\
\hline$\tau_{F 1}$ & $1.5 \mathrm{~ms}$ & $\mathrm{GABA}_{\mathrm{A}, \text { fast }}$ time constant $1[27]$ \\
\hline$\tau_{F 2}$ & $7.25 \mathrm{~ms}$ & $\mathrm{GABA}_{\mathrm{A}, \text { fast }}$ time constant $2[27]$ \\
\hline
\end{tabular}




\subsubsection{Solving for Postsynaptic Potential Numerically}

To determine the postsynaptic potentials for these four different types of synaptic receptors, the differential equation (3.1) needed to be solved. This first-order ordinary differential equation (ODE) was solved numerically using MATLAB's ode15s solver. The solver required the equations to be written in this form:

$$
\frac{d \mathbf{y}(t)}{d t}=\mathbf{f}(\mathbf{y}(t), t)
$$

where $\mathbf{y}(t)$ is a vector of functions and $\mathbf{f}$ is a vector-valued function. The equations modeling postsynaptic potentials for each of the four synaptic receptors were programmed into the solver as follows:

$$
\begin{aligned}
& \frac{d v_{A}}{d t}=\frac{1}{C}\left(-G_{A}(t)\left(v_{A}-V_{s y n, A}\right)-g_{m}\left(v_{A}-V_{m}\right)\right) \\
& \frac{d v_{N}}{d t}=\frac{1}{C}\left(-G_{N}(t)\left(v_{N}-V_{s y n, N}\right)-g_{m}\left(v_{N}-V_{m}\right)\right) \\
& \frac{d v_{F}}{d t}=\frac{1}{C}\left(-G_{F}(t)\left(v_{F}-V_{s y n, G}\right)-g_{m}\left(v_{F}-V_{m}\right)\right) \\
& \frac{d v_{S}}{d t}=\frac{1}{C}\left(-G_{S}(t)\left(v_{S}-V_{s y n, G}\right)-g_{m}\left(v_{S}-V_{m}\right)\right)
\end{aligned}
$$

These equations were solved for $v_{A}(t), v_{N}(t), v_{F}(t)$, and $v_{S}(t)$, respectively. Figure 3.1 shows a plot of each of the voltages, normalized by subtracting the base voltage $V_{m}$ from each solution. 


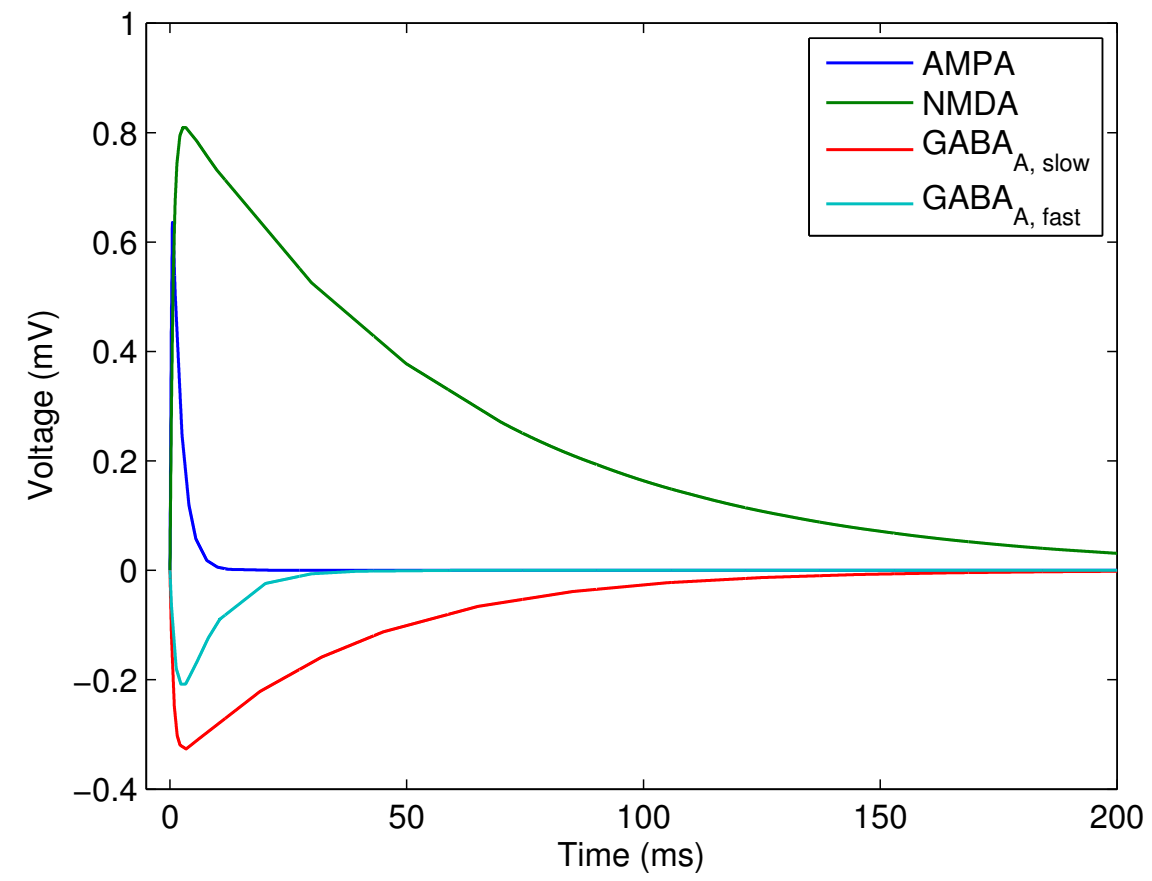

Figure 3.1: Postsynaptic Potentials due to binding to four different types of receptors: AMPA, NMDA, GABA $A_{A}$, fast and $\mathrm{GABA}_{\mathrm{A} \text {, slow. }}$.

\subsection{Generalized Fourier series approach}

A natural way to decompose a compound signal into smaller signals, or basis functions, is through the generalized Fourier series. However, since in general the single postsynaptic potentials $\Lambda_{k}(t)$ do not form an orthonormal basis, this will only provide a rough estimation. Equation 3.6 can be used to estimate the amount $\xi_{k}^{\prime}$ of the single potential $\Lambda_{k}(t)$ in the compound potential, where $\left\|\Lambda_{k}(t)\right\|^{2}=\int_{0}^{\tau_{f}} \Lambda_{k}(t) \Lambda_{k}(t) d t$.

$$
\xi_{k}^{\prime}=\frac{1}{\left\|\Lambda_{k}(t)\right\|^{2}} \int_{0}^{\tau_{f}} \Psi(t) \Lambda_{k}(t) d t
$$

This typically yields incorrect estimates since the single potentials that make up a compound potential are usually temporally overlapped, and thus not orthogonal or even nearorthogonal. 


\subsection{Perturbation Decomposition Method}

Szlavik [28] presented a novel technique for estimating the size distribution of nerve fibers contributing to a compound-evoked potential, $\Psi(t)$. The technique uses functional representations for single fiber-evoked potentials from $n$ different fiber diameter classes, $\Lambda_{1}(t), \ldots, \Lambda_{n}(t)$, found in the literature. The compound-evoked potential in the frequency domain $\Psi(f)=$ $\mathcal{F}[\Psi(t)]$ is hypothesized to consist of a contribution from each fiber diameter class $\Lambda_{k}(f)=$ $\mathcal{F}\left[\Lambda_{k}(t)\right]$, which have been perturbed, by a determinable degree $\epsilon_{i, j}$, by contributions from each of the other classes. This statement can be written mathematically as

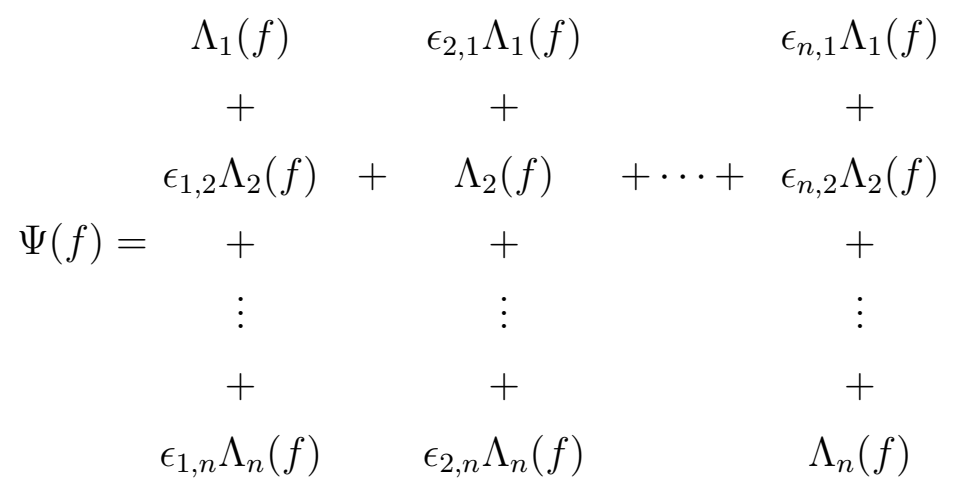

The $k$-th column in (3.7) represents the perturbation of the respective diameter class $\Lambda_{k}(f)$. This equation may be evaluated at a specific frequency point $f_{l}$ for $l=1, \ldots, m$ and rearranged as follows

$$
\Psi\left(f_{l}\right)-\sum_{i=1}^{n} \Lambda_{i}\left(f_{l}\right)=\sum_{\substack{i=1 \\ i \neq j}}^{n} \sum_{j=1}^{n} \epsilon_{i, j} \Lambda_{j}\left(f_{l}\right)
$$

The equations from (3.8) form an overdetermined linear system $\left(m>n^{2}-n\right)$. Thus, an approximate solution can be found in the least-squares sense. It is useful to write (3.7) as a summation over the rows as follows

$$
\begin{aligned}
\Psi(f) & =\sum_{k=1}^{n} B_{k}^{\prime} \Lambda_{k}(f) \\
B_{k}^{\prime} & =\left(\epsilon_{1, k}+\cdots+\epsilon_{k-1, k}+1+\epsilon_{k+1, k}+\cdots+\epsilon_{n, k}\right)
\end{aligned}
$$


where the estimator $B_{k}^{\prime}$ is complex, since the $\epsilon_{i, j}$ are complex valued. The real valued estimator $\beta_{k}^{\prime}$ can be written as

$$
\beta_{k}^{\prime}=\sqrt{\left(B_{k}^{\prime}\right) \cdot\left(B_{k}^{\prime}\right)^{*}}
$$

Once the $\epsilon_{i, j}$ are found, in the least-squares sense, the compound evoked-potential in the frequency domain, $\Psi(f)$, can be approximated as a sum of single fiber-evoked potentials from each fiber diameter class, each scaled linearly by the real valued estimator $\beta_{k}^{\prime}$. An inverse Fourier transform yields an estimation of $\Psi(t)$.

$$
\begin{aligned}
& \Psi(f) \approx \beta_{1}^{\prime} \Lambda_{1}(f)+\beta_{2}^{\prime} \Lambda_{2}(f)+\cdots+\beta_{n}^{\prime} \Lambda_{n}(f) \\
& \Psi(t)=\mathcal{F}^{-1}[\Psi(f)]
\end{aligned}
$$

In effect, this technique approximates the amount of each single fiber-evoked potential in the compound signal $\Psi(t)$. Thus, it can be used to "decompose" a compound fiber evokedpotential into many single fiber-evoked potentials.

\subsubsection{Generalizing the Perturbation Decomposition Method}

Though this method was originally used for estimation of conducting nerve fiber diameter distributions, it can be applied to any situation where a function $\Psi(t)$ needs to be decomposed into $n$ smaller functions $\Lambda_{1}(t), \ldots, \Lambda_{n}(t)$, which may or may not be orthogonal.

A MATLAB function provided by Szlavik can be used to decompose a discrete function represented by the vector $\boldsymbol{\Psi}$ into basis functions $\boldsymbol{\Lambda}_{\mathbf{1}}, \boldsymbol{\Lambda}_{\mathbf{2}}, \ldots, \boldsymbol{\Lambda}_{\mathrm{n}}$. It returns a vector of frequencies $\mathbf{f}=\left[\beta_{1}^{\prime}, \beta_{2}^{\prime}, \ldots, \beta_{n}^{\prime}\right]$, which is a least-squares estimate of the contribution of each basis function to the compound function. Thus, given well-posed inputs, it is expected that $\mathbf{f} \cdot\left[\boldsymbol{\Lambda}_{\mathbf{1}}, \boldsymbol{\Lambda}_{\mathbf{2}}, \ldots, \boldsymbol{\Lambda}_{\mathbf{n}}\right] \approx \mathbf{\Psi}$. This procedure is written in pseudocode as Algorithm 1 . 


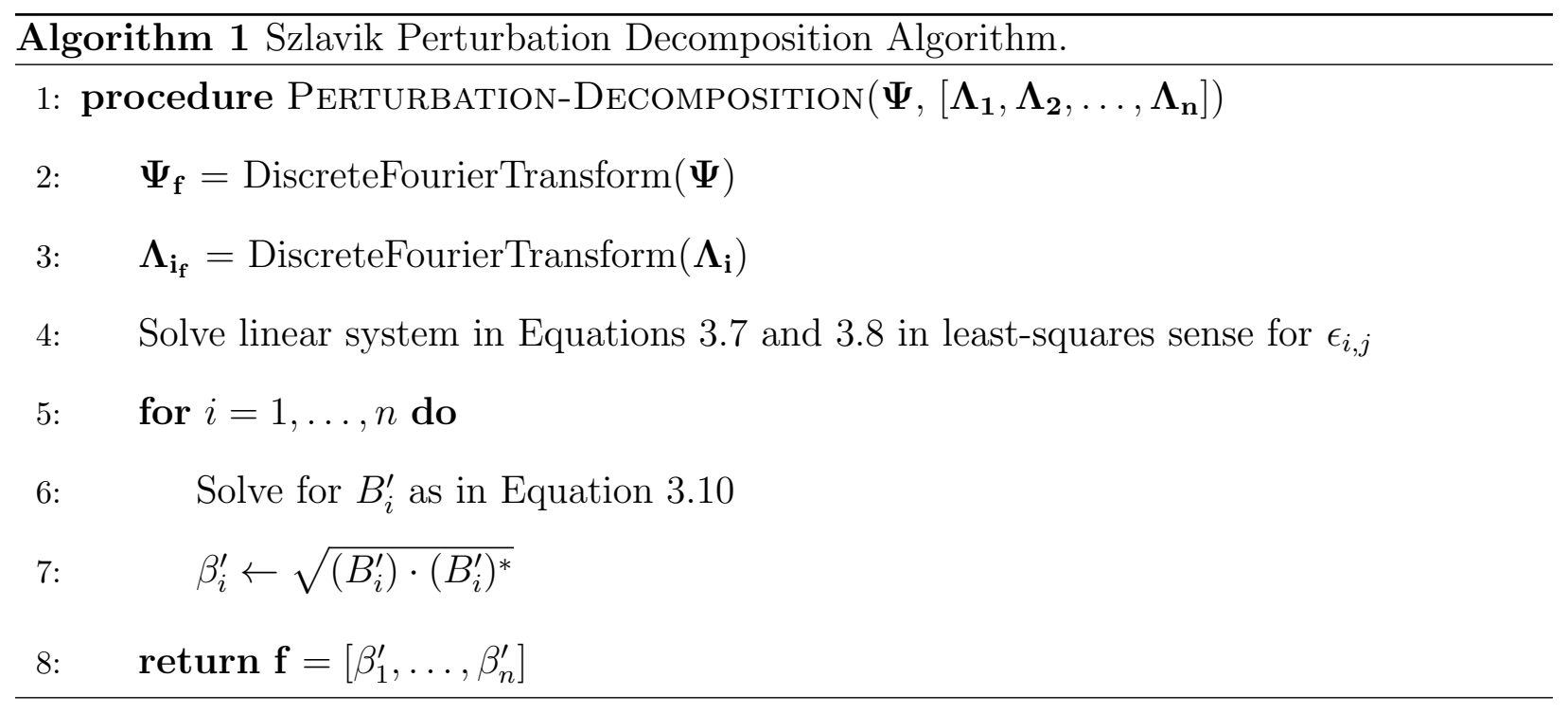

\subsection{Decomposition of Compound Postsynaptic Potential}

The decomposition technique by Szlavik can be applied to compound postsynaptic potentials (PSPs). Given a compound PSP and a set of individual graded PSPs caused by presynaptic neurons, the technique can estimate the amount of each individual PSP in the compound PSP. In this paper, two situations are considered: when all presynaptic neurons fire at once, and when the presynaptic neurons fire at different times.

\subsubsection{Synchronous presynaptic firing}

Assuming that a compound postsynaptic potential was caused by presynaptic neurons that fired at the same time, the compound potential can be written as a linear combination of the individual potentials, $v_{A}(t), v_{N}(t), v_{F}(t)$ and $v_{s}(t)$. The decomposition technique will solve for the coefficients in the following expression:

$$
\Psi(f) \approx \beta_{1}^{\prime} v_{A}(f)+\beta_{2}^{\prime} v_{N}(f)+\beta_{3}^{\prime} v_{F}(f)+\beta_{4}^{\prime} v_{S}(f)
$$

where the functions have been normalized by subtracting the resting membrane voltage in the time-domain. A reconstruction of the compound potential would therefore be $\Psi(t)+V_{m}$. This is an accurate model of graded potential summation if the following assumptions hold:

- Linear summation of postsynaptic potentials 
- Summation occurs at subthreshold neuron voltage

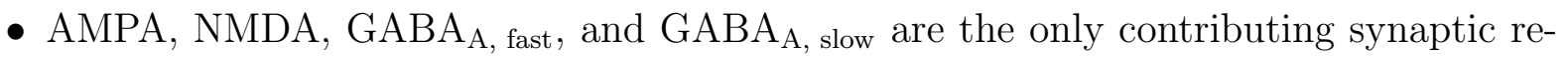
ceptors

- All presynaptic neurons fire at the same time

- Small neuron body (spatial effects are negligible)

\subsubsection{Asynchronous presynaptic firing}

The assumption that all presynaptic neurons fire at the same time is very limiting since different neurons typically fire at different times. However, it was too computationally taxing to consider the most general case where all four receptors are activated at different times. Instead, each of the four types of receptors were considered separately.

\subsubsection{AMPA-mediated potentials}

A compound potential caused by asynchronous AMPA-mediated neurons can be written as

$$
\Psi(t) \approx \beta_{1}^{\prime} v_{A}\left(t-t_{1}\right)+\beta_{2}^{\prime} v_{A}\left(t-t_{2}\right)+\cdots+\beta_{p}^{\prime} v_{A}\left(t-t_{p}\right)
$$

where presynaptic neurons are fired at times $t_{1}, t_{2}, \ldots, t_{p}$. The method from section 3.1.2 will solve for the coefficients in the following expression:

$$
\Psi(f) \approx \sum_{i=1}^{p} \beta_{i}^{\prime} v_{A, t_{i}}(f)
$$

where $v_{A, t_{i}}(f)=\mathcal{F}\left[v_{A}\left(t-t_{i}\right)\right]$ and each function $v_{A}\left(t-t_{i}\right)$ has been normalized by subtracting the resting membrane voltage $V_{m}$. This is an accurate model of graded potential summation if the following assumptions hold:

- Only temporal summation

- Linear summation of postsynaptic potentials

- Summation occurs at subthreshold neuron voltage 
- AMPA is the only contributing synaptic receptor

- Small neuron body (spatial effects are negligible)

Decomposition of potentials caused by the other three receptors follow the exact same steps. The equations for the compound potentials $\Psi(t)$ are listed below.

\subsubsection{GABA $_{\mathrm{A} \text {, slow-mediated potentials }}$}

$$
\Psi(t) \approx \beta_{1}^{\prime} v_{S}\left(t-t_{1}\right)+\beta_{2}^{\prime} v_{S}\left(t-t_{2}\right)+\cdots+\beta_{p}^{\prime} v_{S}\left(t-t_{p}\right)
$$

\subsubsection{GABA $_{\mathrm{A}, \text { fast-mediated potentials }}$}

$$
\Psi(t) \approx \beta_{1}^{\prime} v_{F}\left(t-t_{1}\right)+\beta_{2}^{\prime} v_{F}\left(t-t_{2}\right)+\cdots+\beta_{p}^{\prime} v_{F}\left(t-t_{p}\right)
$$

\subsubsection{NMDA-mediated potentials}

$$
\Psi(t) \approx \beta_{1}^{\prime} v_{N}\left(t-t_{1}\right)+\beta_{2}^{\prime} v_{N}\left(t-t_{2}\right)+\cdots+\beta_{p}^{\prime} v_{N}\left(t-t_{p}\right)
$$




\section{Results}

As seen previously, there were two types of compound potentials that were evaluated: those caused by different neurotransmitter receptors activated at the same time (synchronous presynaptic firing), and those caused by a single type of neurotransmitter receptor activated at different times (asynchronous presynaptic firing). These results are in Sections 4.1 and 4.2 , respectively. Each simulated compound potential was decomposed with Szlavik's perturbation decomposition method, and then with the generalized Fourier series method. The errors from these two methods were then compared. Finally, the perturbation decomposition method is validated with experimental postsynaptic potential data in Section 4.3.

\subsection{Synchronous presynaptic firing}

To demonstrate the effectiveness of the proposed technique, an arbitrary compound potential $\Phi_{P S P}(t)$ was created using four basis potentials.

$$
\Phi_{P S P}(t)=3 v_{A}(t)+12 v_{N}(t)+15 v_{S}(t)+9 v_{F}(t)
$$

This compound potential can be thought of as 3 AMPA-mediated potentials, 12 NMDA-

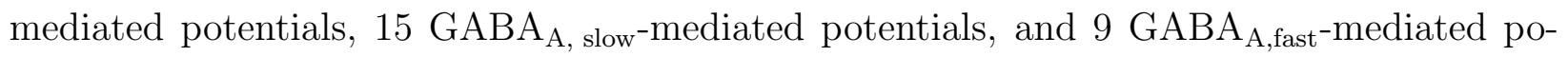
tentials, all occurring at the same time. This combination was selected to demonstrate the effectiveness of the technique rather than to be consistent with any expected physiological pattern. Figure 4.1 shows the basis functions and 4.2 shows the compound potential $\Phi_{P S P}(t)$. 


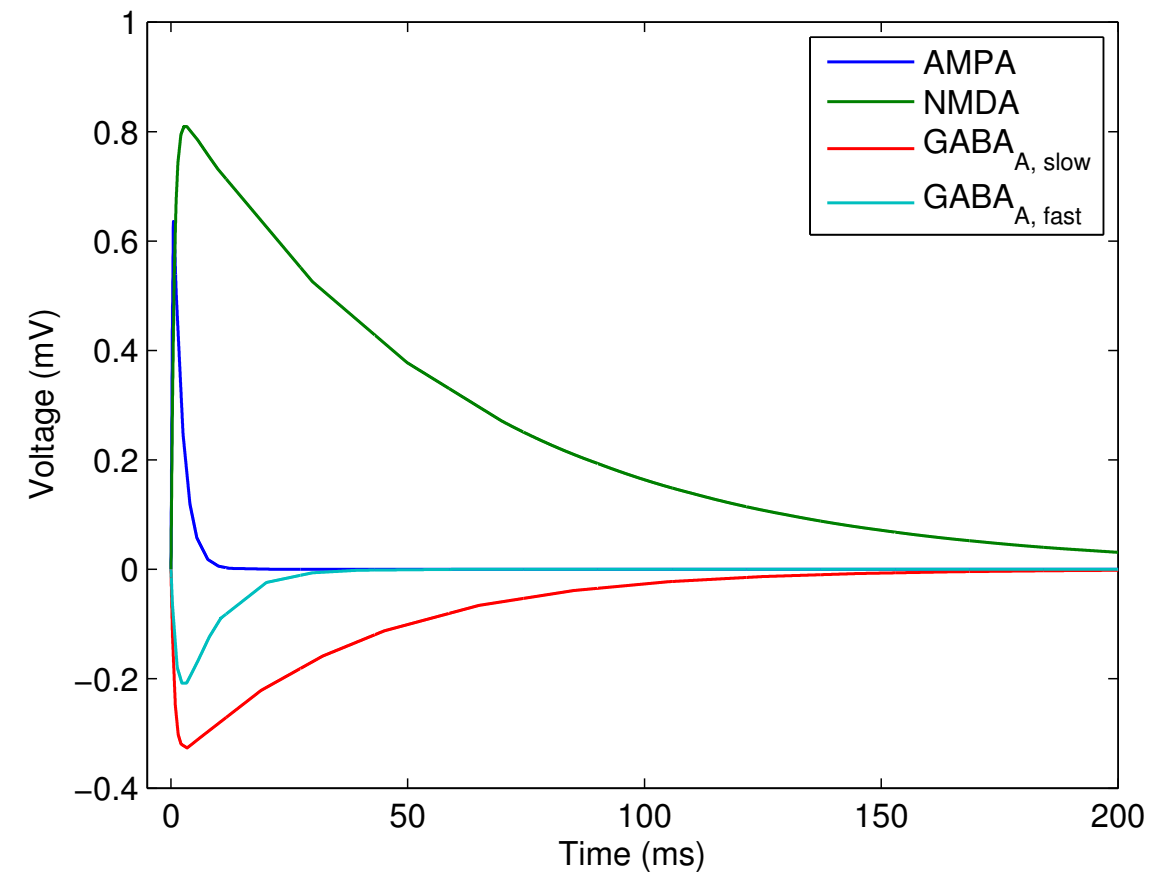

Figure 4.1: Basis functions for synchronous presynaptic firing, $v_{A}(t), v_{N}(t), v_{S}(t), v_{F}(t)$

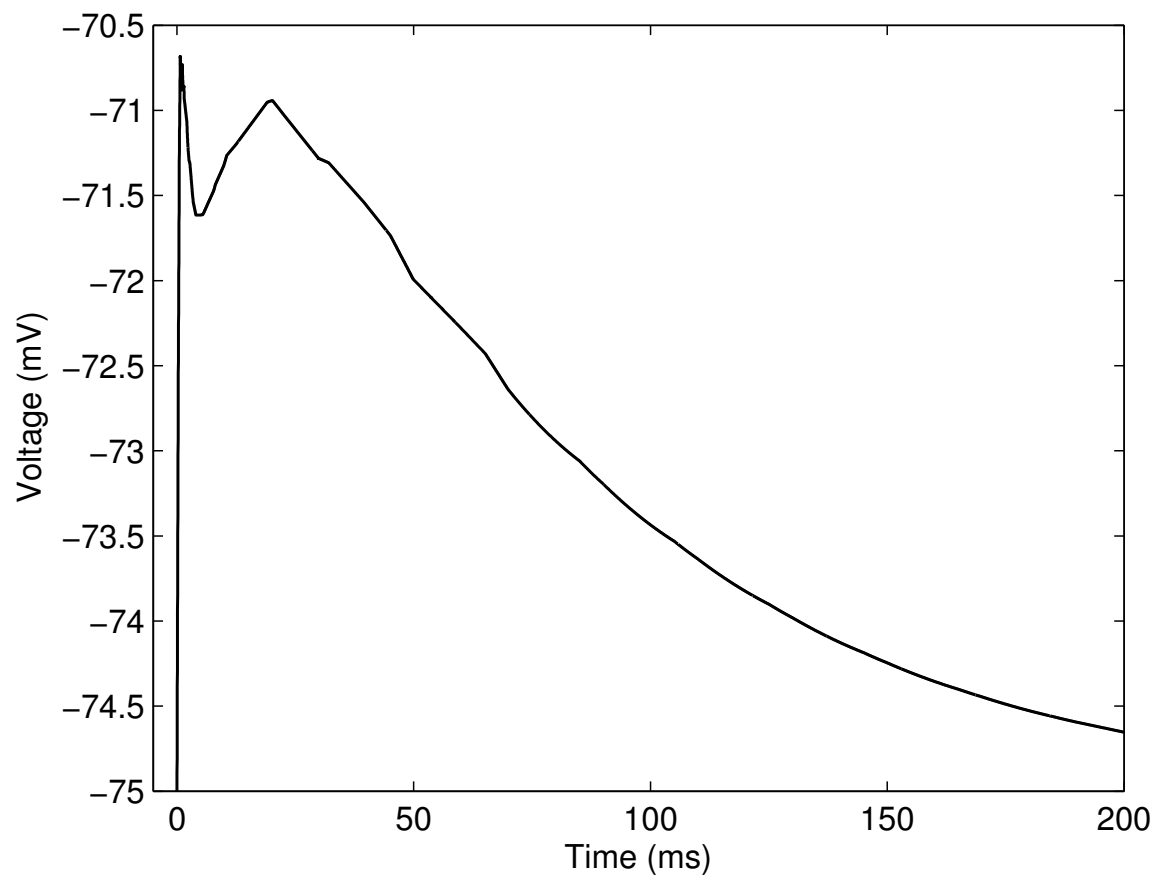

Figure 4.2: Compound function $\Phi_{P S P}(t)+V_{m}$ 
Szlavik's algorithm was run on the compound potential $\boldsymbol{\Psi}=\Phi_{P S P}(t)$ with basis functions $\boldsymbol{\Lambda}_{\mathbf{1}}=v_{A}(t), \boldsymbol{\Lambda}_{\mathbf{2}}=v_{N}(t), \boldsymbol{\Lambda}_{\mathbf{3}}=v_{S}(t)$, and $\boldsymbol{\Lambda}_{\mathbf{4}}=v_{F}(t)$. The algorithm returned frequencies $\beta_{1}^{\prime}=3, \beta_{2}^{\prime}=12, \beta_{3}^{\prime}=15$, and $\beta_{4}^{\prime}=9$, which are the exact frequencies of the functions in the original equation. A plot of the exact frequencies $\beta_{k}$ and the estimates $\beta_{k}^{\prime}$ is seen in Figure 4.3.

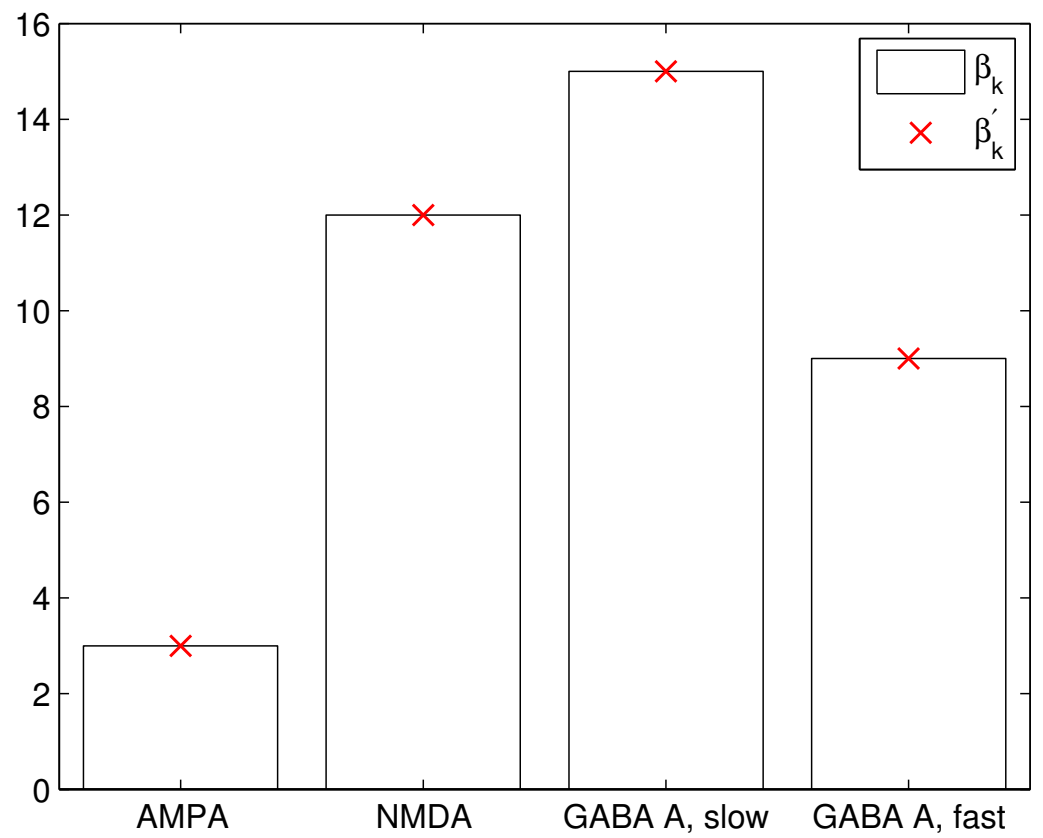

\section{Figure 4.3: Frequency distribution from decomposition of $\Phi_{P S P}(t)$.}

As a comparison, the compound potential was also decomposed using the generalized Fourier series approach, yielding estimates $\xi_{k}^{\prime}$. Because of the non-orthogonality of the basis functions, these estimates were incorrect. A plot comparing error of the Fourier series approach to the Szlavik perturbation decomposition approach can be seen in Figure 4.4. 


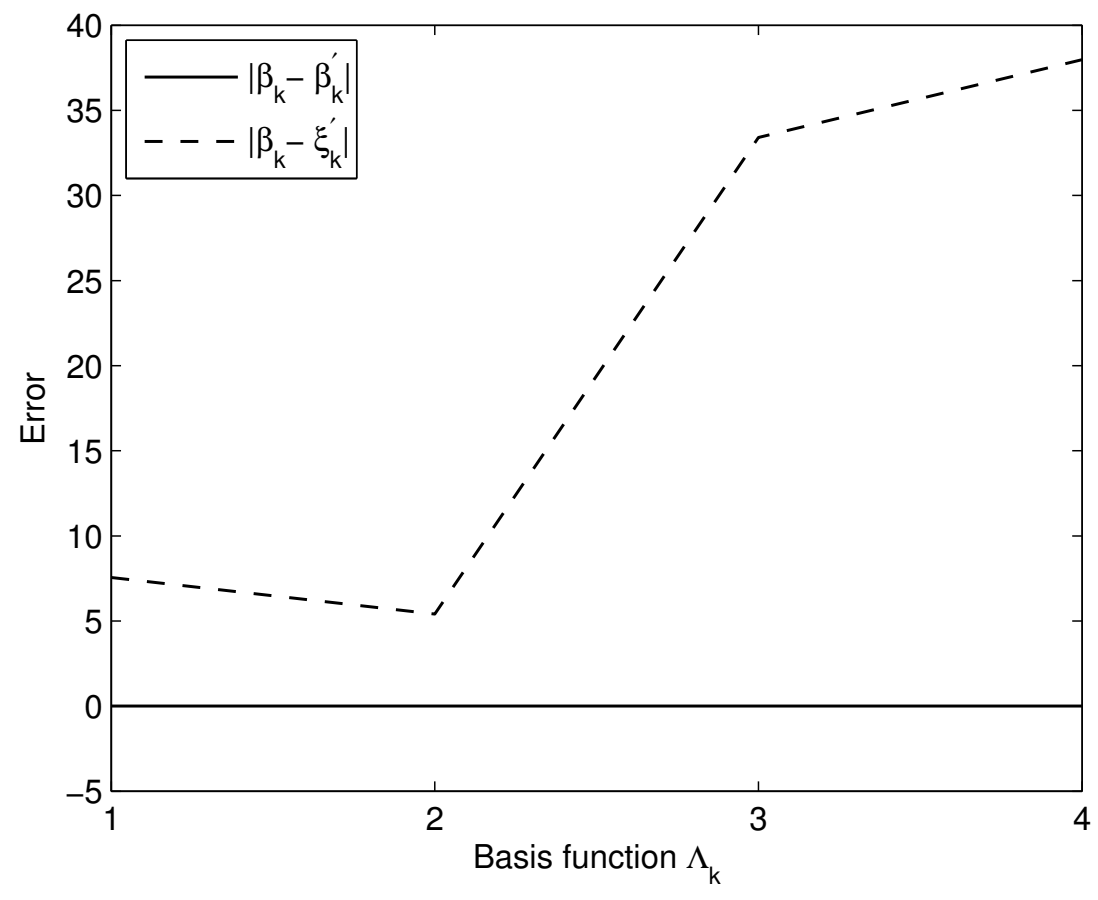

Figure 4.4: Comparison of error from the generalized Fourier series approach $\left(\left|\beta_{k}-\xi_{k}^{\prime}\right|\right)$ to Szlavik perturbation decomposition approach $\left(\left|\beta_{k}-\beta_{k}^{\prime}\right|\right)$.

\subsection{Asynchronous presynaptic firing}

Szlavik's technique was also evaluated for the asynchronous presynaptic firing case. First, AMPA-mediated potentials were considered, then $\mathrm{GABA}_{\mathrm{A} \text {, slow }}$-mediated, then $\mathrm{GABA}_{\mathrm{A}}$, fast ${ }^{-}$ mediated, then finally NMDA-mediated.

\subsubsection{AMPA-mediated potentials}

An arbitrary compound potential $\Phi_{A M P A}(t)$ was created by adding together five time-shifted AMPA-mediated depolarizations, as follows.

$$
\begin{aligned}
\Phi_{A M P A}(t) & =v_{A}\left(t-t_{1}\right)+v_{A}\left(t-t_{2}\right)+v_{A}\left(t-t_{10}\right)+v_{A}\left(t-t_{11}\right)+v_{A}\left(t-t_{30}\right) \\
t_{n} & =(n-1) \cdot 5 \mathrm{~ms}
\end{aligned}
$$

This potential could be caused by a single neuron firing multiple times, or by several 
neurons. The basis functions can be seen in Figure 4.5, and the compound function $\Phi_{A M P A}(t)$ can be seen in Figure 4.6.

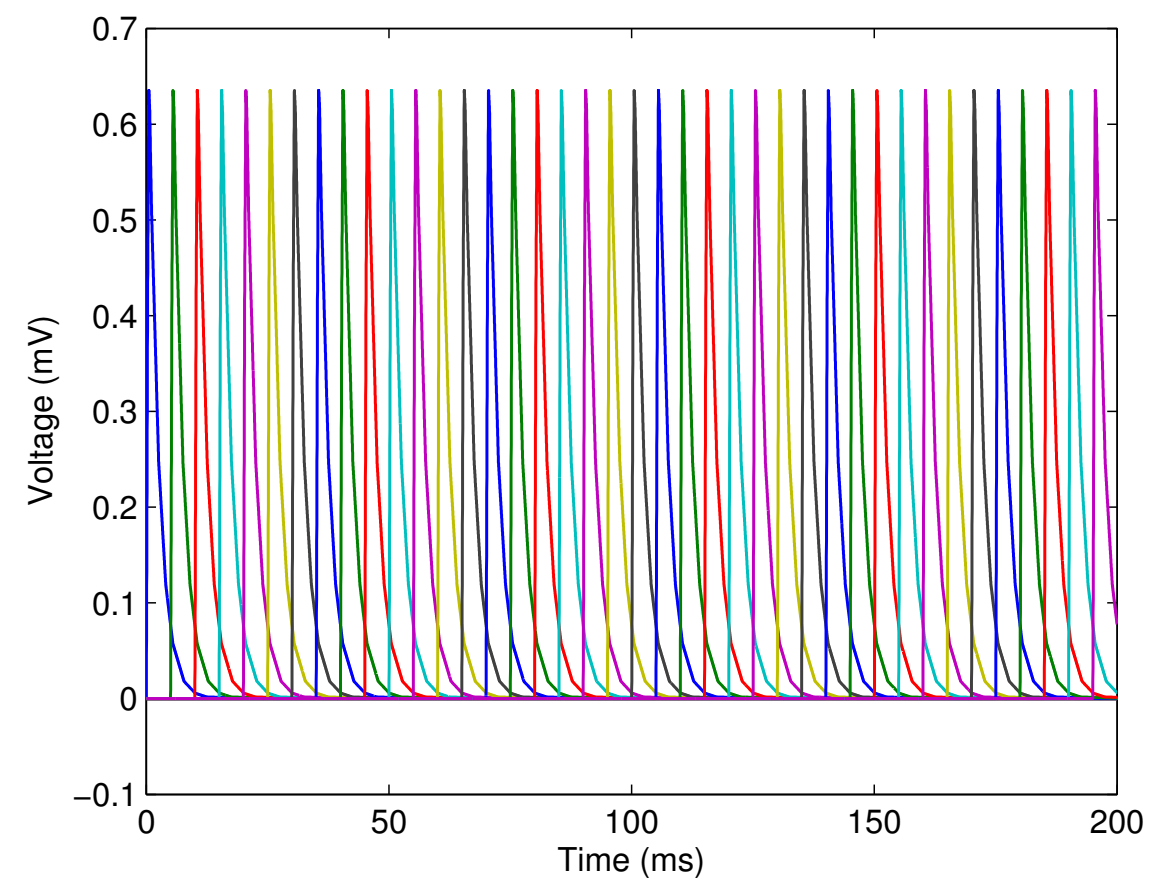

Figure 4.5: Basis functions for asynchronous presynaptic firing of AMPAmediated neurons, $\boldsymbol{\Lambda}_{\mathbf{n}}=v_{A}\left(t-t_{n}\right)$ for $n=1, \ldots, 40$. 


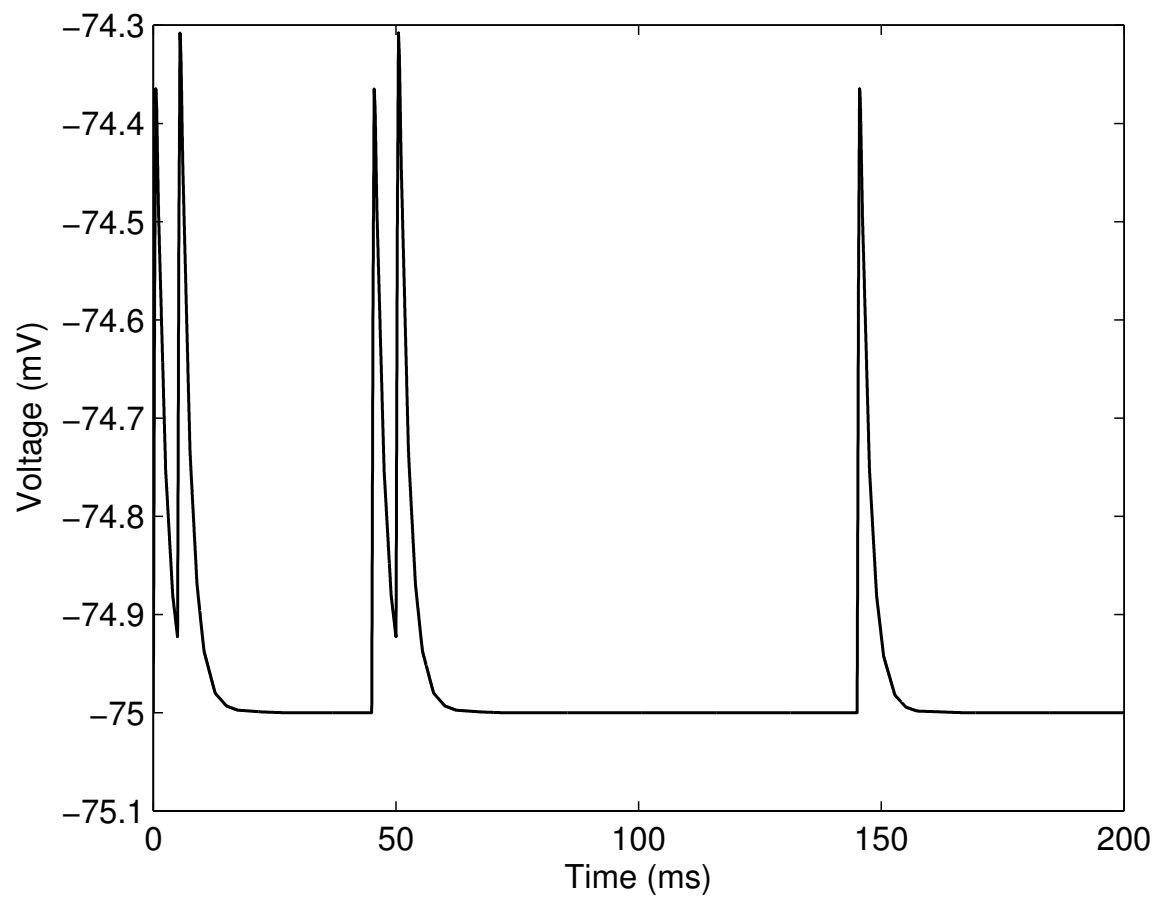

Figure 4.6: Compound function $\Phi_{A M P A}(t)+V_{m}$.

Using Szlavik's algorithm to decompose the signal with $\boldsymbol{\Psi}=\Phi_{A M P A}(t)$ and $\boldsymbol{\Lambda}_{\mathbf{n}}=v_{A}(t-$ $\left.t_{n}\right)$ for $n=1, \ldots, 40$ once again yielded a perfect frequency distribution, with $\beta_{1}^{\prime}=1, \beta_{2}^{\prime}=$ $1, \beta_{10}^{\prime}=1, \beta_{11}^{\prime}=1$, and $\beta_{30}^{\prime}=1$ and all other coefficients equal to zero. A histogram of the frequencies can be seen in Figure 4.7 . 


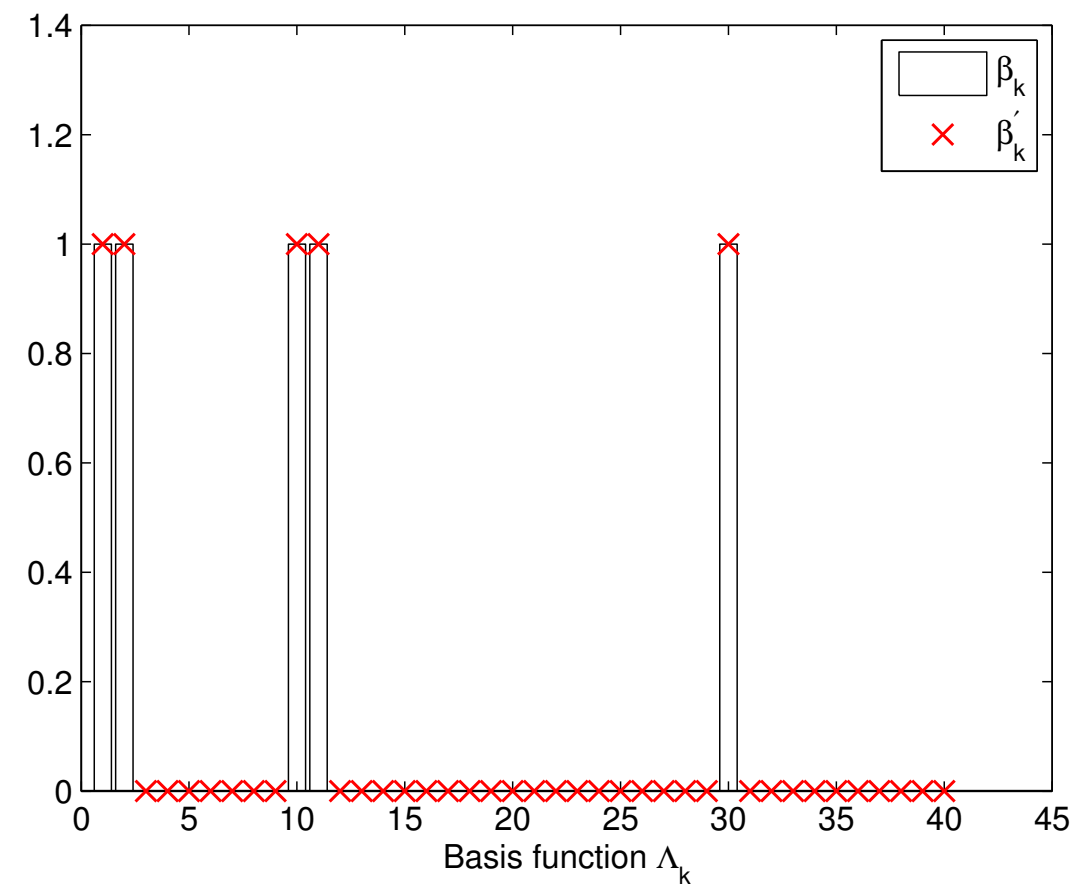

Figure 4.7: Frequency distribution from decomposition of $\Phi_{A M P A}(t)$.

Once again the compound potential $\Phi_{A M P A}(t)$ was decomposed using the generalized Fourier series for comparison. As seen in Figure 4.8, the generalized Fourier series was only marginally worse than the Szlavik perturbation method, which is expected since the basis functions are very distinct. Errors ranged from 0 to about 0.1. 


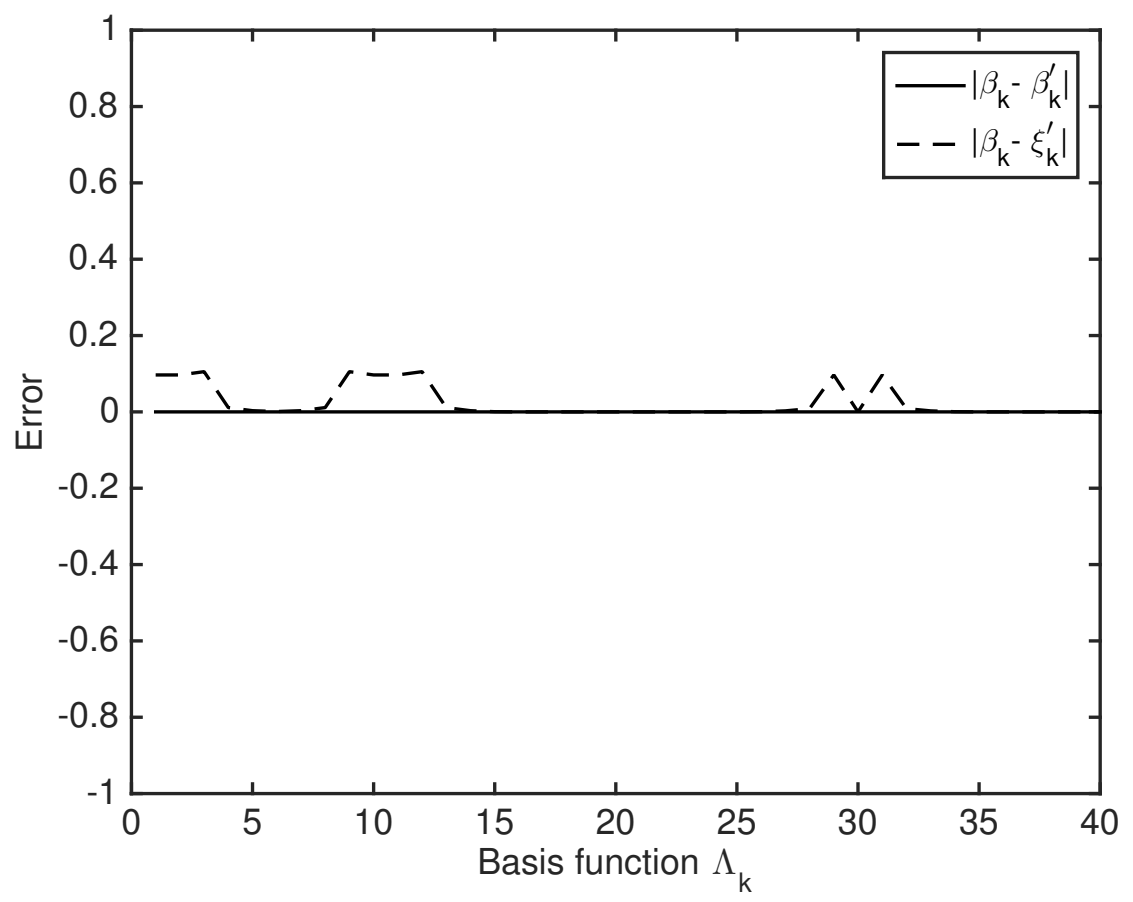

Figure 4.8: Comparison of error from generalized Fourier series approach to Szlavik perturbation decomposition approach for AMPA-mediated potentials.

\subsection{2 $\quad$ GABA $_{\mathrm{A}, \text { slow-mediated potentials }}$}

An arbitrary compound potential $\Phi_{G A B A, \text { slow }}(t)$ was created by adding together four time-

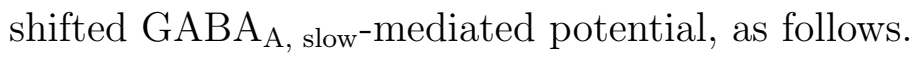

$$
\begin{aligned}
\Phi_{G A B A, \text { slow }}(t) & =v_{S}\left(t-t_{1}\right)+3 v_{S}\left(t-t_{2}\right)+v_{S}\left(t-t_{3}\right)+5 v_{S}\left(t-t_{10}\right) \\
t_{n} & =(n-1) \cdot 10 \mathrm{~ms}
\end{aligned}
$$

The basis functions $v_{S}\left(t-t_{1}\right), \ldots, v_{S}\left(t-t_{20}\right)$ and the compound function $\Phi_{G A B A, \text { slow }}(t)$ can be seen in Figures 4.9 and 4.10, respectively. 


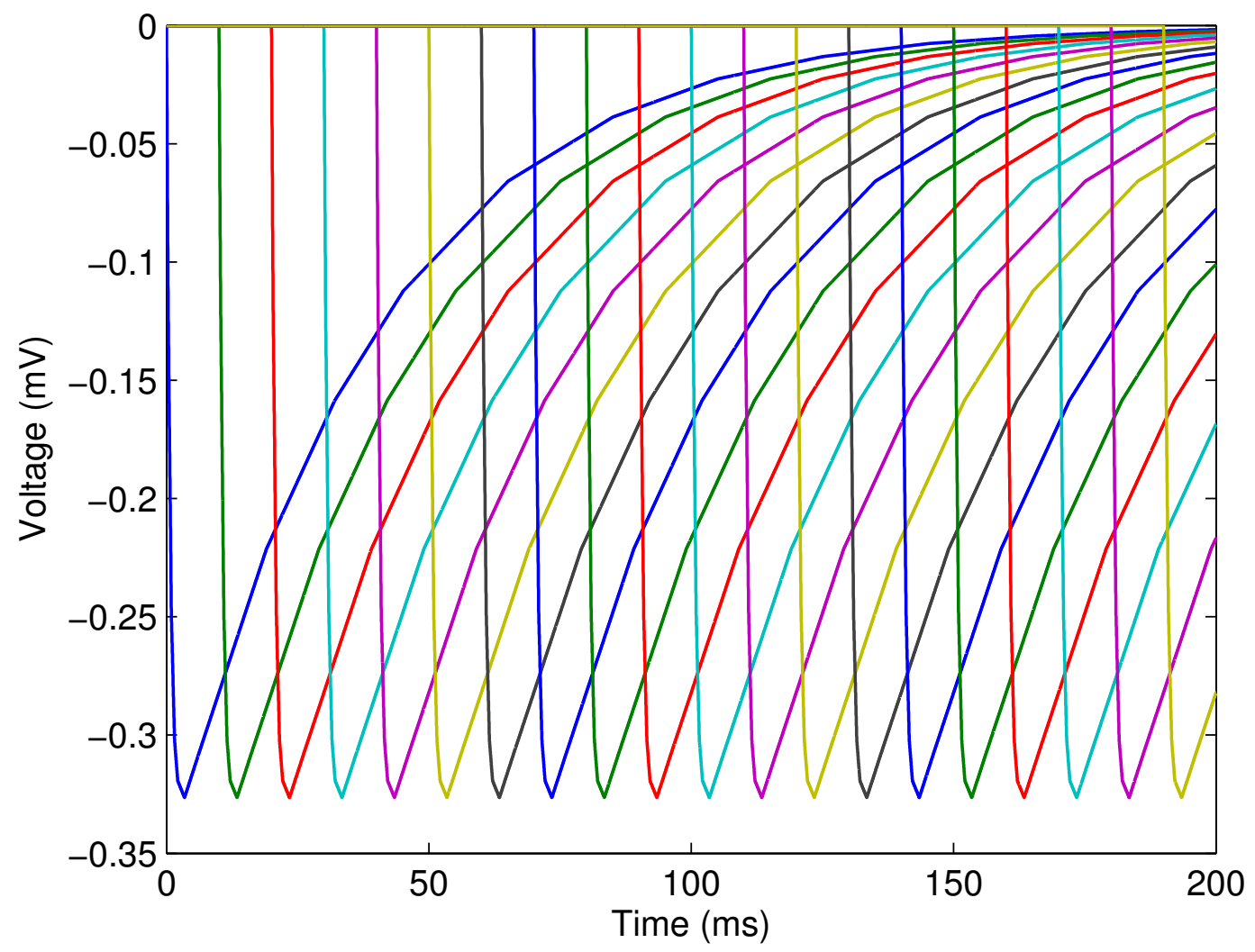

Figure 4.9: Basis functions for asynchronous presynaptic firing of $\mathrm{GABA}_{\mathrm{A}}$, slowmediated neurons, $\boldsymbol{\Lambda}_{\mathbf{n}}=v_{S}\left(t-t_{n}\right)$ for $n=1, \ldots, 20$ 


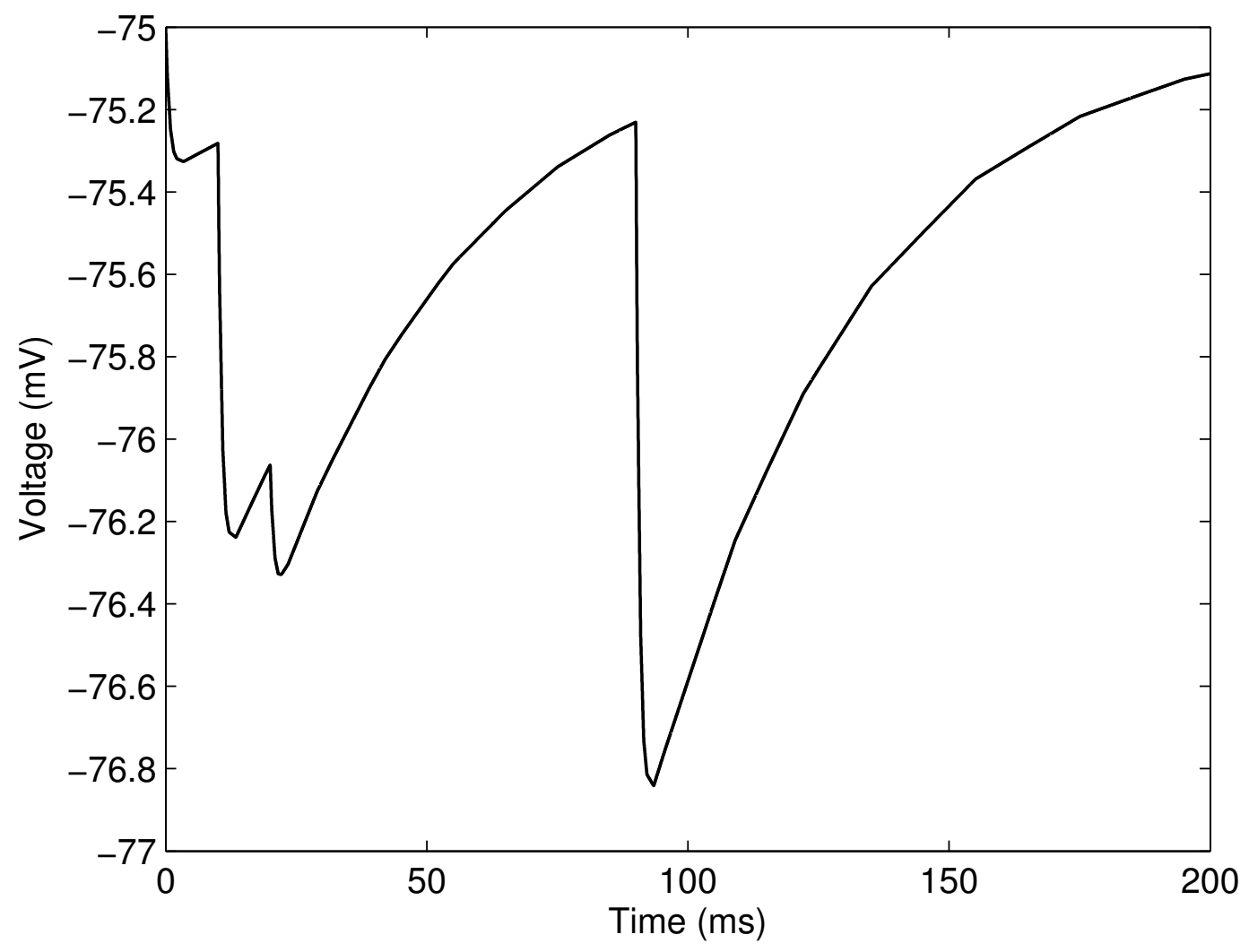

Figure 4.10: Compound function $\Phi_{G A B A, \text { slow }}(t)+V_{m}$

Using Szlavik's algorithm to decompose the signal with $\boldsymbol{\Psi}=\Phi_{G A B A, \text { slow }}(t)$ and $\boldsymbol{\Lambda}_{\mathbf{n}}=$ $v_{S}\left(t-t_{n}\right)$ for $n=1, \ldots, 20$ yielded coefficients $\beta_{1}^{\prime}=1, \beta_{2}^{\prime}=3, \beta_{3}^{\prime}=1$, and $\beta_{10}^{\prime}=5$, and all other coefficients equal to zero. The algorithm again yielded an exact estimation of the frequency of each postsynaptic potential in the compound potential. A histogram of the frequencies can be seen in Figure 4.11. 


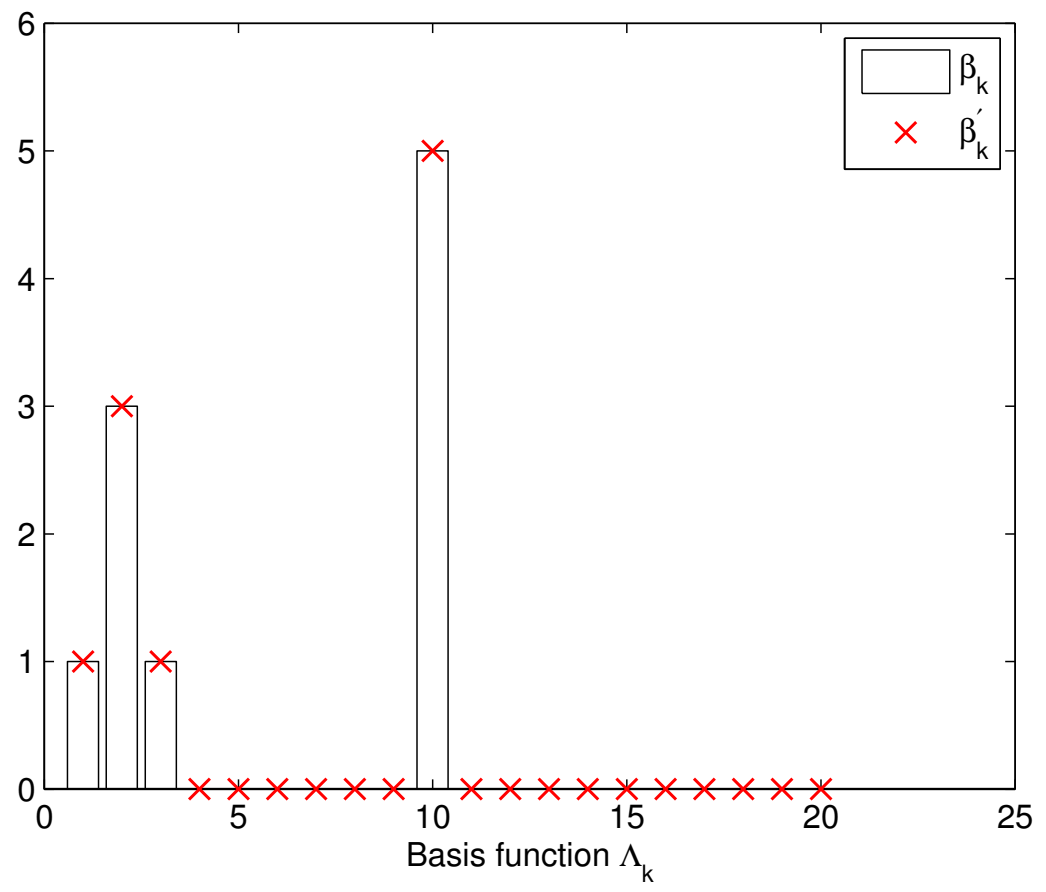

Figure 4.11: Frequency distribution from decomposition of $\Phi_{G A B A, \text { slow }}(t)$.

The compound potential $\Phi_{G A B A, \text { slow }}(t)$ was decomposed using the generalized Fourier series for comparison. As seen in Figure 4.12, the generalized Fourier series gave very incorrect estimates since the basis functions overlap so much. 


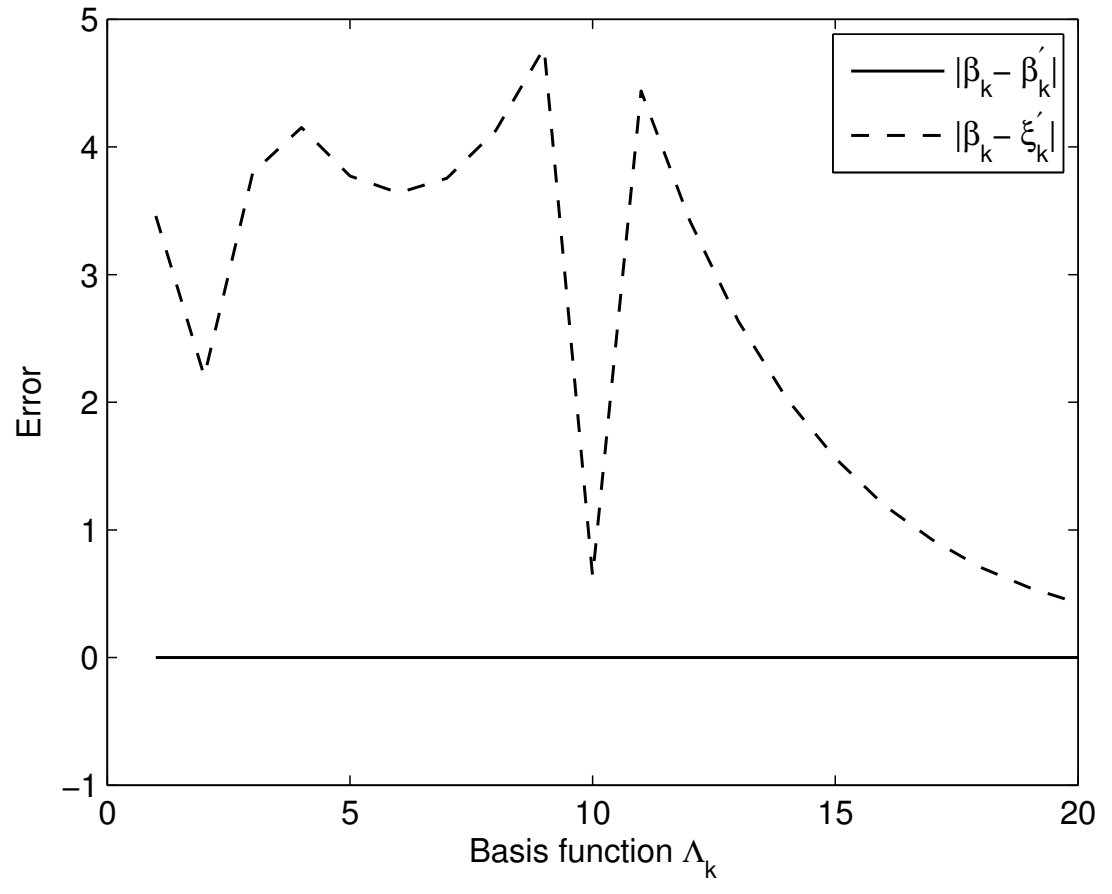

Figure 4.12: Comparison of error from generalized Fourier series approach to Szlavik perturbation decomposition approach for $\mathrm{GABA}_{\mathrm{A}}$, slow-mediated potentials.

\subsection{3 $\quad$ GABA $_{\mathrm{A}, \text { fast-mediated potentials }}$}

An arbitrary compound potential $\Phi_{G A B A, f a s t}(t)$ was defined as follows:

$$
\begin{aligned}
\Phi_{G A B A, f a s t}(t) & =5 v_{F}\left(t-t_{2}\right)+3 v_{F}\left(t-t_{13}\right)+v_{F}\left(t-t_{14}\right)+3 v_{F}\left(t-t_{15}\right) \\
t_{n} & =(n-1) \cdot 10 \mathrm{~ms}
\end{aligned}
$$

The basis functions $v_{F}\left(t-t_{1}\right), \ldots, v_{F}\left(t-t_{20}\right)$ and the compound function $\Phi_{G A B A, \text { fast }}(t)$ can be seen in Figures 4.13 and 4.14, respectively. 


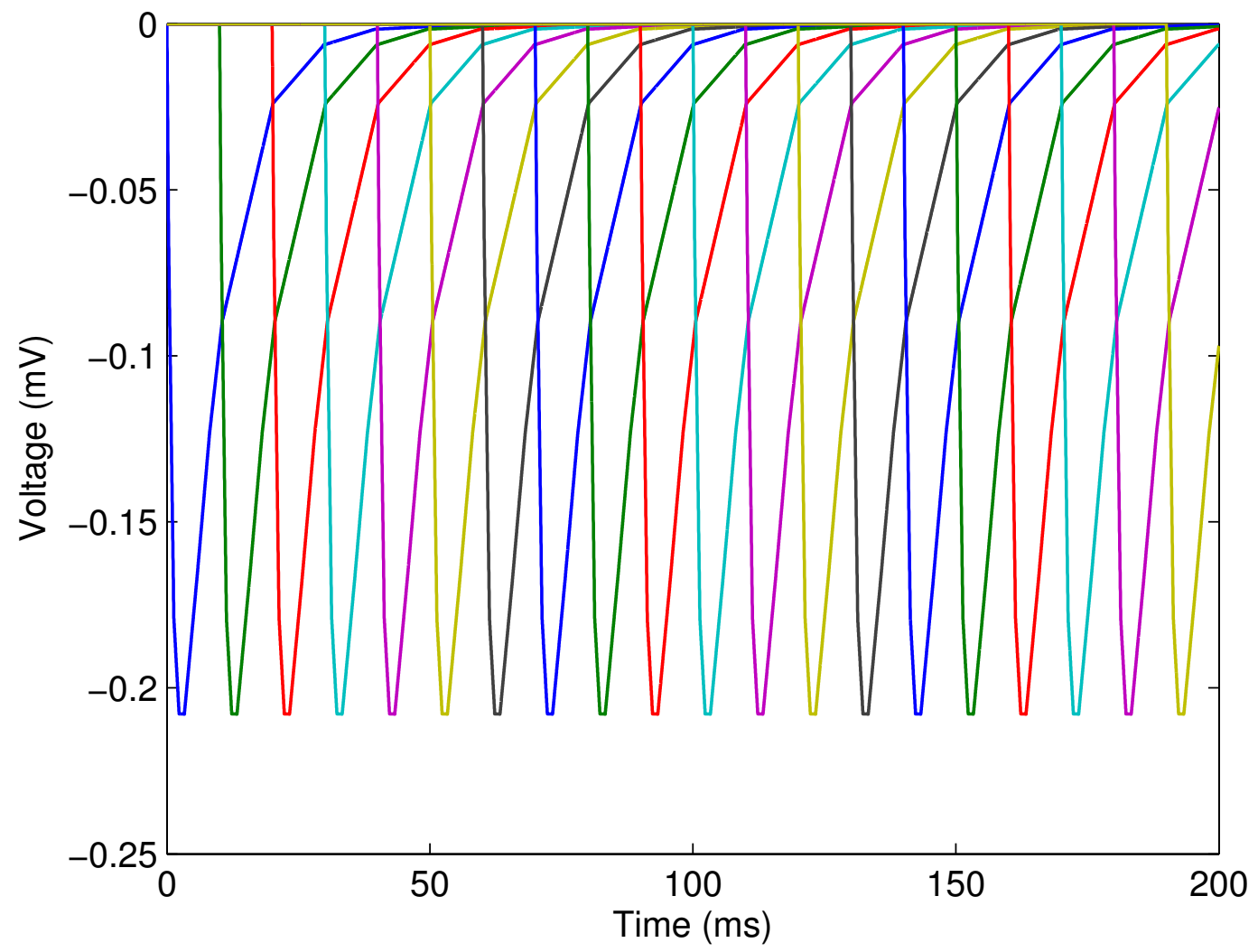

Figure 4.13: Basis functions for asynchronous presynaptic firing of $\mathrm{GABA}_{\mathrm{A}}$, fastmediated neurons, $\boldsymbol{\Lambda}_{\mathbf{n}}=v_{F}\left(t-t_{n}\right)$ for $n=1, \ldots, 20$ 


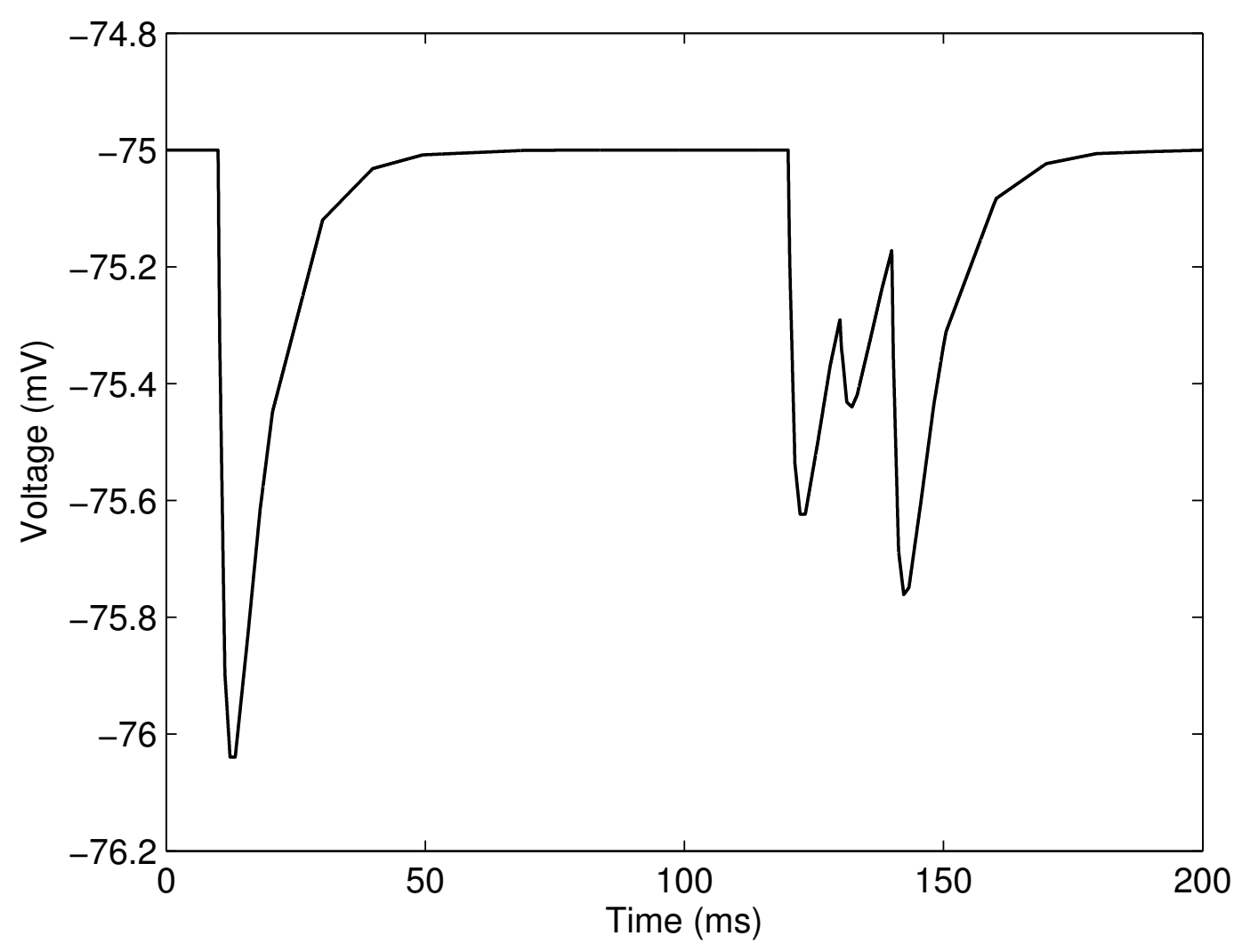

Figure 4.14: Compound function $\Phi_{G A B A, f a s t}(t)+V_{m}$

Using Szlavik's algorithm to decompose the signal with $\boldsymbol{\Psi}=\Phi_{G A B A, f a s t}(t)$ and $\boldsymbol{\Lambda}_{\mathbf{n}}=$ $v_{F}\left(t-t_{n}\right)$ for $n=1, \ldots, 20$ yielded coefficients $\beta_{2}^{\prime}=5, \beta_{13}^{\prime}=3, \beta_{14}^{\prime}=1$, and $\beta_{15}^{\prime}=3$, and all other coefficients equal to zero. The algorithm again yielded an exact estimation of the number of each repolarization in the compound potential. A histogram of the frequencies can be seen in Figure 4.15. 


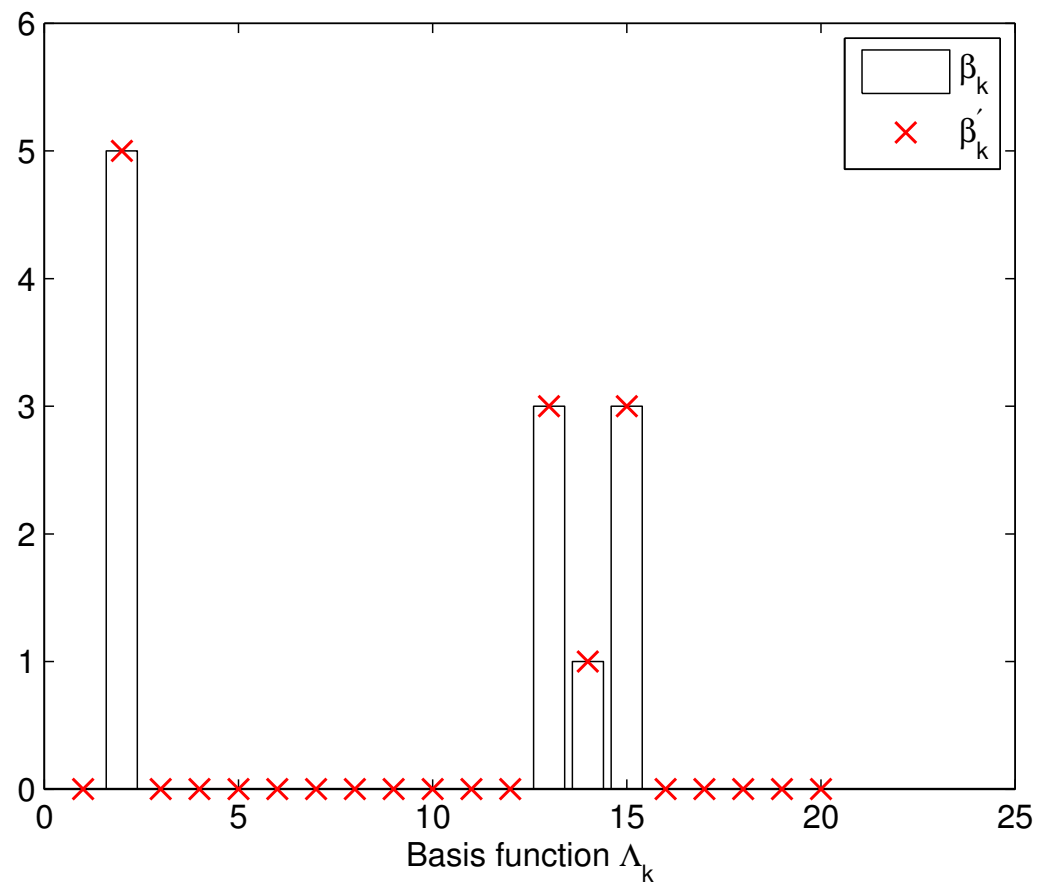

Figure 4.15: Frequency distribution from decomposition of $\Phi_{G A B A, f a s t}(t)$.

The compound potential $\Phi_{G A B A, f a s t}(t)$ was decomposed using the generalized Fourier series for comparison. As seen in Figure 4.16, the generalized Fourier series once again gave incorrect estimates. 


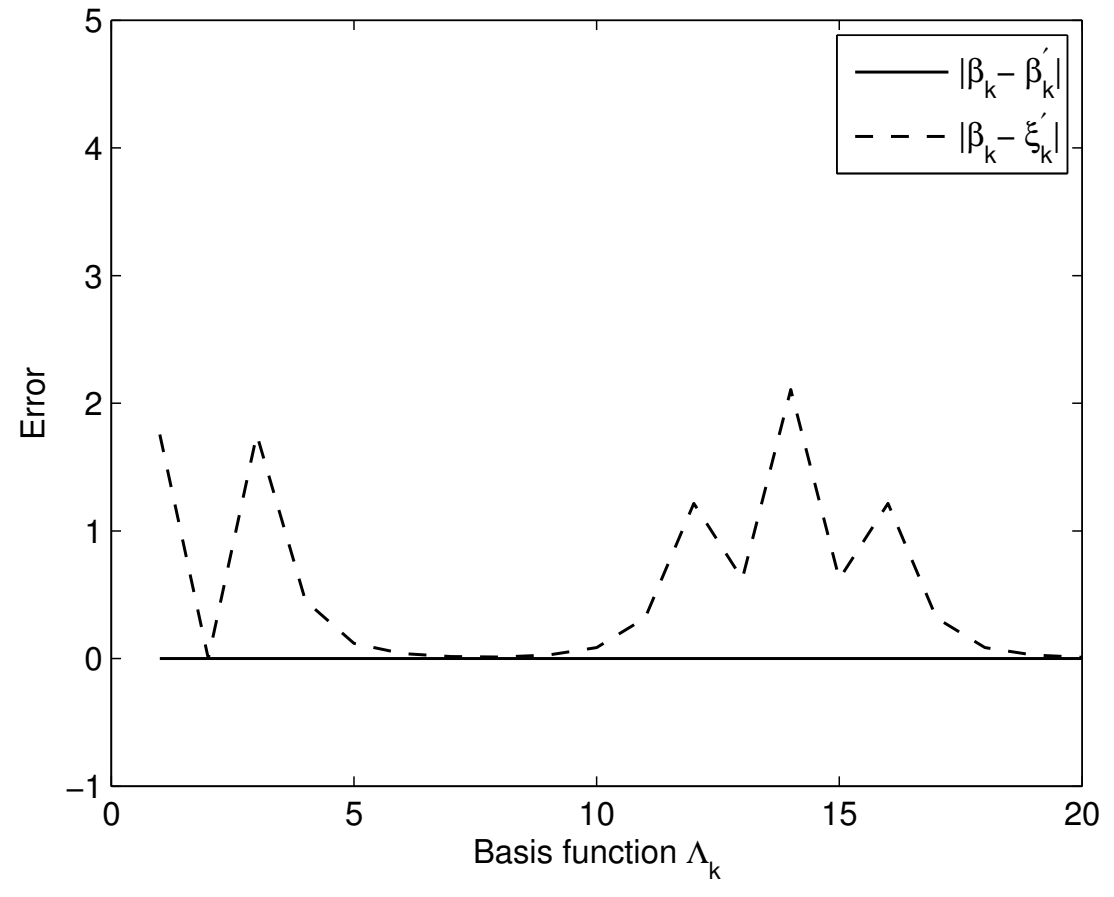

Figure 4.16: Comparison of error from generalized Fourier series approach to Szlavik perturbation decomposition approach for $\mathrm{GABA}_{\mathrm{A}}$, fast-mediated potentials.

\subsubsection{NMDA-mediated potentials}

An arbitrary compound potential $\Phi_{N M D A}(t)$ was defined as follows:

$$
\begin{aligned}
\Phi_{N M D A}(t) & =3 v_{N}\left(t-t_{3}\right)+v_{N}\left(t-t_{6}\right)+v_{N}\left(t-t_{18}\right)+3 v_{N}\left(t-t_{19}\right)+v_{N}\left(t-t_{20}\right) \\
t_{n} & =(n-1) \cdot 10 \mathrm{~ms}
\end{aligned}
$$

The basis functions $v_{N}\left(t-t_{1}\right), \ldots, v_{N}\left(t-t_{20}\right)$ and the compound function $\Phi_{N M D A}(t)$ can be seen in Figures 4.17 and 4.18, respectively. 


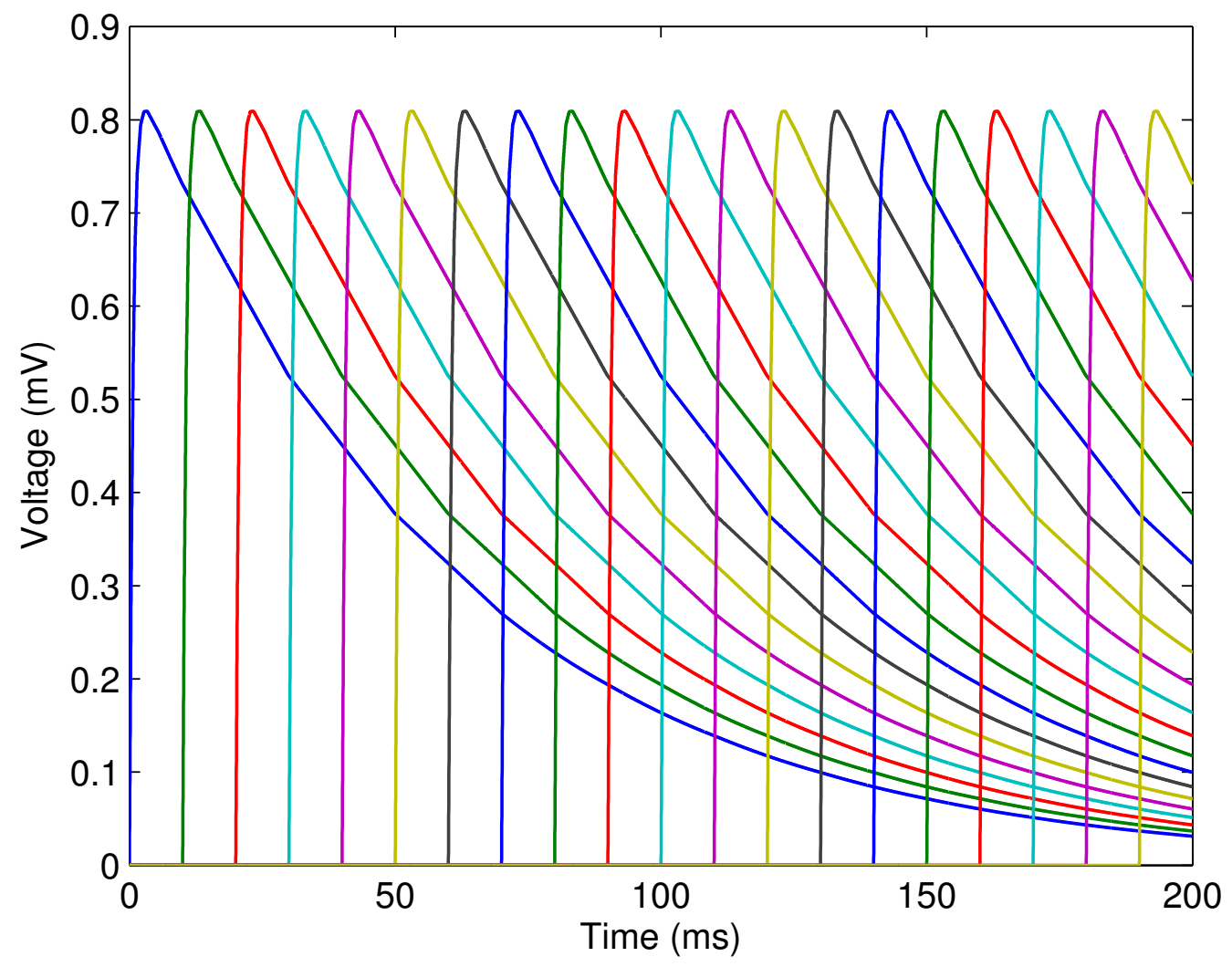

Figure 4.17: Basis functions for asynchronous presynaptic firing of NMDAmediated neurons, $\boldsymbol{\Lambda}_{\mathbf{n}}=v_{N}\left(t-t_{n}\right)$ for $n=1, \ldots, 20$ 


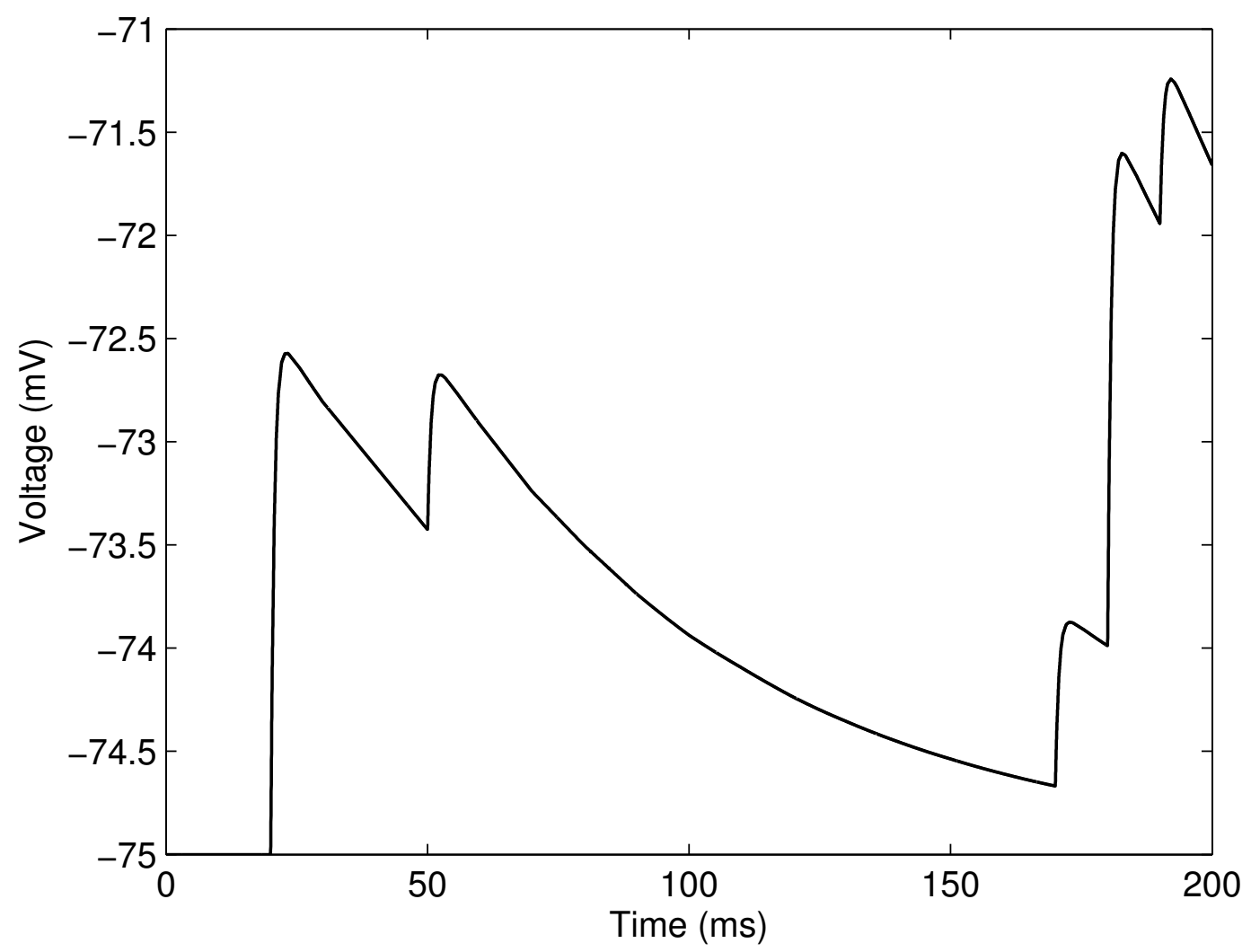

Figure 4.18: Compound function $\Phi_{N M D A}(t)+V_{m}$

Using Szlavik's algorithm to decompose the signal with $\boldsymbol{\Psi}=\Phi_{N M D A}(t)$ and $\boldsymbol{\Lambda}_{\mathbf{n}}=v_{N}(t-$ $\left.t_{n}\right)$ for $n=1, \ldots, 20$ yielded coefficients $\beta_{3}^{\prime}=3, \beta_{6}^{\prime}=1, \beta_{18}^{\prime}=1, \beta_{19}^{\prime}=3$, and $\beta_{20}^{\prime}=1$, and all other coefficients equal to zero. The algorithm again yielded an exact estimation of the number of each depolarization in the compound potential. A histogram of the frequencies can be seen in Figure 4.19. 


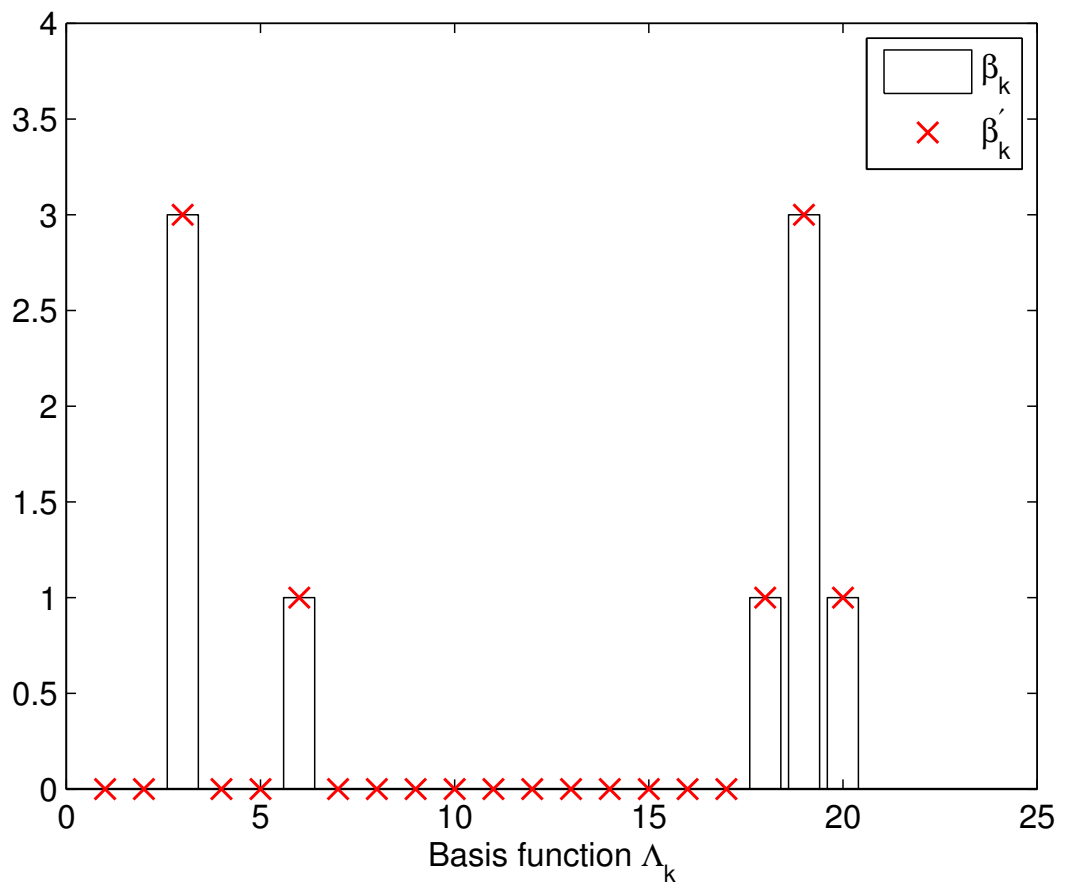

Figure 4.19: Frequency distribution from decomposition of $\Phi_{N M D A}(t)$.

The compound potential $\Phi_{N M D A}(t)$ was decomposed using the generalized Fourier series for comparison. As seen in Figure 4.20, the generalized Fourier series once again gave incorrect estimates. 


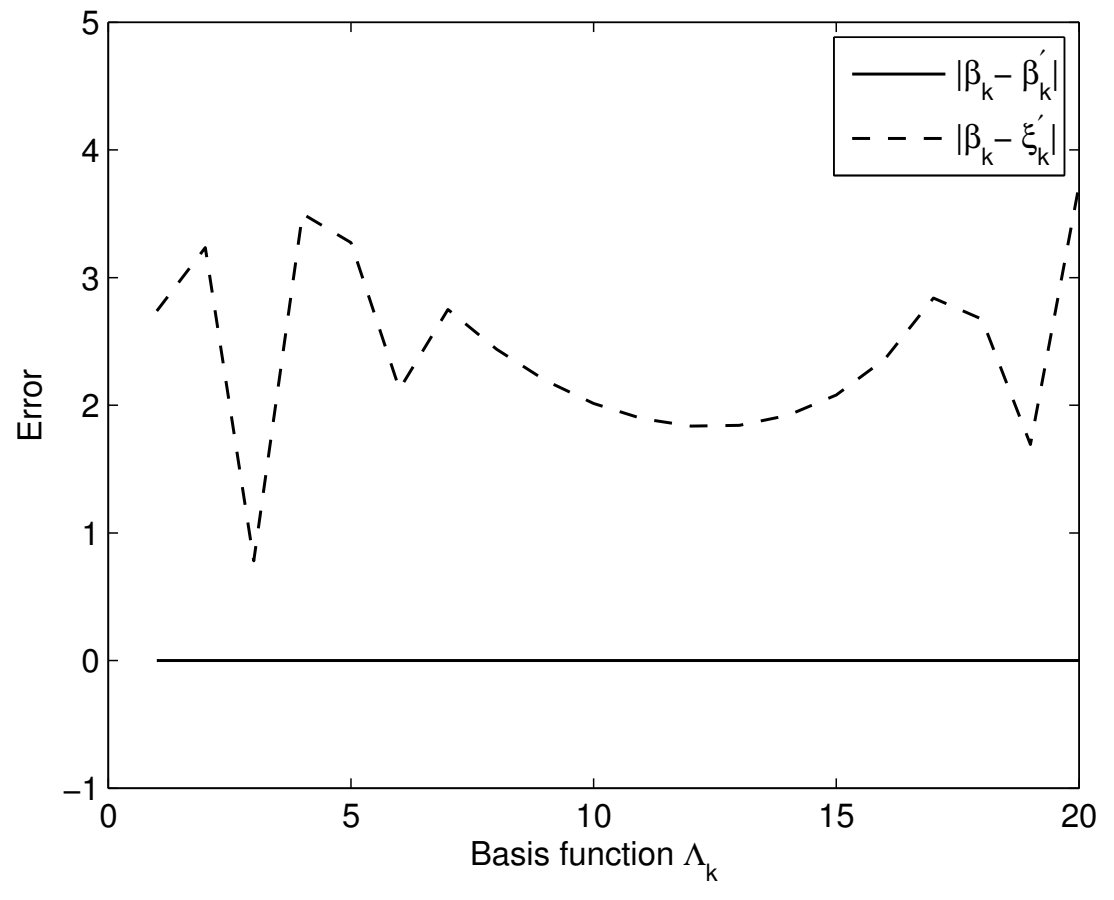

Figure 4.20: Comparison of error from generalized Fourier series approach to Szlavik perturbation decomposition approach for NMDA-mediated potentials.

\subsection{Experimental validation}

In order to fully evaluate the perturbation decomposition technique for this application, it was tested against experimental data. Abrahamsson et al. did a study on synaptic integration of cerebellar interneurons in which dendrite and somatic neurons were stimulated with spike trains [29]. Figure 4.21(a) shows dendritic and somatic responses to a $50 \mathrm{~Hz}$ train applied by electrical stimulation. The dendritic response (gray) was traced using MATLAB and stored in a vector $\boldsymbol{\Psi}$. Next, it was hypothesized that these were NMDA-mediated depolarizations based on the decay rates. A basis potential $v_{N}(t)$ was generated by using the physiological values in Table 4.1 to tune to the traced data. The algorithm was then used with $\boldsymbol{\Lambda}_{\mathbf{n}}=v_{N}\left(t-t_{n}\right)$ for $n=1, \ldots, 50$ where $t_{n}=(n-1) \cdot 1.2 \mathrm{~ms}$. 


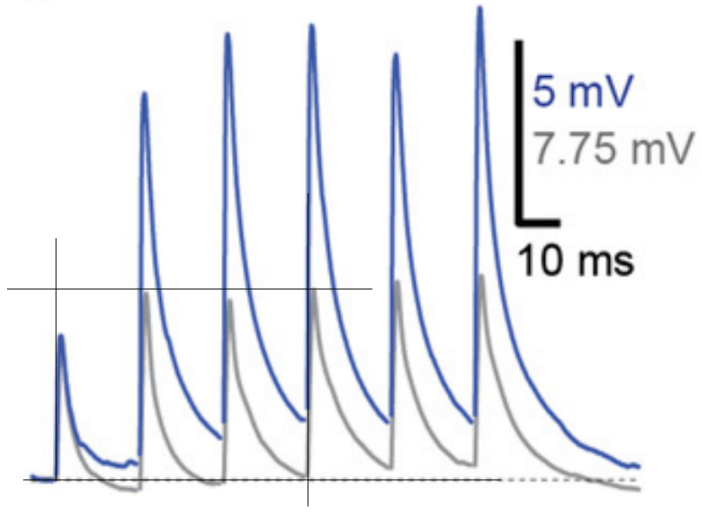

(a) Dendritic (gray) and somatic (blue) EPSPs in response to a $50 \mathrm{~Hz}$ train [29].

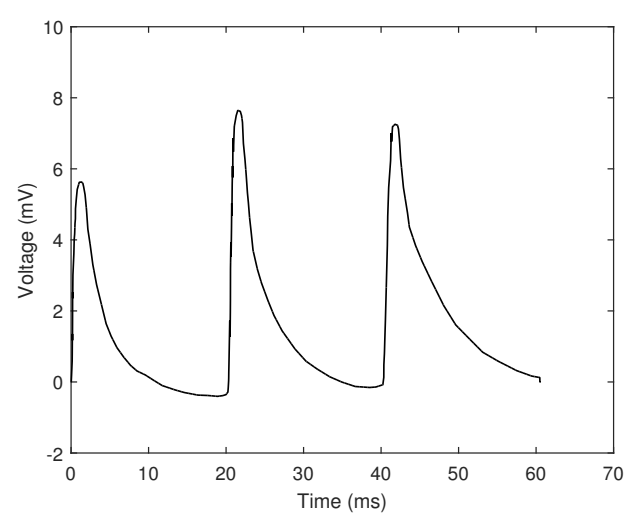

(b) Data trace of three dendritic spikes from (a) using MATLAB.

Figure 4.21: Data trace of compound AMPA PSP.

Table 4.1: Changes to AMPA PSP values for curve fitting.

\begin{tabular}{lll}
\hline Term & Value & Description \\
\hline$\tau_{A}$ & $4 \mathrm{~ms}$ & AMPA time constant \\
$\overline{g_{A}}$ & $3.5 \mathrm{nS}$ & AMPA max synaptic conductance
\end{tabular}




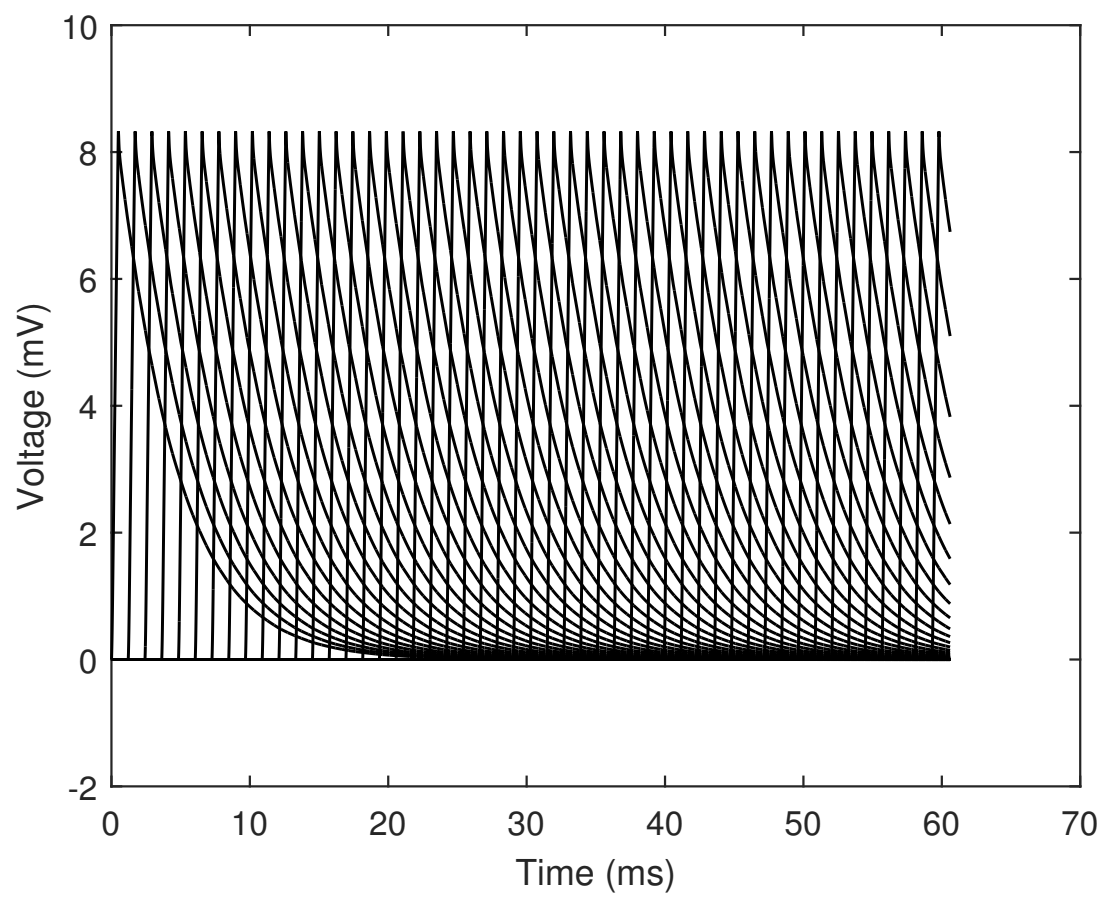

Figure 4.22: Basis functions for decomposition of traced data from Abrahamsson et al.

Szlavik's technique returned the frequency distribution $\mathbf{f}$ depicted in Figure 4.23. It clearly has three distinct peaks as expected. 


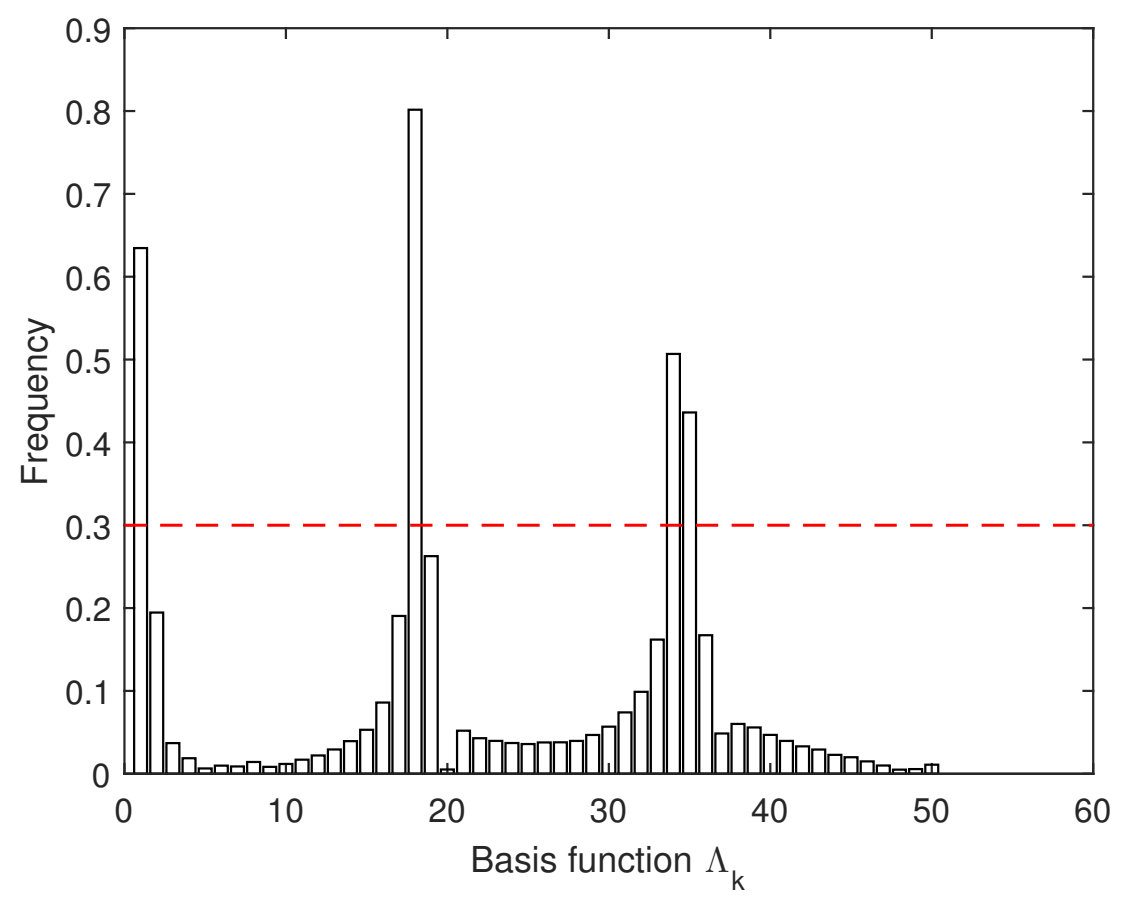

Figure 4.23: Frequency plot of Perturbation Decomposition Method on experimental data. Dashed red line denotes chosen cutoff frequency, $f_{c}=0.3$.

A raw reconstruction of the signal, $\mathbf{f} \cdot \boldsymbol{\Psi}$ can be seen in Figure 4.24(a). To get a better reconstruction of the signal, a discrete high pass filter with a cutoff frequency of $f_{c}=0.3$ was applied to the frequency distribution $\mathbf{f}$ to create the distribution $\mathbf{g}$. The $i$-th element of $\mathrm{g}$ is

$$
\mathbf{g}_{i}=\left\{\begin{array}{ll}
\mathbf{f}_{i} & \text { for } \mathbf{f}_{i}>0.3 \\
0 & \text { for } \mathbf{f}_{i} \leq 0.3
\end{array} .\right.
$$

This filter, depicted as a dashed red line in Figure 4.23, greatly reduces the error of the reconstruction and gives a satisfactory estimate of the original signal. Whereas the raw reconstruction $\mathbf{f} \cdot \boldsymbol{\Psi}$ fits the data with $R^{2}=0.43$, the filtered reconstruction $\mathbf{g} \cdot \boldsymbol{\Psi}$ fits the data with $R^{2}=0.89$. This reconstruction $\mathbf{g} \cdot \boldsymbol{\Psi}$ can be seen in Figure 4.24(b). 


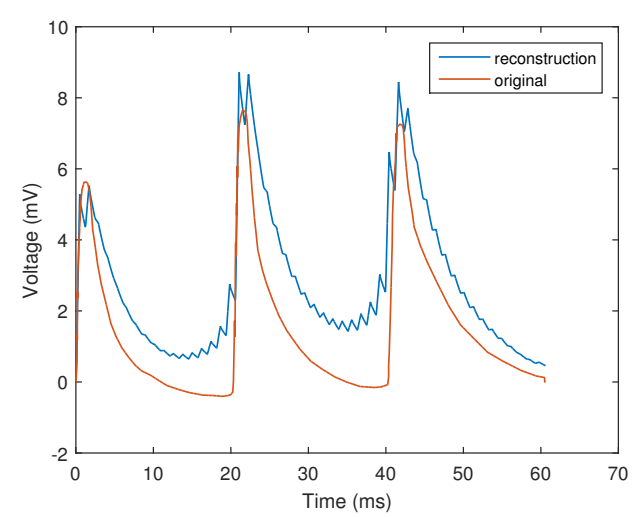

(a) Raw reconstruction

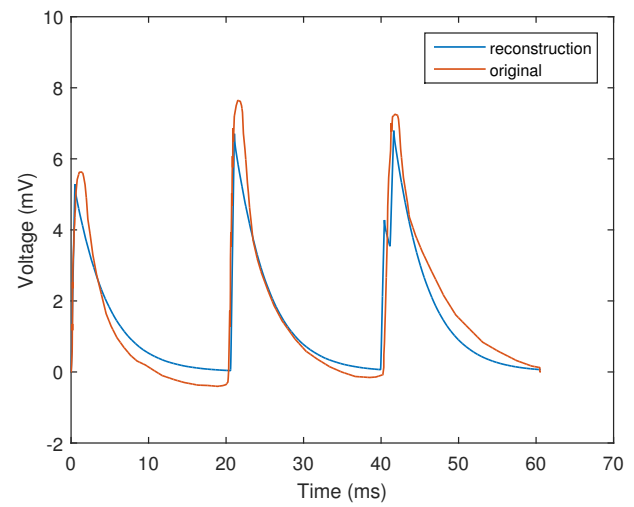

(b) High-pass filtered reconstruction $\left(f_{c}=0.3\right)$

Figure 4.24: Reconstruction of EPSP spike train from Abrahamsson et al. study $[29]$.

The raw frequency distribution, $\mathbf{f}=\left[\beta_{1}^{\prime}, \ldots, \beta_{n}^{\prime}\right]$, was then compared to the frequency distribution returned by the generalized Fourier series distribution, $\mathbf{h}=\left[\xi_{1}^{\prime}, \ldots, \xi_{n}^{\prime}\right]$. As illustrated in Figure 4.25, Szlavik's method gave much more distinct peaks, and thus a better estimation of the compound postsynaptic potential.

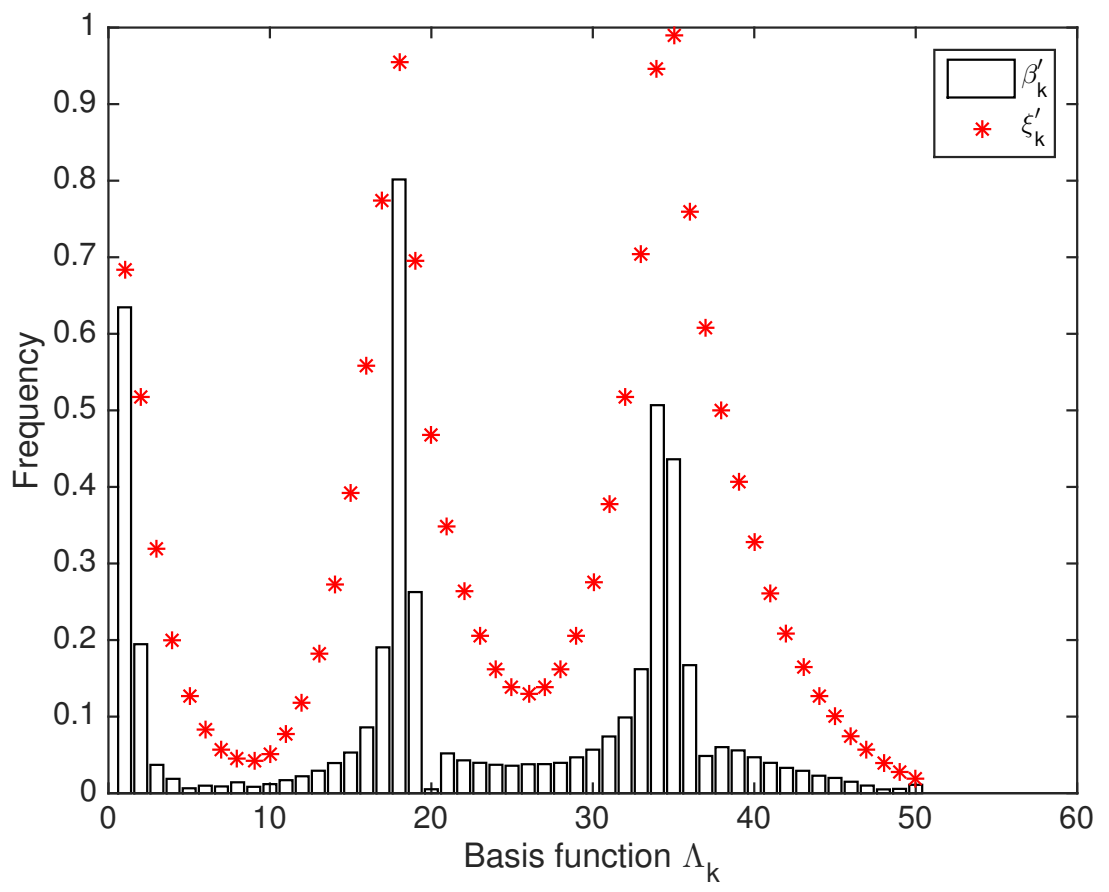

Figure 4.25: Comparison of Szlavik perturbation decomposition estimations $\left(\beta_{k}^{\prime}\right)$ to generalized Fourier series estimations $\left(\xi_{k}^{\prime}\right)$ on experimental data from Abrahamsson et al. study. 


\section{Discussion}

The results of this work have shown not only that Szlavik's perturbation decomposition method is a viable method for analyzing postsynaptic potentials, but also that it can be used as a substitute for the generalized Fourier series when working with non-orthogonal functions. The perturbation decomposition method successfully decomposed compound functions of time-shifted AMPA receptor-mediated potentials, time-shifted $\mathrm{GABA}_{\mathrm{A} \text {, slow }}$ receptormediated potentials, time-shifted $\mathrm{GABA}_{\mathrm{A}}$, fast receptor-mediated potentials, time-shifted NMDA receptor-mediated potentials, as well as compound potentials from all four types of receptors. In contrast, the generalized Fourier series method only gave close estimates for the AMPA receptor-mediated potentials. In addition, the perturbation decomposition method was evaluated by applying it to experimental data, yielding satisfactory results.

\subsection{Implications}

The experimental validation demonstrated that the perturbation decomposition method can be applied to whole patch-clamp recordings to estimate the contribution of different receptors to a neuron's postsynaptic potential signal. The output of this decomposition could be used to study the pathogenesis of disorders such as Parkinson's and Alzheimer's by quanti-

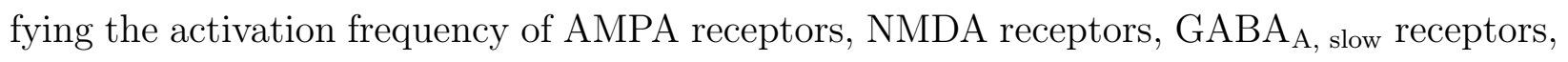
and $\mathrm{GABA}_{\mathrm{A}}$, fast receptors in healthy and diseased neurons. Automated whole-cell patch clamping of rats in vivo is possible using a robot developed by Kodandaramaiah et al. [30]. If such a robot can be created for use with humans, the perturbation decomposition method could be used as a technique for diagnosis of neurological and mental disorders. Having a quantitative test for mental disorders could help reduce the large number of mental disorder misdiagnoses.

This research, along with Szlavik's work [28] forms the basis for a general mathematical 
method of biopotential signal analysis. Given a signal, such as a compound postsynaptic potential reading from a neuron, not much analysis can be done directly due to the complexity of the signal. However, by introducing known models, such as the time responses of singlereceptor postsynaptic potentials, the compound signal can be analyzed and studied effectively using Szlavik's perturbation decomposition method. Thus, this technique can be applied to any problem where the signal of interest is a combination of other signals that can be accurately modelled.

\subsection{Limitations and Future Work}

Due to memory constraints, this method could not be used to decompose a compound signal into time-shifted potentials from all four different types of neurotransmitter receptors. However, given more computing resources, this decomposition could be accomplished. In

addition, future work can be undertaken to account for spatial effects and non-linearity in postsynaptic potential summation. One such non-linearity is the NMDA receptor's $\mathrm{Mg}^{2+}$ block mechanism, which makes the receptor both ligand-gated and voltage-gated. This block mechanism was assumed to be negligible, but should be incorporated in future iterations.

To apply this method to neurological diagnostics, research should be done to determine the receptor activation frequency of healthy and diseased neurons for various disorders. Other PSP models such as quantal PSP models could be used as basis functions to estimate neurotransmitter concentration in the synaptic cleft during transmission. Moving outside the application of postsynaptic potentials, Szlavik's perturbation method could be applied to the inverse problem in EEG source localization, which is the problem of predicting the current sources that caused a particular voltage reading on the scalp [31].

\subsection{Conclusion}

Many brain disorders manifest themselves at a small scale. Often, neurological disorders are caused by small changes in the amount or frequency of activation of neurotransmitter receptors in neurons. If these changes can be detected, the associated disorders could be more effectively studied or diagnosed. The perturbation decomposition technique developed by R. Szlavik can be used as a tool to estimate the receptor activation frequency of a given 
neuron, assuming the postsynaptic potential models accurately model the electrical activity during the neurotransmission events for each receptor. If an in vivo patch clamp machine can be made for use with humans, this could change the way many brain disorders are diagnosed. 


\section{BIBLIOGRAPHY}

[1] "Cal Poly Github." http://www.github.com/CalPoly.

[2] J. H. Kaas, "The evolution of the complex sensory and motor systems of the human brain," Brain Research Bulletin, vol. 75, no. 24, pp. 384 - 390, 2008. Evolution and the Generation of Novelties in the Nervous System Proceedings of the 5th European Conference on Comparative Neurobiology: April 25-28, 2007, Musum National dHistoire Naturelle, Paris.

[3] D. A. Drachman, "Do we have brain to spare?," Neurology, vol. 64, no. 12, pp. 2004-2005, 2005.

[4] S. H. Snyder and C. D. Ferris, "Novel neurotransmitters and their neuropsychiatric relevance," American Journal of Psychiatry, vol. 157, no. 11, pp. 1738-1751, 2000. PMID: 11058466.

[5] Y. Xu, J. Yan, P. Zhou, J. Li, H. Gao, Y. Xia, and Q. Wang, "Neurotransmitter receptors and cognitive dysfunction in alzheimer's disease and parkinson's disease," Progress in Neurobiology, vol. 97, no. 1, pp. 1 - 13, 2012.

[6] R. Jones, "From amyloid- $\beta$ to receptor endocytosis," Nat Rev Neurosci, vol. 6, no. 9, pp. 669-668, 2005.

[7] D. Ulrich, "Amyloid- $\beta$ impairs synaptic inhibition via GABA(A) receptor endocytosis.," The Journal of Neuroscience, vol. 35, no. 5, pp. 9205-10, 2015.

[8] A. Scimemi and M. Beato, "Determining the neurotransmitter concentration profile at active synapses," Mol Neurobiol, vol. 40, no. 3, pp. 289-306, 2009.

[9] K. Abe and H. Saito, "Pharmacological isolation and characterization of NMDA receptor-mediated synaptic potential in the dentate gyrus of rat hippocampal slices," The Japanese Journal of Pharmacology, vol. 61, no. 4, pp. 333-340, 1993.

[10] B. J. Vizcarra-Chacón, M. A. Arias-García, M. B. Pérez-Ramírez, E. Flores-Barrera, D. Tapia, R. Drucker-Colin, J. Bargas, and E. Galarraga, "Contribution of different classes of glutamate receptors in the corticostriatal polysynaptic responses from 
striatal direct and indirect projection neurons," BMC Neuroscience, vol. 14, no. 1, pp. 1-17, 2013.

[11] S. H. Wu, C. L. Ma, and J. B. Kelly, "Contribution of AMPA, NMDA, and GABA-A receptors to temporal pattern of postsynaptic responses in the inferior colliculus of the rat," J. Neurosci., vol. 24, no. 19, pp. 4625-4634, 2004.

[12] E. N. Marieb and K. Hoehn, Human Anatomy and Physiology. Pearson, 10th ed., 2015.

[13] R. Webster, Neurotransmitter Systems and Function: Overview, pp. 1-32. John Wiley \& Sons, Ltd, 2002.

[14] D. Purves, D. Fitzpatrick, L. Katz, A. Lamantia, J. McNamara, S. Williams, and G. Augustine, Neuroscience. Sinauer Associates, 2001.

[15] P. J. Magistretti, L. Pellerin, D. L. Rothman, and R. G. Shulman, "Energy on demand," Science, vol. 283, no. 5401, p. 496, 1999.

[16] D. F. Schafer and E. A. Jones, "Hepatic encephalopathy and the -aminobutyric-acid neurotransmitter system," The Lancet, vol. 319, no. 8262, pp. 18 - 20, 1982.

[17] W. J. McEntee and T. H. Crook, "Glutamate: its role in learning, memory, and the aging brain," Psychopharmacology, vol. 111, no. 4, pp. 391-401.

[18] F. Fonnum, "Glutamate: A neurotransmitter in mammalian brain," Journal of Neurochemistry, vol. 42, no. 1, pp. 1-11, 1984.

[19] A. V. Kalueff and D. J. Nutt, "Role of GABA in anxiety and depression," Depression and Anxiety, vol. 24, no. 7, pp. 495-517, 2007.

[20] R. Malinow and R. C. Malenka, "AMPA receptor trafficking and synaptic plasticity," Annual Review of Neuroscience, vol. 25, no. 1, pp. 103-126, 2002. PMID: 12052905.

[21] H. Meguro, H. Mori, K. Araki, E. Kushiya, T. Kutsuwada, M. Yamazaki, T. Kumanishi, M. Arakawa, K. Sakimura, and M. Mishina, "Functional characterization of a heteromeric NMDA receptor channel expressed from cloned cDNAs," Nature, vol. 357, no. 6373, pp. 70-74, 1992. 
[22] J. Dalmau, A. J. Gleichman, E. G. Hughes, J. E. Rossi, X. Peng, M. Lai, S. K. Dessain, M. R. Rosenfeld, R. Balice-Gordon, and D. R. Lynch, "Anti-NMDA-receptor encephalitis: case series and analysis of the effects of antibodies," The Lancet Neurology, vol. 7, no. 12, pp. 1091 - 1098, 2008.

[23] C. G. Parsons, A. Stöffler, and W. Danysz, "Memantine: a NMDA receptor antagonist that improves memory by restoration of homeostasis in the glutamatergic system - too little activation is bad, too much is even worse," Neuropharmacology, vol. 53, no. 6, pp. $699-723,2007$.

[24] B. Lüscher and C. A. Keller, "Regulation of GABA-A receptor trafficking, channel activity, and functional plasticity of inhibitory synapses," Pharmacology $\&$ Therapeutics, vol. 102, no. 3, pp. 195 - 221, 2004.

[25] M. I. Banks, T. B. Li, and R. A. Pearce, "The synaptic basis of GABA-A,slow.," J Neurosci, vol. 18, no. 4, pp. 1305-1317, 1998.

[26] L. Zhou, S. Zhao, and F. Nadim, "Neuromodulation of short-term synaptic dynamics examined in a mechanistic model based on kinetics of calcium currents," Neurocomputing, vol. 70, no. 10-12, pp. 2050-2054, 2007.

[27] A. Kapur, W. W. Lytton, K. L. Ketchum, and L. B. Haberly, "Regulation of the NMDA component of EPSPs by different components of postsynaptic GABAergic inhibition: computer simulation analysis in piriform cortex," J Neurophysiol, vol. 78, no. 5, pp. 2546-59, 1997.

[28] R. B. Szlavik, "A perturbation based decomposition of compound-evoked potentials for characterization of nerve fiber size distributions," IEEE Transactions on Neural Systems and Rehabilitation Engineering, vol. 24, no. 2, pp. 212-216, 2016.

[29] T. Abrahamsson, L. Cathala, K. Matsui, R. Shigemoto, and D. A. DiGregorio, "Thin dendrites of cerebellar interneurons confer sublinear synaptic integration and a gradient of short-term plasticity," Neuron, vol. 73, no. 6, pp. 1159 - 1172, 2012.

[30] S. B. Kodandaramaiah, G. T. Franzesi, B. Y. Chow, E. S. Boyden, and C. R. Forest, "Automated whole-cell patch clamp electrophysiology of neurons in vivo," Nat Methods, vol. 9, pp. 585-587, Jun 2012. 22561988[pmid]. 
[31] R. Grech, T. Cassar, J. Muscat, K. P. Camilleri, S. G. Fabri, M. Zervakis, P. Xanthopoulos, V. Sakkalis, and B. Vanrumste, "Review on solving the inverse problem in eeg source analysis," Journal of NeuroEngineering and Rehabilitation, vol. 5, no. 1, pp. 1-33, 2008. 


\section{APPENDIX A}

\section{A.1 PostsynapticPotential.m}

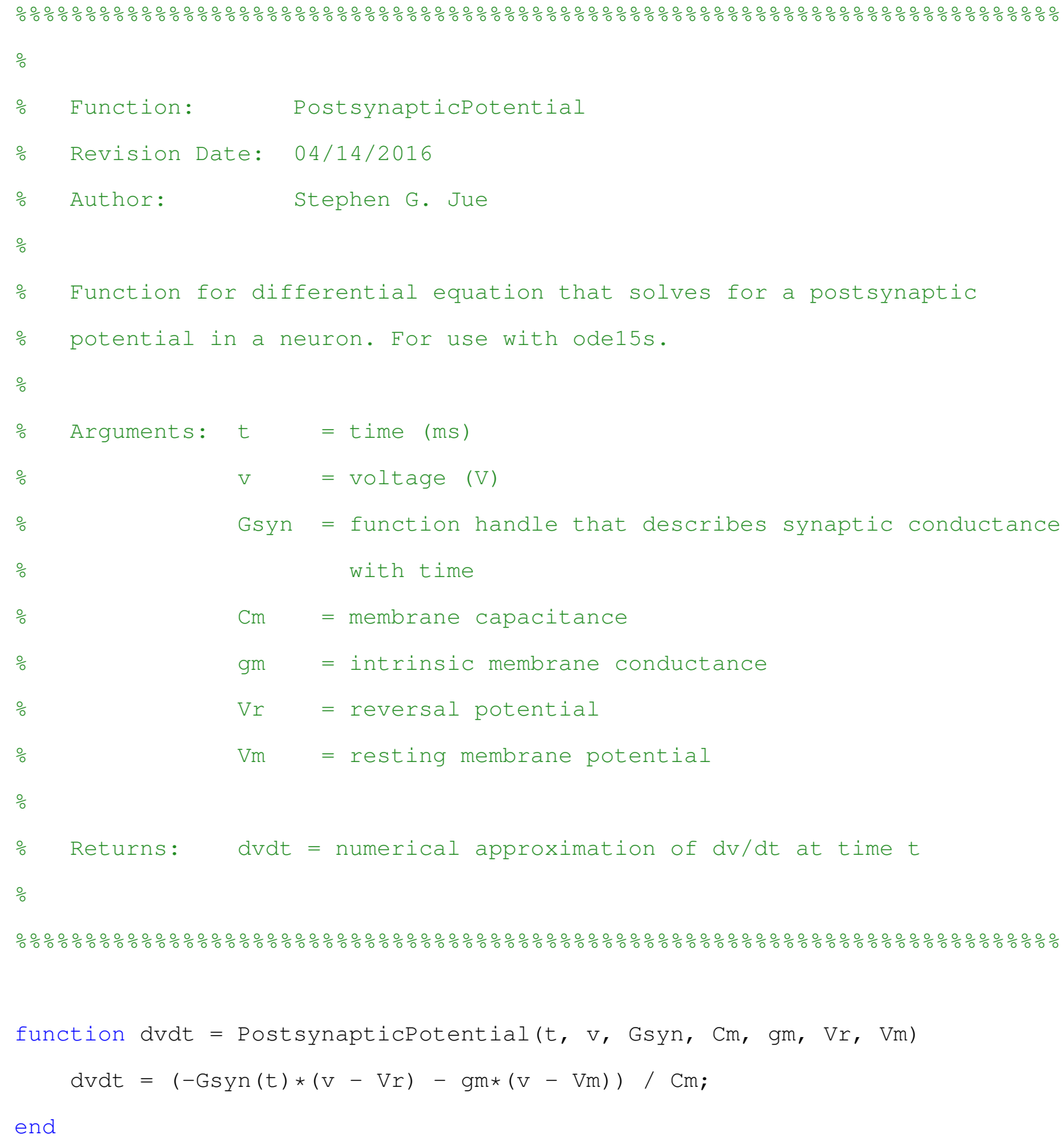




\section{A.2 VectorBasis.m}

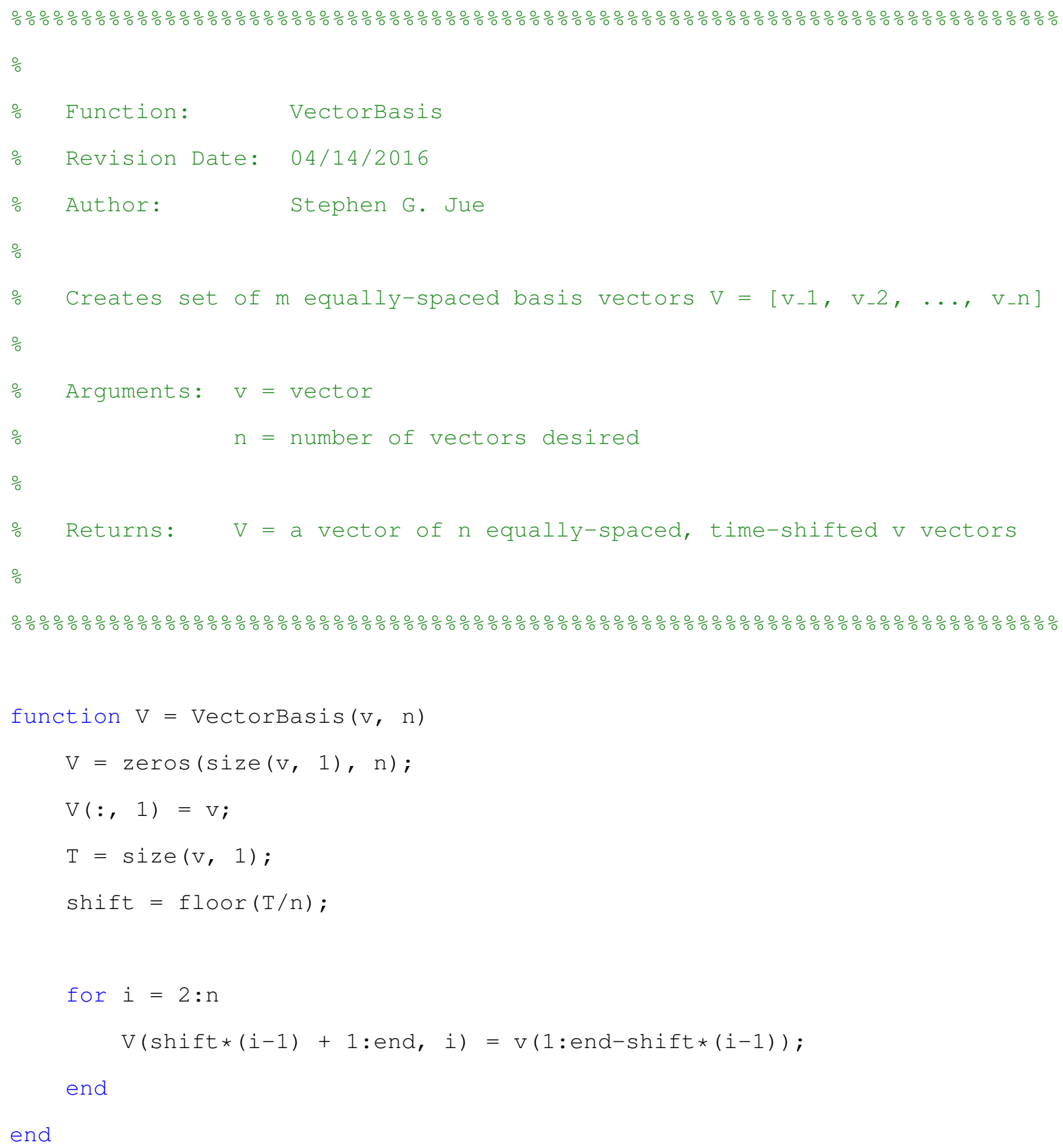




\section{A.3 PSP.m}

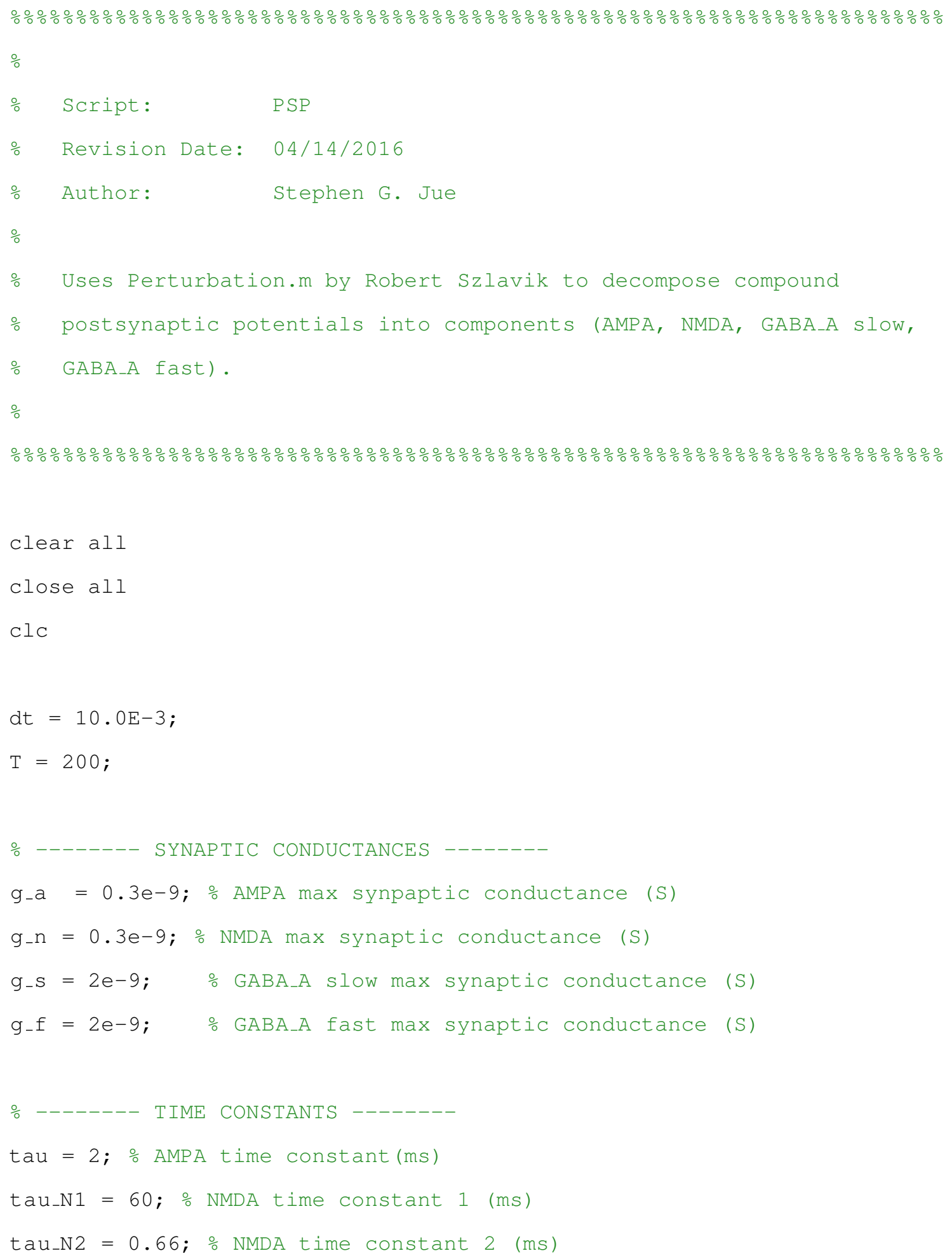




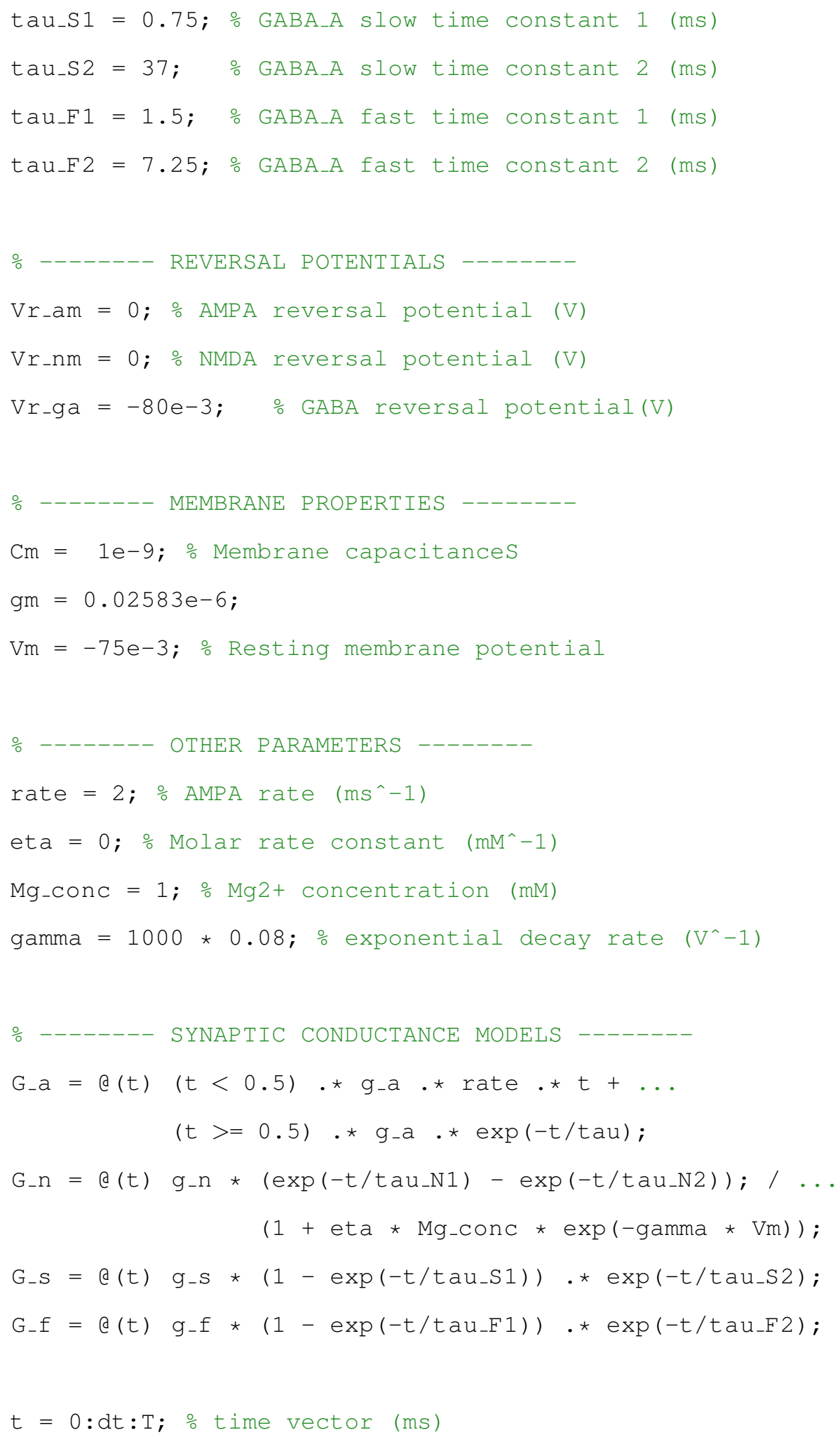




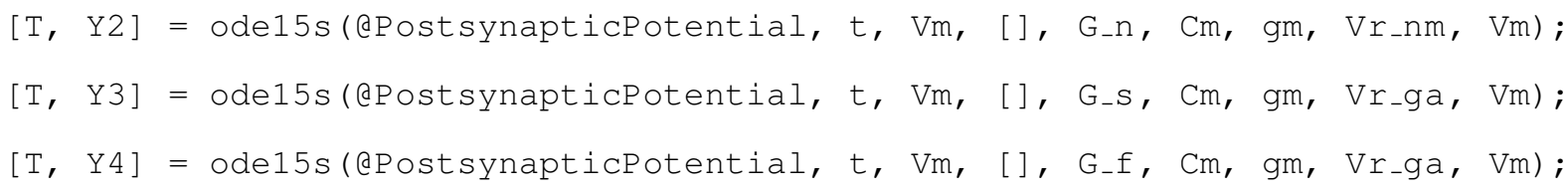




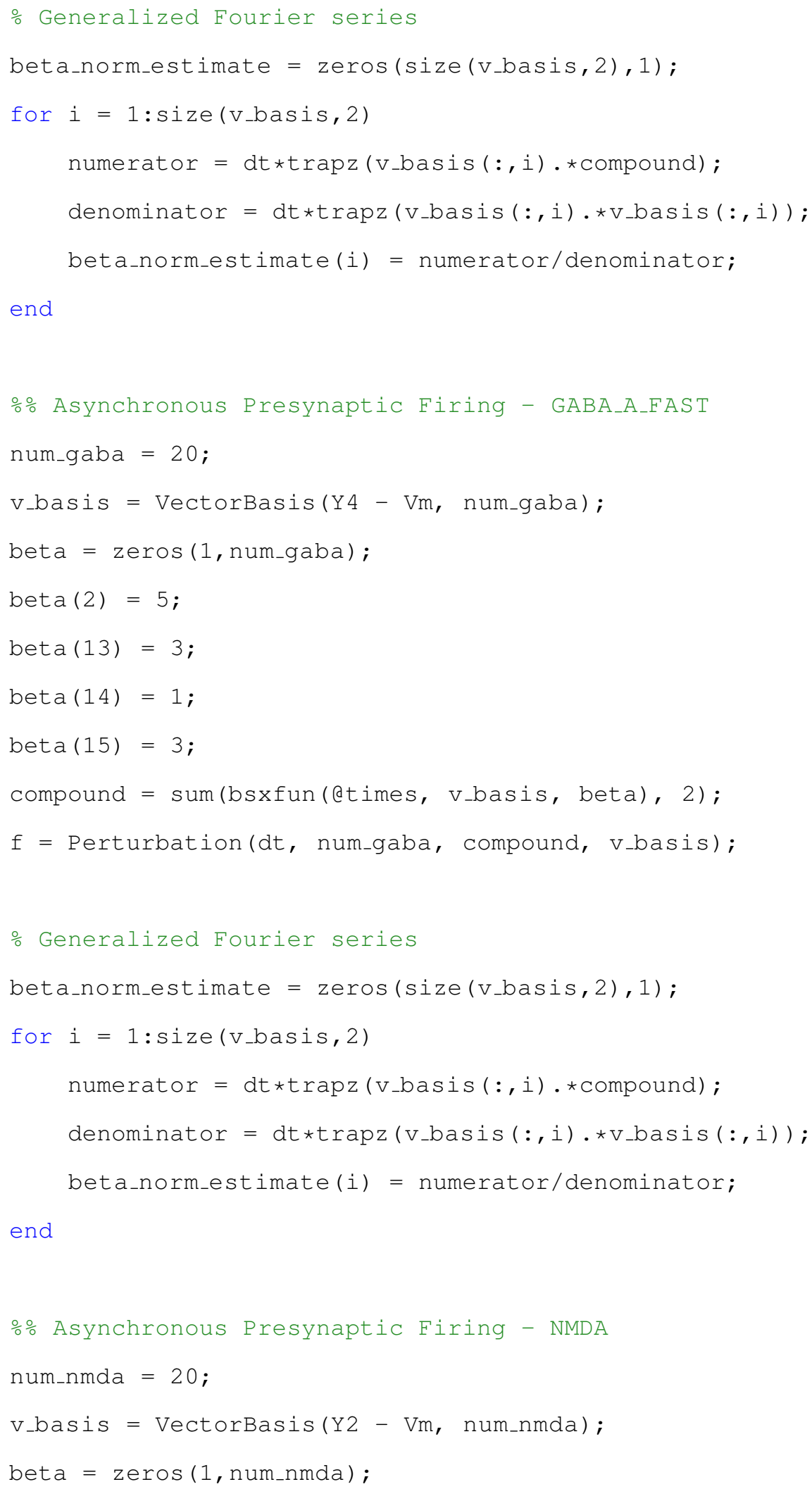




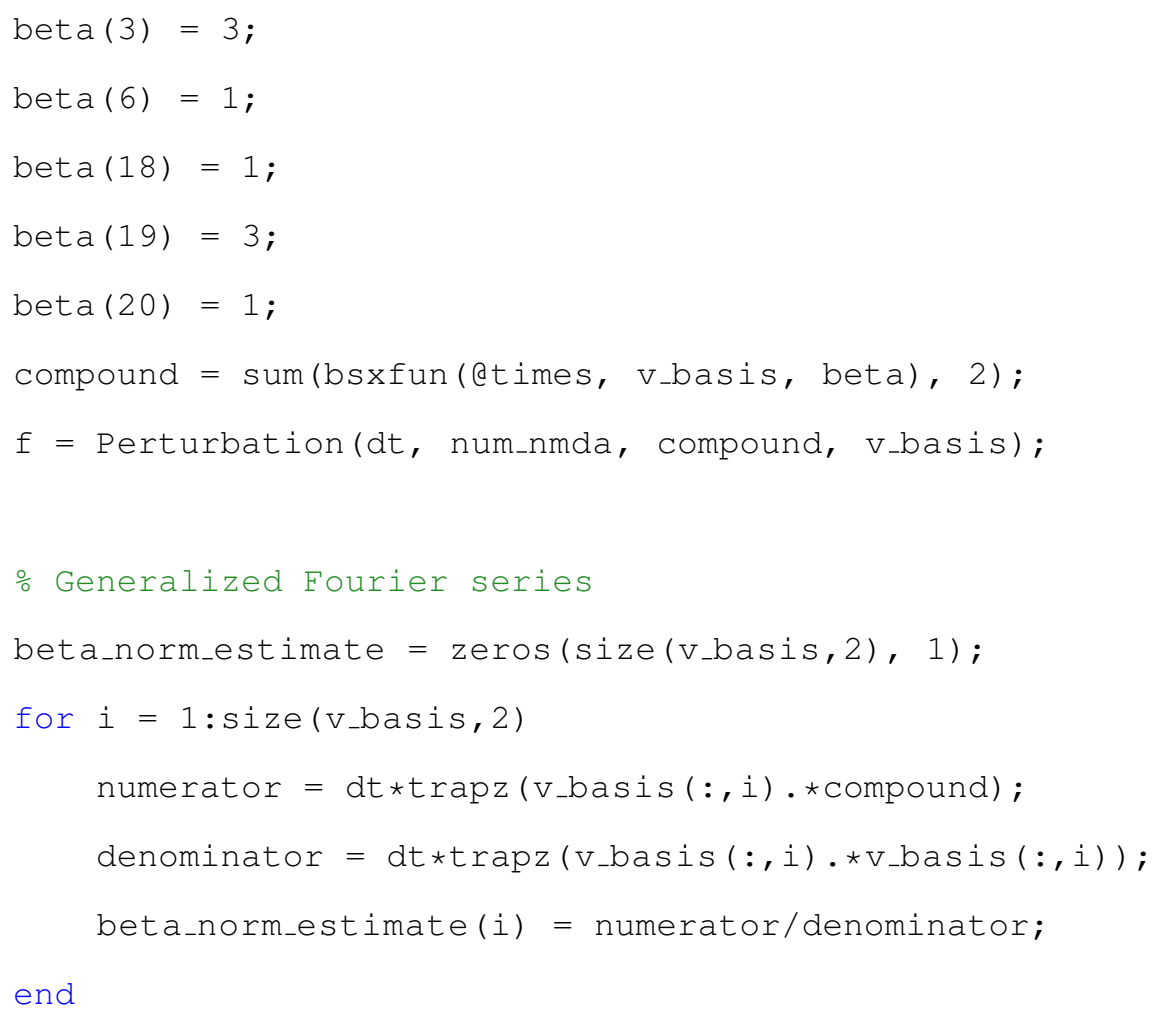




\section{A.4 PSPExperimentalValidation.m}

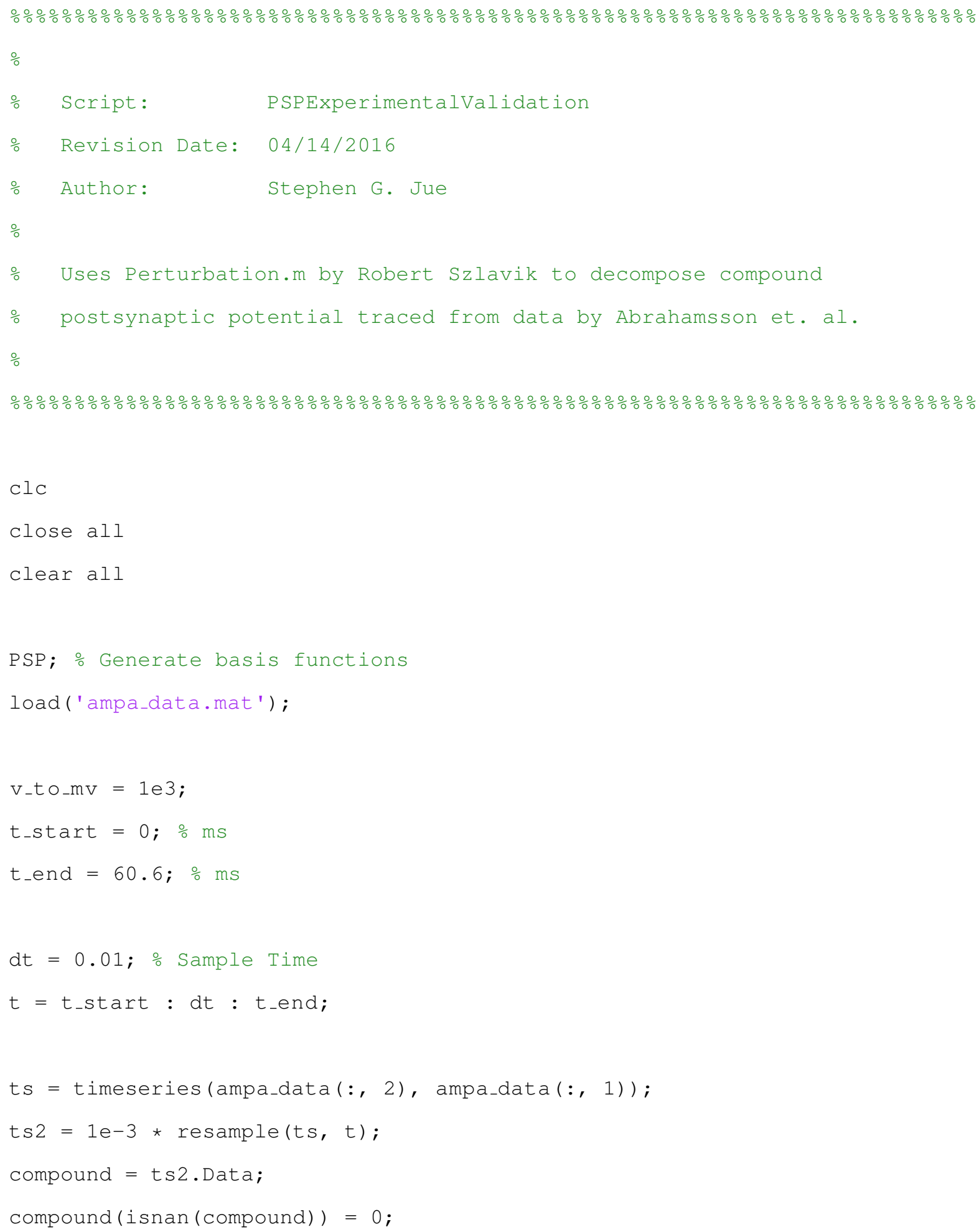




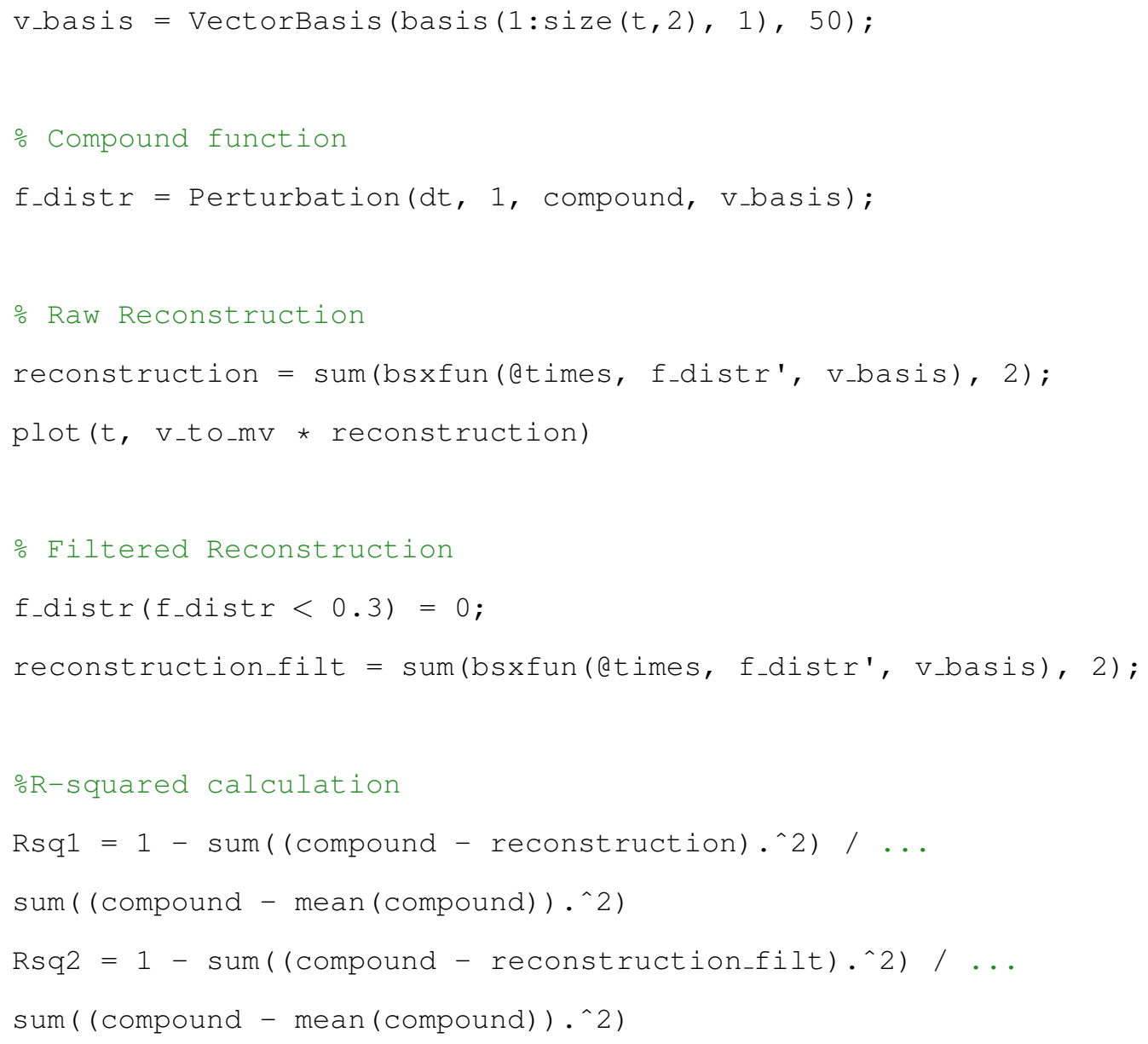

